\title{
Physical measures for infinite-modal maps
}

\author{
by \\ Vítor Araújo (Rio de Janeiro and Porto) and \\ Maria José Pacifico (Rio de Janeiro)
}

\begin{abstract}
We analyze certain parametrized families of one-dimensional maps with infinitely many critical points from the measure-theoretical point of view. We prove that such families have absolutely continuous invariant probability measures for a positive Lebesgue measure subset of parameters. Moreover, we show that both the density of such a measure and its entropy vary continuously with the parameter. In addition, we obtain exponential rate of mixing for these measures and also show that they satisfy the Central Limit Theorem.
\end{abstract}

1. Introduction. One of the main goals of dynamical systems is to describe the global asymptotic behavior of the iterates of most points under a transformation of a compact manifold, either from a topological or from a probabilistic (or ergodic) point of view. The notion of uniform hyperbolicity, introduced by Smale in [Sm], and of non-uniform hyperbolicity, introduced by Pesin $[\mathrm{P}]$, have been the main tools to rigorously establish general results in the field.

While uniform hyperbolicity is defined using only a finite number of iterates of a given transformation, non-uniform hyperbolicity is an asymptotic notion from the very beginning, demanding the existence of non-zero Lyapunov exponents almost everywhere with respect to some invariant probability measure.

On the one hand, the study of consequences of both properties in a general setting has a long history (see [M, S, KH, B, BP, Y, BDV] for details and thorough references).

On the other hand, it is rather hard in general to verify non-uniform hyperbolicity, since we must take into account the behavior of the iterates

2000 Mathematics Subject Classification: Primary 37C40; Secondary 37D25, 37A25, 37A35.

Key words and phrases: SRB measures, absolutely continuous invariant measures, infinitemodal maps, statistical stability, exponential decay of correlations, central limit theorem, continuous variation of entropy. 
of the given map when time goes to infinity. This was first achieved in the groundbreaking work of Jakobson $[\mathrm{J}]$ on the quadratic family, which was extended to more general one-dimensional families with a unique critical point by many other mathematicians (see e.g. [BC1, R, MS, T, TTY]). One-dimensional families with two critical points were first considered in [Ro], and multimodal maps and maps with critical points and singularities with unbounded derivative were treated in [LT, LV, BLS]. To the best of our knowledge, maps with infinitely many critical points were first dealt with in [PRV].

The aim of this paper is prove that the dynamics of the family considered in $[\mathrm{PRV}]$, for a positive Lebesgue measure subset of parameters, is nonuniformly hyperbolic, and to deduce some consequences from the ergodic point of view. These families naturally appear as one-dimensional models for the dynamical behavior near the unfolding of a double saddle-focus homoclinic connection of a flow in a three-dimensional manifold (see Figure 1 and $[\mathrm{Sh}]$ ). The main novelty is that we prove global stochastic behavior for a family of maps with infinitely many regions of contraction.

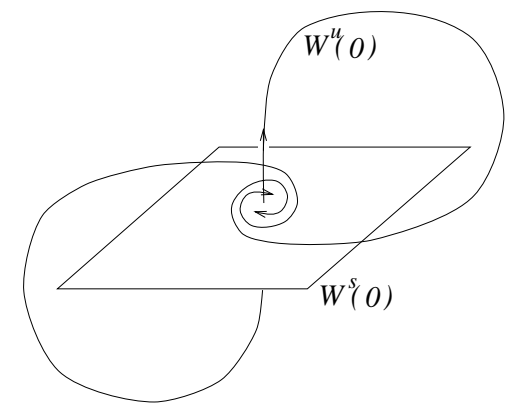

Fig. 1. Double saddle-focus homoclinic connections

Roughly speaking, the family $f_{\mu}$ of one-dimensional circle maps which we consider here is obtained from first-return maps of the three-dimensional flow in Figure 1 to appropriate cross-sections and disregarding one of the variables. This reduction to a one-dimensional model greatly simplifies the study of this kind of unfolding and provides important insight into its behavior. However, as we shall see, the dynamics of the reduced model is still highly complex.

This family of maps is obtained by translating the left hand side and right hand side, vertically in opposite directions, of the graph of the map $f=f_{0}$ described in Figure 2. This family approximates the behavior of any generic unfolding of $f_{0}$. Such unfolding was first studied in [PRV], where it was shown that for a positive Lebesgue measure subset $S$ of parameters the map $f_{\mu}$, for $\mu \in S$, exhibits a chaotic attractor. This was achieved by 
proving that the orbits of the critical values of $f_{\mu}$ have positive Lyapunov exponent and that $f_{\mu}$ has a dense orbit.

Here we complement the topological description of the dynamics of $f_{\mu}$ provided in [PRV] for $\mu \in S$ with a probabilistic description constructing for the same parameters a physical probability measure $\nu_{\mu}$. We say that an invariant probability measure $\nu$ is physical or Sinai-Ruelle-Bowen (SRB) if there is a positive Lebesgue measure set of points $x \in \mathbb{S}^{1}$ such that

$$
\lim _{n \rightarrow \infty} \frac{1}{n} \sum_{k=0}^{n-1} \varphi\left(f_{\mu}^{k}(x)\right)=\int \varphi d \nu
$$

for any observable (continuous function) $\varphi: \mathbb{S}^{1} \rightarrow \mathbb{R}$. The set of points $x \in \mathbb{S}^{1}$ with this property is called the basin of $\nu$. SRB measures provide a statistical description of the asymptotic behavior of a large subset of orbits. Combining this with the results from [PRV] we see that $f_{\mu}$ has non-zero Lyapunov exponent almost everywhere with respect to $\nu_{\mu}$, i.e. $f_{\mu}$ is nonuniformly hyperbolic for $\mu \in S$.

The main feature needed for the construction of such measures is to obtain positive Lyapunov exponent for Lebesgue almost every point under the action of $f_{\mu}, \mu \in S$. The presence of critical points is a serious obstruction to achieving an asymptotic expansion rate of the derivative at most points. Therefore the control of derivatives along orbits of the critical values is a central subject in the ergodic theory of one-dimensional maps.

The crucial role of the orbits of the critical values in the statistical description of the global dynamics of one-dimensional maps was already present in the pioneer work of Jakobson $[\mathrm{J}]$, who considered quadratic maps and obtained SRB measures for a positive Lebesgue measure subset of parameters.

This was later followed by the celebrated papers of Benedicks and Carleson [BC1, $\mathrm{BC} 2]$, where the parameter exclusion technique was used to show that, for a positive Lebesgue measure subset of parameters, the derivative along the orbit of the unique critical value has exponential growth and satisfies what is nowadays called slow recurrence to the critical point. This is enough to construct SRB measures for those parameters.

Recently, in the unimodal setting it was established that indeed the existence of SRB measures, and the exponential growth of the derivative along the orbit of the critical value, are equivalent conditions for Lebesgue almost every parameter for which there are no sinks (see [ALM, AM1, AM2]). See also [BLS] for multimodal maps.

In [PRV] the technique of exclusion of parameters was extended to deal with infinitely many critical orbits. Here we refine this technique to obtain exponential growth of the derivatives and slow recurrence to the whole 
critical set for Lebesgue almost every orbit. By [ABV] this ensures the existence of SRB measures for every parameter $\mu \in S$ (see Subsection 1.2 and Theorem A).

Moreover, we are able to control the measure of the set of points whose orbits are too close to the critical set during the first $n$ iterates, showing that its Lebesgue measure is exponential in $n$ (see Theorem $\mathrm{B}$ ). In addition, the Lebesgue measure of the set of points for which the derivative does not grow exponentially fast in the first $n$ iterates decreases exponentially fast with $n$ (see Theorem C). By recent general results in the ergodic theory of nonuniformly hyperbolic systems [ALP, G], both estimates above taken together imply exponential decay of correlations for Hölder continuous observables for $\nu_{\mu}$ and also that $\nu_{\mu}$ satisfies the Central Limit Theorem, for all $\mu \epsilon$ $S$ (see Subsection 1.3 and Corollary D). We remark that these properties are likewise satisfied by uniformly expanding maps of $\mathbb{S}^{1}$, which are the touchstone of chaotic dynamics (see e.g. $[\mathrm{B}, \mathrm{V}]$ ), in spite of the presence of infinitely many points with unbounded contraction (critical points).

Furthermore, analyzing our arguments we observe that the estimates obtained do not depend on the choice of the parameter $\mu \in S$. According to [A, AOT] this shows that the density $d \nu_{\mu} / d \lambda$ of the SRB measure $\nu_{\mu}$ with respect to Lebesgue measure and its entropy $h_{\nu_{\mu}}\left(f_{\mu}\right)$ vary continuously with $\mu \in S$ (see Subsection 1.4 and Corollary E). This type of result was recently obtained in $[\mathrm{F}]$ for quadratic maps on the set of parameters constructed in $[\mathrm{BC} 1, \mathrm{BC} 2]$ using a similar strategy. Hence statistical properties of the maps $f_{\mu}$ for $\mu \in S$ are stable under small variations of the parameter, i.e. this family is statistically stable over $S$.

We emphasize that although the general strategy for proving our results follows [BC1, BC2, PRV, F], several new difficulties had to be overcome. Indeed, unlike [BC1, BC2, PRV] where the main purpose was to obtain positive Lyapunov exponent along the orbits of critical values, here we need to obtain positive Lyapunov exponents and slow recurrence to the critical set along almost every orbit, which forces us to control the distance to the critical set for far more iterates than in [PRV]. This demands at several places a bound on the ratio between the second derivative at points near the critical set. Moreover, there are inflection points which impose extra restrictions on the arguments used in [PRV].

Furthermore, with infinitely many critical points the derivative of the smooth map we consider here is not globally bounded (unlike any smooth unimodal family, see $[\mathrm{BC} 1, \mathrm{BC} 2, \mathrm{~F}]$ ) which demanded a proof of an exponential bound for the derivative along the orbits of critical values. In order to obtain such a bound for a positive Lebesgue measure set of parameters we changed the construction presented in [PRV] by adding a new constraint in the exclusion of parameters algorithm. 
The paper is organized as follows. We first state precisely our results in Subsections 1.2 to 1.4. We sketch the proof in Section 2. In Section 3 we explain how a sequence $\left(\mathcal{P}_{n}\right)_{n \geq 0}$ of partitions of $\mathbb{S}^{1}$ whose atoms have bounded distortion under the action of $f_{\mu}^{n}$ is constructed. Basic lemmas are stated and proved in Section 4. These are used to obtain the main estimates in Section 5. In Sections 6 and 7 we use the main estimates to deduce slow recurrence to the critical set and fast expansion for most points. In Section 8 we explain how an exponential upper bound on the growth of the derivatives along critical orbits can be obtained through an extra condition imposed on the construction performed in [PRV] without loss. Finally, in Section 9 we inspect the estimates obtained during our constructions and show that they do not depend on the parameter $\mu \in S$.

1.1. Statement of the results. Let $\hat{f}$ be the interval map $\hat{f}:\left[-\varepsilon_{1}, \varepsilon_{1}\right] \rightarrow$ $[-1,1]$ given by

$$
\hat{f}(z)= \begin{cases}a z^{\alpha} \sin (\beta \log (1 / z)) & \text { if } z>0 \\ -a|z|^{\alpha} \sin (\beta \log (1 /|z|)) & \text { if } z<0\end{cases}
$$

where $a>0,0<\alpha<1, \beta>0$ and $\varepsilon_{1}>0$ (see Figure 2).

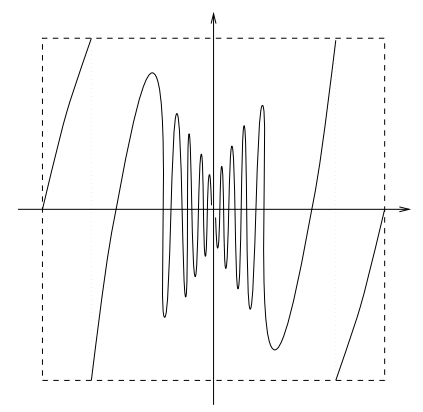

Fig. 2. Graph of the circle map $f$

Maps $\hat{f}$ as above have infinitely many critical points, of the form

$$
x_{k}=\hat{x} \exp (-k \pi / \beta) \quad \text { and } \quad x_{-k}=-x_{k} \quad \text { for each large } k>0
$$

where $\hat{x}=\exp \left(-\frac{1}{\beta} \tan ^{-1} \frac{\beta}{\alpha}\right)>0$ is independent of $k$. Let $k_{0} \geq 1$ be the smallest integer such that $x_{k}$ is defined for all $|k| \geq k_{0}$, and $x_{k_{0}}$ is a local minimum.

We extend this expression to the whole circle $\mathbb{S}^{1}=I /\{-1 \sim 1\}$, where $I=[-1,1]$, in the following way. Let $\tilde{f}$ be an orientation-preserving expanding map of $\mathbb{S}^{1}$ such that $\tilde{f}(0)=0$ and $\tilde{f}^{\prime}>\tilde{\sigma}$ for some constant $\tilde{\sigma} \gg 1$. We define $\varepsilon=2 x_{k_{0}} /\left(1+e^{-\pi / \beta}\right)$, so that $x_{k_{0}}$ is the middle point of the interval 
$\left(e^{-\pi / \beta} \varepsilon, \varepsilon\right)$, and fix two points $x_{k_{0}}<\hat{y}<\tilde{y}<\varepsilon$ with

$$
\left|\hat{f}^{\prime}(\hat{y})\right| \gg 1 \quad \text { and also } 2 \frac{1-\varepsilon^{\tau}}{1+e^{-\pi / \beta}} x_{k_{0}}>\hat{y}>x_{k_{0}},
$$

where $\tau$ is a small positive constant to be defined in what follows and we take $k_{0}=k_{0}(\tau)$ sufficiently large (and $\varepsilon$ small enough) in order that (1.3) holds. Then we take $f$ to be any smooth map on $\mathbb{S}^{1}$ coinciding with $\hat{f}$ on $[-\hat{y}, \hat{y}]$, with $\tilde{f}$ on $\mathbb{S}^{1} \backslash[-\tilde{y}, \tilde{y}]$, and monotone on each interval $\pm[\hat{y}, \tilde{y}]$.

Finally, let $f_{\mu}$ be the following one-parameter family of circle maps unfolding the dynamics of $f=f_{0}$ :

$$
f_{\mu}(z)= \begin{cases}f(z)+\mu & \text { for } z \in(0, \varepsilon] \\ f(z)-\mu & \text { for } z \in[-\varepsilon, 0)\end{cases}
$$

for $\mu \in(-\varepsilon, \varepsilon)$. For $z \in \mathbb{S}^{1} \backslash[-\varepsilon, \varepsilon]$ we assume only that $\left|\frac{\partial}{\partial z} f_{\mu}(z)\right| \geq 2$. In what follows we write $z_{k}^{ \pm}(\mu)=f_{\mu}\left(x_{k}\right)$ for $|k| \geq k_{0}$.

TheOREM 1.1 ([PRV, Theorem A]). For a given $\sigma \in(1, \sqrt{\tilde{\sigma}})$ there exists an integer $N$ such that taking $k_{0}>N$ in the construction of $\left(f_{\mu}\right)_{\mu}$, we can find a small positive constant $\tilde{\rho}$ such that for $0<\rho<\tilde{\rho}$ there exists a positive Lebesgue measure subset $S \subset\left[-\varepsilon,-\varepsilon^{2}\right] \cup\left[\varepsilon^{2}, \varepsilon\right]$ satisfying, for every $\mu \in S$,

(1) for all $n \geq 1$ and all $k_{0} \leq|k| \leq \infty$,

(a) $\left|\left(f_{\mu}^{n}\right)^{\prime}\left(z_{k}^{ \pm}(\mu)\right)\right| \geq \sigma^{n}$,

(b) either $\left|f_{\mu}^{n}\left(f_{\mu}\left(x_{l}\right)\right)\right|>\varepsilon$ or $\left|f_{\mu}^{n}\left(f_{\mu}\left(x_{l}\right)\right)-x_{m(n)}\right| \geq e^{-\rho n}$,

where $x_{m(n)}$ is the critical point nearest $f_{\mu}^{n}\left(f_{\mu}\left(x_{l}\right)\right)$;

(2) $\liminf \inf _{n \rightarrow \infty} n^{-1} \log \left|\left(f_{\mu}^{n}\right)^{\prime}(z)\right| \geq \log \sigma / 3$ for Lebesgue almost every point $z \in \mathbb{S}^{1}$

(3) there exists $z \in \mathbb{S}^{1}$ whose orbit $\left\{f_{\mu}^{n}(z): n \geq 0\right\}$ is dense in $\mathbb{S}^{1}$.

The statement of Theorem 1.1 is slightly different from the main statement of [PRV] but the proof is contained therein.

1.2. Existence of absolutely continuous invariant probability measures. The purpose of this work is to prove that for parameters $\mu \in S$ the map $f_{\mu}$ admits a unique absolutely continuous invariant probability measure $\nu_{\mu}$, whose basin covers Lebesgue almost every point of $\mathbb{S}^{1}$, and to study some of the main statistical and ergodic properties of these measures.

In what follows we write $\lambda$ for the normalized Lebesgue measure on $\mathbb{S}^{1}$. Our first result shows the existence of the SRB measure.

Theorem A. Let $\mu \in S$ be given. Then there exists an $f_{\mu}$-invariant probability measure $\nu_{\mu}$ which is absolutely continuous with respect to $\lambda$ and 
such that for $\lambda$-almost every $x \in \mathbb{S}^{1}$ and every continuous $\varphi: \mathbb{S}^{1} \rightarrow \mathbb{R}$,

$$
\lim _{n \rightarrow \infty} \frac{1}{n} \sum_{j=0}^{n-1} \varphi\left(f_{\mu}^{j}(x)\right)=\int \varphi d \nu_{\mu} .
$$

The proof is based on the technique of parameter exclusion developed in [PRV] to prove Theorem 1.1 and on recent results on hyperbolic times for non-uniformly expanding maps with singularities and criticalities, from $[\mathrm{ABV}]$.

In our setting non-uniform expansion means the same as item (2) of Theorem 1.1. However, due to the presence of (infinitely many) criticalities and the singularity at 0 , an extra condition is needed to construct the SRB measure: we need to control the average distance to the critical set along most orbits.

We say that $f_{\mu}$ has slow recurrence to the critical set $\mathcal{C}=\left\{x_{k}:|k| \geq k_{0}\right\}$ $\cup\{0\}$ if, for every $\delta>0$, there exists $b>0$ such that

$$
\limsup _{n \rightarrow \infty} \frac{1}{n} \sum_{k=0}^{n-1}-\log \operatorname{dist}_{b}\left(f_{\mu}^{k}(x), \mathcal{C}\right)<\delta
$$

for Lebesgue almost every $x \in \mathbb{S}^{1}$, where $b$ is a small positive value, and $\operatorname{dist}_{b}(x, y)=|x-y|$ if $|x-y| \leq b$ and 1 otherwise.

Let $f: I \backslash \mathcal{C} \rightarrow I$ be a $C^{2}$ map. We say that $\mathcal{C}$ is a non-flat critical set if there exist constants $B>1$ and $\beta>0$ such that

$$
\begin{aligned}
& \frac{1}{B} \operatorname{dist}(x, \mathcal{C})^{\beta} \leq\left|f^{\prime}(x)\right| \leq B \operatorname{dist}(x, \mathcal{C})^{-\beta}, \\
& |\log | f^{\prime}(x)|-\log | f^{\prime}(y)|| \leq B \frac{|x-y|}{\operatorname{dist}(x, \mathcal{C})^{\beta}},
\end{aligned}
$$

for every $x, y \in I \backslash \mathcal{C}$ with $|x-y|<\operatorname{dist}(x, \mathcal{C}) / 2$.

The following result ensuring the existence of finitely many physical probability measures is proved in $[\mathrm{ABV}]$.

THEOREM 1.2. If $f$ satisfies (S1), (S2), is non-uniformly expanding and has slow recurrence to the critical set $\mathcal{C}$, then there are finitely many ergodic absolutely continuous $f$-invariant probability measures $\mu_{1}, \ldots, \mu_{l}$ such that Lebesgue almost every point in I belongs to the basin of $\mu_{i}$ for some $i \in$ $\{1, \ldots, l\}$.

The maps $f_{\mu}$ satisfy conditions (S1)-(S2) above. Indeed, we define $y_{k}=$ $2 x_{k} /\left(1+e^{-\pi / \beta}\right)$ for each $k \geq k_{0}$, so that $x_{k}$ is the middle point of the interval $\left(y_{k+1}, y_{k}\right)$. We also use a similar notation for $k \leq-k_{0}$. We will argue using the following lemmas, which correspond to Lemmas 3.2 and 3.3 proved in $[\mathrm{PRV}]$. 
Lemma 1.3. There exists $C>0$ depending on $\hat{f}$ only (independent of $\varepsilon$ and $\mu)$ such that, for every $x \in\left(y_{l+1}, y_{l}\right)$ and $l \geq k_{0}$, respectively $x \in$ $\left(y_{l}, y_{l-1}\right)$ and $l \leq-k_{0}$, we have

(1) $C^{-1}\left|x_{l}\right|^{\alpha-2}\left|x-x_{l}\right|^{2} \leq\left|f(x)-f\left(x_{l}\right)\right| \leq C\left|x_{l}\right|^{\alpha-2}\left|x-x_{l}\right|^{2}$;

(2) $C^{-1}\left|x_{l}\right|^{\alpha-2}\left|x-x_{l}\right| \leq\left|f^{\prime}(x)\right| \leq C\left|x_{l}\right|^{\alpha-2}\left|x-x_{l}\right|$.

Lemma 1.4. Let $s, t \in\left[y_{l+1}, y_{l}\right]$ with $l \geq k_{0}$, respectively, $s, t \in\left[y_{l}, y_{l-1}\right]$ with $l \leq-k_{0}$. Then

$$
\left|\frac{f_{\mu}^{\prime}(s)-f_{\mu}^{\prime}(t)}{f_{\mu}^{\prime}(t)}\right| \leq K_{1} \frac{|s-t|}{\left|t-x_{l}\right|}
$$

where $K_{1}>0$ is independent of $l, s, t, \varepsilon$ and $\mu$.

On the one hand, since $0<\alpha<1, x \in\left(y_{l+1}, y_{l}\right)$ and $\left|x_{l}\right|<1$, from Lemma 1.3(2) we have

$$
\begin{aligned}
C\left|x_{l}\right|^{\alpha-2}\left|x-x_{l}\right| & =\left(C\left|x_{l}\right|^{\alpha-2}\left|x-x_{l}\right|^{2}\right)\left|x-x_{l}\right|^{-1} \leq\left(C\left|x_{l}\right|^{\alpha-2}\left|x_{l}\right|^{2}\right)\left|x-x_{l}\right|^{-1} \\
& \leq C\left|x-x_{l}\right|^{-1} .
\end{aligned}
$$

On the other hand, since $\alpha-2<0$ and $\left|x_{l}\right|<1$ we get $C^{-1}\left|x_{l}\right|^{\alpha-2}\left|x-x_{l}\right|$ $\geq C^{-1}\left|x-x_{l}\right|$, showing that (S1) holds for $f_{\mu}$ with $B=C$ and $\beta=1$, whenever $x \in\left(y_{k+1}, y_{k}\right)$ and $x_{k}$ is the closest critical point to $x$. Otherwise, if $x \in\left(y_{k+1}, y_{k}\right)$ and $x_{k+1}$ is the closest critical point to $x$, then we have $\left|x-x_{k}\right|>\left|x-x_{k+1}\right|$ and so by the above calculations we get

$$
\begin{aligned}
& \left|f_{\mu}^{\prime}(x)\right| \leq C\left|x-x_{k}\right|^{-1}=C\left|x-x_{k+1}\right|^{-1}\left|\frac{x-x_{k}}{x-x_{k+1}}\right|^{-1} \leq C\left|x-x_{k+1}\right|^{-1}, \\
& \left|f_{\mu}^{\prime}(x)\right| \geq \frac{1}{C}\left|x-x_{k}\right|=\frac{1}{C}\left|x-x_{k+1}\right|\left|\frac{x-x_{k}}{x-x_{k+1}}\right| \geq \frac{1}{C}\left|x-x_{k+1}\right| .
\end{aligned}
$$

This shows that (S1) is true for $f$ in all cases.

To check that (S2) also holds we write

$$
\frac{\left|f_{\mu}^{\prime}(x)\right|}{\left|f_{\mu}^{\prime}(y)\right|}=\frac{\left|f_{\mu}^{\prime}(x)-f_{\mu}^{\prime}(y)+f_{\mu}^{\prime}(y)\right|}{\left|f_{\mu}^{\prime}(y)\right|} \leq 1+\frac{\left|f_{\mu}^{\prime}(x)-f_{\mu}^{\prime}(y)\right|}{\left|f_{\mu}^{\prime}(y)\right|}
$$

and hence because $\log (1+z) \leq z$ for $z>-1$ we get

$$
|\log | f_{\mu}^{\prime}(x)|-\log | f_{\mu}^{\prime}(y)|| \leq \frac{\left|f_{\mu}^{\prime}(x)-f_{\mu}^{\prime}(y)\right|}{\left|f_{\mu}^{\prime}(y)\right|} \leq K_{1} \frac{|x-y|}{\left|x-x_{l}\right|},
$$

which, by the same observation during the proof of (S1), is enough to prove (S2) in all cases.

Thus according to Theorems 1.2 and 1.1 , we only need to show that $f_{\mu}$ has slow recurrence to the critical set for $\mu \in S$ to achieve the result stated in Theorem A. This is done in Sections 4 to 6, where a stronger result is obtained, as explained in what follows. 
1.3. Exponential decay of correlations and Central Limit Theorem. Using some recent developments on the statistical behavior of non-uniformly expanding maps [ALP, G] we are able to obtain exponential bounds on the decay of correlations between Hölder continuous observables for $\nu_{\mu}$ with $\mu \in S$. In addition, it follows from standard techniques that $\nu_{\mu}$ also satisfies the Central Limit Theorem. In order to achieve this we refine the arguments in [PRV] using strong conditions on the exclusion of parameters extending the estimates obtained therein for critical orbits to get an exponential upper bound on the growth of the derivative along orbits of critical values, as explained in Section 8. Moreover, we are able to extend most of the estimates from [PRV] for Lebesgue almost every orbit, yielding an exponential bound on the Lebesgue measure of the set of points whose average distance to the critical set during the first $n$ iterates is small, as follows.

We first define the average distance to the critical set

$$
\mathcal{C}_{n}^{b}(x)=\frac{1}{n} \sum_{j=0}^{n-1}-\log \operatorname{dist}_{b}\left(f_{\mu}^{j}(x), \mathcal{C}\right) .
$$

for a given $b>0$. Then we are able to prove the following.

Theorem B. Let $\mu \in S$ and $\delta>0$ be given. Then there are constants $C_{1}, \xi_{1}, b>0$ depending on $\hat{f}, \sigma, k_{0}$ and $\delta$ only such that $\mathcal{R}(x)=\min \{N \geq 1$ : $\left.\mathcal{C}_{n}^{b}(x)<\delta, \forall n \geq N\right\}$ satisfies

$$
\lambda\left(\left\{x \in \mathbb{S}^{1}: \mathcal{R}(x)>n\right\}\right) \leq C_{1} e^{-\xi_{1} n} .
$$

We note that in particular this shows that $f_{\mu}$ has slow recurrence to the critical set and ensures the existence of the SRB measure $\nu_{\mu}$ for $\mu \in S$ by Theorem 1.2.

We are also able to obtain, using the same techniques, an exponential bound on the set of points whose expansion rate up to time $n$ is less than the one prescribed by item (2) of Theorem 1.1. This is detailed in Section 7.

Theorem C. Let $\mu \in S$ be given. Then there exist constants $C_{2}, \xi_{2}>0$ depending on $\hat{f}, \rho$ and $k_{0}$ only such that $\mathcal{E}(x)=\min \left\{N \geq 1:\left|\left(f_{\mu}^{n}\right)^{\prime}(x)\right|>\right.$ $\left.\sigma^{n / 3}, \forall n \geq N\right\}$ satisfies

$$
\lambda\left(\left\{x \in \mathbb{S}^{1}: \mathcal{E}(x)>n\right\}\right) \leq C_{2} e^{-\xi_{2} n} .
$$

In particular we obtain a new proof of item (2) of Theorem 1.1, which does not follow directly from Theorem A plus the Ergodic Theorem since it is not obvious whether $\log \left|f^{\prime}\right|$ is $\nu_{\mu}$-integrable.

Theorems B and $\mathrm{C}$ ensure that for $\mu \in S$ there are constants $C_{3}>0$ and $\xi_{3} \in(0,1)$ such that $\Gamma_{n}=\left\{x \in \mathbb{S}^{1}: \mathcal{E}(x)>n\right.$ or $\left.\mathcal{R}(x)>n\right\}$ satisfies

$$
\lambda\left(\Gamma_{n}\right) \leq C_{3} e^{-\xi_{3} n}
$$

for all $n \geq 1$. This fits nicely into the following statements. 
TheOREM 1.5. Let $g: \mathbb{S}^{1} \rightarrow \mathbb{S}^{1}$ be a transitive $C^{2}$ local diffeomorphism outside a non-flat critical set $\mathcal{C}$ such that (1.8) holds. Then

(1) ([ALP, Theorem 1]) there exists an absolutely continuous invariant probability measure $\nu$ and some finite power of $g$ is mixing with respect to $\nu$

(2) ([G, Theorem 1.1]) there exist constants $C, c>0$ such that the correlation function $\operatorname{Corr}_{n}(\varphi, \psi)=\left|\int\left(\varphi \circ g^{n}\right) \psi d \nu-\int \varphi d \nu \int \psi d \nu\right|$, for Hölder continuous observables $\varphi, \psi: \mathbb{S}^{1} \rightarrow \mathbb{R}$, satisfies, for all $n \geq 1$,

$$
\operatorname{Corr}_{n}(\varphi, \psi) \leq C e^{-c n} .
$$

(3) ([ALP, Theorem 4]) $\nu$ satisfies the Central Limit Theorem: given a Hölder continuous function $\phi: \mathbb{S}^{1} \rightarrow \mathbb{R}$ which is not a coboundary $\left(\phi \neq \psi \circ g-\psi\right.$ for any $\left.\psi: \mathbb{S}^{1} \rightarrow \mathbb{R}\right)$ there exists $\theta>0$ such that for every interval $J \subset \mathbb{R}$,

$$
\begin{aligned}
\lim _{n \rightarrow \infty} \nu\left(\left\{x \in \mathbb{S}^{1}: \frac{1}{\sqrt{n}} \sum_{j=0}^{n-1}\left(\phi\left(g^{j}(x)\right)-\int \phi d \nu\right)\right.\right. & \in J\}) \\
& =\frac{1}{\theta \sqrt{2 \pi}} \int_{J} e^{-t^{2} /\left(2 \theta^{2}\right)} d t .
\end{aligned}
$$

It is then straightforward to deduce the following conclusion.

Corollary D. For every $\mu \in S$ the map $f_{\mu}$ has exponential decay of correlations for Hölder continuous observables and satisfies the Central Limit Theorem with respect to the SRB measure $\nu_{\mu}$.

1.4. Continuous variation of densities and of entropy. We note that during the arguments in Sections 2 to 7 the constants used in every estimation depend uniformly on the values of $\rho, \sigma$ and $\varepsilon$ which can be set right from the start of the construction that proves Theorems B and C. This enables us to use recent results of statistical stability and continuity of the SRB entropy from [A, AOT], showing that both the densities of the $S R B$ measures $\nu_{\mu}$ and the entropy vary continuously with $\mu \in S$.

Let $\mathcal{F}$ be a family of $C^{2}$ maps of $\mathbb{S}^{1}$ outside a fixed non-flat critical set $\mathcal{C}$ such that for any given $f \in \mathcal{F}$ and $\delta_{1}>0$ there exists $\delta_{2}>0$ satisfying, for every measurable subset $E \subset \mathbb{S}^{1}$,

$$
\lambda(E)<\delta_{2} \Rightarrow \lambda\left(f^{-1}(E)\right)<\delta_{1},
$$

that is, $f_{*}(\lambda) \ll \lambda$. We say that a family $\mathcal{F}$ as above is a non-degenerate family of maps. 
TheOREM 1.6. Let a non-degenerate family $\mathcal{F}$ of $C^{2}$ maps of $\mathbb{S}^{1}$ outside a fixed non-flat critical set $\mathcal{C}$ be given such that for every $f \in \mathcal{F}$ the corresponding functions $\mathcal{E}, \mathcal{R}: \mathbb{S}^{1} \rightarrow \mathbb{N}$ define a family $\left(\Gamma_{n}\right)_{n \geq 1}$ satisfying (1.8) with constants $C_{3}, \xi_{3}$ not depending on $f \in \mathcal{F}$. Then

(1) $\left(\left[\mathrm{A}\right.\right.$, Theorem A]) the map $\left(\mathcal{F}, d_{C^{2}}\right) \rightarrow\left(L^{1}(\lambda),\|\cdot\|_{1}\right), f \mapsto d \nu_{f} / d \lambda \in$ $L^{1}(\lambda)$, is continuous, where $d_{C^{2}}$ is the $C^{2}$ distance and $\|\cdot\|_{1}$ the $L^{1}$-norm;

(2) ([AOT, Corollary C]) the map $\left(\mathcal{F}, d_{C_{2}}\right) \rightarrow \mathbb{R}, f \mapsto h_{\nu_{f}}(f)$, is continuous.

We observe that $\mathcal{F}=\left\{f_{\mu}: \mu \in S\right\}$ satisfies all the above conditions since

- $\hat{f}$ is a $C^{\infty}$ map whose non-zero singularities, albeit infinitely many, are of quadratic type, and near zero $\hat{f}$ is bounded by $|z|^{\alpha}$;

- $f_{\mu}$ is obtained from $\hat{f}$ through a local diffeomorphism extension plus two translations (or rigid rotations when viewed on $\mathbb{S}^{1}$ );

- the values of $\beta, \varepsilon, \sigma, \rho$ can be chosen so that

- $S$ is given by Theorem 1.1 with positive Lebesgue measure;

- $f_{\mu}$ for $\mu \in S$ satisfies (1.8) with $C_{3}, \xi_{3}>0$ depending only on $\varepsilon, \sigma, \rho$ this is detailed in Section 9.

Thus we deduce the following corollary which shows that statistical properties of $f_{\mu}$ are stable under small variations of the parameter $\mu$ within the set $S$.

COROllary E. The following maps are both continuous:

$$
\begin{aligned}
S & \rightarrow\left(L^{1}(\lambda),\|\cdot\|_{1}\right), & & S \rightarrow \mathbb{R}, \\
\mu & \mapsto \frac{d \nu_{\mu}}{d \lambda}, & \text { and } & \mu \mapsto h_{\nu_{\mu}}(f) .
\end{aligned}
$$

2. Idea of the proof. From now on we fix a parameter $\mu \in S$ and write $\mathcal{C}_{\infty}=\bigcup_{n=0}^{\infty}\left(f^{n}\right)^{-1}(\mathcal{C})$ for the set of pre-orbits of the critical set $\mathcal{C}$. We also write $f=f_{\mu}$ in what follows.

Following [PRV] we consider a convenient partition $\{I(l, s, j)\}$ of the phase space into subintervals, with a bounded distortion property: trajectories with the same itinerary with respect to this partition have derivatives which are comparable, up to a multiplicative constant. This is done as follows. Let $l \geq k_{0}$ and $y_{l} \in\left(x_{l}, x_{l-1}\right)$ be as defined in Subsections 1.1 and 1.2: $x_{l}$ is the middle point of $\left(y_{l+1}, y_{l}\right)$. We partition $\left(x_{l}, y_{l}\right)$ into subintervals $I(l, s)=\left(x_{l}+e^{-(\pi / \beta) s}\left(y_{l}-x_{l}\right), x_{l}+e^{-(\pi / \beta)(s-1)}\left(y_{l}-x_{l}\right)\right), s \geq 1$. We denote by $I(l,-s)$ the subinterval of $\left(y_{l+1}, x_{l}\right)$ symmetrical to $I(l, s)$ with respect to $x_{l}$. We subdivide $I(l, \pm s)$ into $(l+|s|)^{3}$ intervals $I(l, \pm s, j), 1 \leq j \leq(l+|s|)^{3}$, 


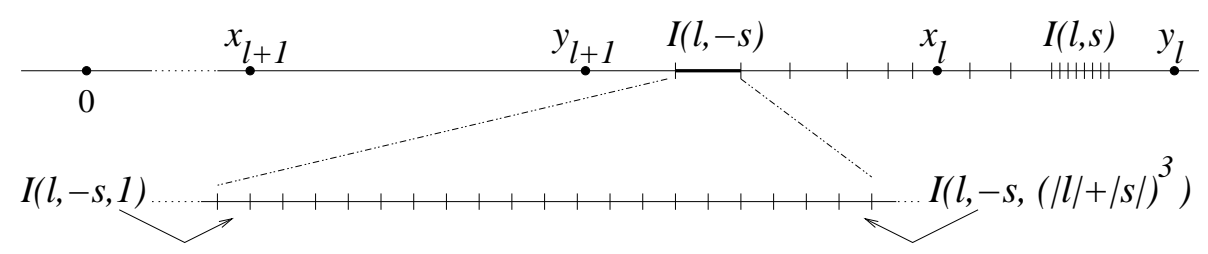

Fig. 3. The initial partition $\mathcal{P}_{0}$

with equal length and $j$ increasing as $I(l, \pm s, j)$ is closer to $x_{l}$ (see Figure 3 ). We also perform entirely symmetric constructions for $l \leq-k_{0}$. Let $I\left( \pm k_{0}, 1,1\right)$ be the intervals having $\pm \varepsilon$ in their boundaries. Clearly we may suppose that $I\left( \pm k_{0}, 1,1\right)$ are contained in the region $\mathbb{S}^{1} \backslash[-\tilde{y}, \tilde{y}]$ where $f$ coincides with $\tilde{f}$, and so $\left|f^{\prime}\right|>\sigma_{0}>1$. Finally, for completeness, we set $I(0,0,0)=I(0,0)=\mathbb{S}^{1} \backslash[-\varepsilon, \varepsilon]$.

REMARK 2.1. By the definition of $I(l, s, j)$,

$$
|I(l, s, j)|=a_{1} \frac{e^{-(\pi / \beta)(|l|+|s|)}}{(|l|+|s|)^{3}}
$$

and

$$
a_{2} e^{-(\pi / \beta)(|l|+|s|)} \leq \operatorname{dist}\left(I(l, s, j), x_{l}\right) \leq a_{2} e^{-(\pi / \beta)(|l|+|s|-1)}
$$

where $|I|$ denotes the length of the interval $I$,

$$
a_{1}=\hat{x} \frac{\left(e^{\pi / \beta}-1\right)^{2}}{e^{\pi / \beta}+1} \text { and } a_{2}=\hat{x} \frac{e^{\pi / \beta}-1}{e^{\pi / \beta}+1}<1 .
$$

Moreover, for any $m \geq 1$ we have $\left|x_{m}-x_{m+1}\right|=\hat{x}\left(1-e^{-\pi / \beta}\right) e^{-(\pi / \beta) m}$. In addition, we have $\operatorname{dist}(I(l, s, j), 0)=\left|x_{l}\right| \pm \operatorname{dist}\left(I(l, s, j), x_{l}\right)$ according to the sign of $s$ and consequently $\left|\hat{x}-a_{2}\right| e^{-(\pi / \beta)|l|} \leq \operatorname{dist}(I(l, s, j), 0) \leq$ $\left(a_{2}+\hat{x}\right) e^{-(\pi / \beta)|l|}$.

We will separate the orbit of a point $x_{0} \in I \backslash \mathcal{C}_{\infty}$ into sequences of consecutive iterates according to whether the point is near $\mathcal{C}$ or is in the expanding region $I(0,0,0)$. When $x_{n}=f^{n}\left(x_{0}\right)$ is near $\mathcal{C}$, we say that $n$ is a return time and the expansion may be lost. But since we know that for $\mu \in S$ the derivatives along the critical orbits grow exponentially fast, we shadow the orbit of $x_{n}$ during a binding period by the orbit of the nearest critical point and borrow its expansion. At the end of this binding period, the expansion is completely recovered, which will be explained precisely in Section 4.

This picture is complicated by the infinite number of critical points and by the possible returns near another critical point during a binding period. Iterates outside binding periods and return times are free iterates, where the derivative is uniformly expanded. 
Our main objective is to obtain slow recurrence to $\mathcal{C}$, which means that the returns of generic orbits are not too close to $\mathcal{C}$ on the average. However, even at a free iterate the orbits may be very close to the critical set, by the geometry of the graph of $f_{0}$, which demands a deeper analysis to achieve slow recurrence to the critical set. Moreover, since $\left|f^{\prime}\right|$ is not bounded from above in our setting, we do not automatically have an exponential bound on the derivative along orbits of critical values, which is needed to better control the recurrence to $\mathcal{C}$ and must be proved by a separate argument involving a stronger exclusion of parameters than in the algorithm presented in [PRV].

Using the slow recurrence we show that the derivative along the orbit of Lebesgue almost every point grows exponentially fast. Using the estimates from Sections 3 to 5 we are able to obtain more: we deduce the exponential estimates of Theorems B and C in Sections 6 and 7 .

Finally, the refinement of the parameter exclusion in [PRV] and the dependence of the constants on the choices made during the entire construction are detailed in Sections 8 and 9 respectively, where we show that the estimates are uniform in $\mu \in S$.

3. Refining the partition. We are going to build inductively a sequence of partitions $\mathcal{P}_{0}, \mathcal{P}_{1}, \ldots$ of $I$ (modulo a zero Lebesgue measure set) into intervals. We will define inductively the set $R_{n}(\omega)=\left\{r_{1}, \ldots, r_{\gamma(n)}\right\}$, which is the set of return times of $\omega \in \mathcal{P}_{n}$ up to $n$, and a set $Q_{n}(\omega)=$ $\left\{\left(l_{1}, s_{1}, j_{1}\right), \ldots,\left(l_{\gamma(n)}, s_{\gamma(n)}, j_{\gamma(n)}\right)\right\}$, which records the indices of the intervals such that $f^{r_{i}}(\omega) \subset I\left(l_{i}, s_{i}, j_{i}\right), i=1, \ldots, r_{\gamma(n)}$.

In the process we will show inductively that for all $n \in \mathbb{N}_{0}$,

$$
\left.\forall \omega \in \mathcal{P}_{n} \quad f^{n+1}\right|_{\omega} \text { is a diffeomorphism, }
$$

which is essential for the construction itself. For $n=0$ we define

$$
\mathcal{P}_{0}=\{I(0,0,0)\} \cup\left\{I(l, s, j):|l| \geq k_{0},|s| \geq 1,1 \leq j \leq(|l|+|s|)^{3}\right\} .
$$

It is obvious that $\mathcal{P}_{0}$ satisfies (3.1) for $n=0$. We set $R_{0}(I(0,0,0))=\emptyset$ and $R_{0}(I(l, s, j))=\{0\}, Q_{0}(I(l, s, j))=\{(l, s, j)\}$ for all possible $(l, s, j) \neq$ $(0,0,0)$.

REMARK 3.1. This means that every $I(l, s, j)$ with $|l| \geq k_{0},|s| \geq 1$ and $j=1, \ldots,(|l|+|s|)^{3}$ has a return at time 0 , by definition. This will be important in Section 6.

For each $(l, s)$ with $|l| \geq k_{0}$ and $|s| \geq 1$ such that

$$
e^{-(\pi / \beta)|s|} \frac{1-e^{-\pi / \beta}}{1+e^{-\pi / \beta}}<\tau, \quad \text { i.e. }|s|>s(\tau)=-\frac{\beta}{\pi} \log \left(\tau \frac{1+e^{-\pi / \beta}}{1-e^{-\pi / \beta}}\right),
$$


we define the binding period $p(x)$ of $x \in I(l, s)$ to be the largest integer $p>0$ such that

$$
\left|f^{h}\left(x_{l}\right)\right| \leq \varepsilon \quad \text { and } \quad\left|f^{h}(x)-f^{h}\left(x_{l}\right)\right| \leq\left|f^{h}\left(x_{l}\right)-x_{m(h-1)}\right| e^{-\tau h}
$$

or

$\left|f^{h}\left(x_{l}\right)\right|>\varepsilon \quad$ and $\quad\left|f^{h}(x)-f^{h}\left(x_{l}\right)\right| \leq \varepsilon^{1+\tau} e^{-\tau h}$

for all $1 \leq h \leq p$, where $x_{m(h)}$ is the critical point nearest $f^{h}\left(f\left(x_{l}\right)\right)$ and $\tau>0$ is a small constant to be specified during the construction.

Condition (3.2) failing means that $I(l, s)$ is not close enough to $\mathcal{C}$ since

$$
\left|x-x_{l}\right| \geq e^{-(\pi / \beta)|s|}\left(y_{l}-x_{l}\right) \geq e^{-(\pi / \beta)|s|} \frac{1-e^{-\pi / \beta}}{1+e^{-\pi / \beta}}\left|x_{l}\right| \geq \tau\left|x_{l}\right|
$$

for all $x \in I(l, s)$, and in this case there is no expansion loss at the point $x$. Indeed, by Lemma 1.3 and using the definition of $x_{l}$ from (1.2) we get

$$
\left|f^{\prime}(x)\right| \geq C^{-1}\left|x_{l}\right|^{\alpha-2}\left|x-x_{l}\right| \geq C^{-1}\left|x_{l}\right|^{\alpha-2} \tau\left|x_{l}\right|=\frac{\tau \hat{x}^{\alpha-1}}{C} e^{(1-\alpha)(\pi / \beta)|l|} .
$$

Since $1-\alpha>0$ and $|l| \geq k_{0}$, this ensures that $\left|f^{\prime}(x)\right|>1$ if we take $k_{0}=k_{0}(\tau)$ large enough.

REMARK 3.2. As we will explain along the proof, the values of $k_{0}$ and $\tau^{-1}$ will both need to be taken sufficiently large. We note that $k_{0} \rightarrow \infty$ as $\tau \rightarrow 0^{+}$. For more on these dependencies see Section 9 .

We define the binding period $p(l, s)$ of the interval $I(l, s)$ to be the smallest binding period of all points of this interval, that is, $p(l, s)=\inf \{p(x)$ : $x \in I(l, s)\}$.

For $(l, s, j)$ with $|l| \geq k_{0},|s| \geq 1$ and $1 \leq j \leq(|l|+|s|)^{3}$, write $I(l, s, j)^{+}$ for the union of $I(l, s, j)$ with its two adjacent intervals in $\mathcal{P}_{0}$.

Now we assume that $\mathcal{P}_{n-1}$ is defined and satisfies (3.1), and $R_{n-1}, Q_{n-1}$ are also defined on each element of $\mathcal{P}_{n-1}$. For a fixed interval $\omega \in \mathcal{P}_{n-1}$ there are three possible situations.

(1) If $R_{n-1}(\omega) \neq \emptyset$ and $n<r_{\gamma(n-1)}+p\left(l_{\gamma(n-1)}, s_{\gamma(n-1)}\right)$ then we say that $n$ is a bound time for $\omega$, put $\omega \in \mathcal{P}_{n}$ and set $R_{n}(\omega)=R_{n-1}(\omega)$, $Q_{n}(\omega)=Q_{n-1}(\omega)$.

(2) If either $R_{n-1}(\omega)=\emptyset$, or $n \geq r_{\gamma(n-1)}+p\left(l_{\gamma(n-1)}, s_{\gamma(n-1)}\right)$ and $f^{n}(\omega) \subset I(0,0,0) \cup I\left( \pm k_{0}, 1,1\right)$ and $f^{n}(\omega)$ does not contain any $I\left( \pm k_{0}, 1,1\right)$, then we say that $n$ is a free time for $\omega$, put $\omega \in \mathcal{P}_{n}$ and set $R_{n}(\omega)=R_{n-1}(\omega), Q_{n}(\omega)=Q_{n-1}(\omega)$.

(3) If the two conditions above fail then we consider two cases:

(a) $f^{n}(\omega)$ does not cover completely any $I(l, s, j)$, with $|l| \geq k_{0}$, $|s| \geq 1$ and $j=1, \ldots,(|l|+|s|)^{3}$. Because $f^{n}$ is continuous 
and $\omega$ is an interval, $f^{n}(\omega)$ is also an interval and thus it is contained in some $I(l, s, j)^{+}$, for certain $|l| \geq k_{0},|s| \geq 1$ and $l=1, \ldots,(|l|+|s|)^{3}$, which is called the host interval.

If $|s|>s(\tau)$, then this $n$ is an inessential return time for $\omega$ and we set $R_{n}(\omega)=R_{n-1}(\omega) \cup\{n\}, Q_{n}(\omega)=Q_{n-1}(\omega) \cup\{(l, s, j)\}$ and put $\omega \in \mathcal{P}_{n}$.

Otherwise, $|s| \leq s(\tau)$ and there is no expansion loss, thus $n$ is again a free time, put $\omega \in \mathcal{P}_{n}$ and set $R_{n}(\omega)=R_{n-1}(\omega)$, $Q_{n}(\omega)=Q_{n-1}(\omega)$

(b) $f^{n}(\omega)$ contains at least one interval $I(l, s, j)$ with $|l| \geq k_{0},|s| \geq 1$ and $j=1, \ldots,(|l|+|s|)^{3}$, in which case we partition $\omega$ as follows. We consider the sets

$$
\begin{aligned}
& \omega_{l, s, j}^{\prime}=f^{-n}(I(l, s, j)) \cap \omega \quad \text { for }|l| \geq k_{0},|s| \geq 1, \\
& \quad 1 \leq j \leq(|l|+|s|)^{3} \text { and }(|l|, s, j) \neq\left(k_{0}, 1,1\right), \\
& \omega_{0,0,0}^{\prime}=f^{-n}\left(I(0,0,0) \cup I\left( \pm k_{0}, 1,1\right)\right) \cap \omega .
\end{aligned}
$$

Denoting by $\mathcal{I}$ the set of indices $(l, s, j)$ such that $\omega_{l, s, j}^{\prime} \neq \emptyset$ we have

$$
\omega \backslash f^{-n}(\mathcal{C})=\bigcup_{(l, s, j) \in \mathcal{I}} \omega_{l, s, j}^{\prime} .
$$

By the induction hypothesis $\left.f^{n}\right|_{\omega}$ is a diffeomorphism and then each $\omega_{l, s, j}^{\prime}$ is an interval. Moreover, $f^{n}\left(\omega_{l, s, j}^{\prime}\right)$ covers the whole $I(l, s, j)$ for $|l| \geq k_{0},|s| \geq 1, j=1, \ldots,(|l|+|s|)^{3}$, except possibly for one or two end intervals. When $f^{n}\left(\omega_{l, s, j}^{\prime}\right)$ does not cover entirely $I(l, s, j)$ we enlarge $\omega_{l, s, j}^{\prime}$ gluing it with its adjacent intervals in (3.5), getting a new decomposition of $\omega \backslash f^{-n}(\mathcal{C})$ into intervals $\omega_{l, s, j}$ such that

$$
\begin{aligned}
I(l, s, j) \subset f^{n}\left(\omega_{l, s, j}\right) \subset I(l, s, j)^{+} & \\
& \text {for }|l| \geq k_{0},|s| \geq 1,1 \leq j \leq(|l|+|s|)^{3} .
\end{aligned}
$$

We put $\omega_{l, s, j} \in \mathcal{P}_{n}$ for all $(l, s, j)$ such that $\omega_{l, s, j} \neq \emptyset$, with $|l| \geq k_{0}$. This results in a refinement of $\mathcal{P}_{n-1}$ at $\omega$.

We set $Q_{n}\left(\omega_{l, s, j}\right)=Q_{n-1}(\omega) \cup\{(l, s, j)\}$ for every non-empty interval $\omega_{l, s, j}$. The interval $I(l, s, j)^{+}$is the host interval of $\omega_{l, s, j}$. We define $R_{n}\left(\omega_{l, s, j}\right) \cup\{n\}$ and we say that $n$ is an

(i) escape time for $\omega_{l, s, j}$ if $|l| \geq k_{0}$ and $s \leq s(\tau)$,

(ii) essential return time for $\omega_{l, s, j}$ if $|l| \geq k_{0}$ and $s>s(\tau)$.

REMARK 3.3. We note that if $n$ is a free time or an escape time for $z$, then $x=f^{n}(z)$ either is in the region $\mathbb{S}^{1} \backslash[-\varepsilon, \varepsilon]$ and thus $\left|f^{\prime}(x)\right| \gg 1$, or 
satisfies the inequality (3.4). Hence at free times and escape times we always have expansion of derivatives bounded from below by some uniform constant $\sigma_{0}>1$. We stress that we may and will assume that $\sigma_{0}>\max \{e, \sqrt{\tilde{\sigma}}\}$ in what follows.

To complete the induction step, all we need is to check that (3.1) holds for $\mathcal{P}_{n}$. Since for any interval $J \subset \mathbb{S}^{1}$,

$$
\left.\begin{array}{l}
\left.f^{n}\right|_{J} \text { is a diffeomorphism } \\
\mathcal{C} \cap f^{n}(J)=\emptyset
\end{array}\right\}\left.\Rightarrow f^{n+1}\right|_{J} \text { is a diffeomorphism, }
$$

all we are left to prove is that $\mathcal{C} \cap f^{n}(\omega)=\emptyset$ for all $\omega \in \mathcal{P}_{n}$.

Let $\omega \in \mathcal{P}_{n}$. If $n$ is a free time for $\omega$ then we are done. If $n$ is either a return time for $\omega$, essential or inessential, or an escape time, then by construction we have $f^{n}(\omega) \subset I(l, s, j)^{+}$for some $|l| \geq k_{0},|s| \geq 1, j=1, \ldots,(|l|+|s|)^{3}$ (or for $(l, s, j)=(0,0,0))$ and thus $\mathcal{C} \cap f^{n}(\omega)=\emptyset$. For the binding case we use the following estimate.

Proposition 3.4. Let $n \geq 1$ and $\omega \in \mathcal{P}_{n}$ be such that $n$ is a binding time for $\omega$. Then either $\left|f^{n}(x)\right|>x_{k_{0}}$ or $\operatorname{dist}\left(f^{n}(x), \mathcal{C}\right) \geq \rho_{0} e^{-\rho(n-r)}$ for all $x \in \omega$, where $r=r_{\gamma(n-1)}$ is the last return time for $\omega$ with $n<r+p\left(f^{r}(\omega)\right)$ and $\rho_{0}=1-e^{-\rho}$. Moreover, there is no element of $\mathcal{C}$ between $f^{n}(x)$ and $f^{n-r}\left(x_{l_{r}}\right)$, where $x_{l_{r}}$ is the critical point associated to the return at time $r$.

This result is enough to conclude that $\mathcal{C} \cap f^{n}(\omega)=\emptyset$, completing the induction step.

Proof. We know, from item (1b) of Theorem 1.1, that for $\mu \in S$, every $h \geq 1$ and all $|l| \geq k_{0}$, either

$$
\left|f^{h}\left(f\left(x_{l}\right)\right)\right|>\varepsilon \quad \text { or } \quad\left|f^{h}\left(f\left(x_{l}\right)\right)-x_{m(h)}\right| \geq e^{-\rho h},
$$

where $x_{m(h)}$ is the critical point closest to $f^{h}\left(f\left(x_{l}\right)\right)$ as before. In the former case, if $n$ is a binding time for $\omega$, by the the definition of binding period, for all $x \in \omega$ we get

$$
\begin{aligned}
\left|f^{n}(x)\right| & \geq\left|f^{n-r_{\gamma(n-1)}}\left(x_{l_{\gamma(n-1)}}\right)\right|-\left|f^{n}(x)-f^{n-r_{\gamma(n-1)}}\left(x_{l_{\gamma(n-1)}}\right)\right| \\
& \geq \varepsilon-\varepsilon^{1+\tau} e^{-\tau\left(n-r_{\gamma(n-1)}\right)} \geq \frac{\varepsilon}{x_{k_{0}}}\left(1-\varepsilon^{\tau}\right) x_{k_{0}} \\
& =2 \frac{1-\varepsilon^{\tau}}{1+e^{-\pi / \beta}} x_{k_{0}}>x_{k_{0}},
\end{aligned}
$$

according to condition (1.3) on the choice of $k_{0}$ as a function of $\tau$.

In the latter case in (3.6), by definition of binding (3.3) and because we assume that $\rho<\tau$, setting $m=m\left(n-1-r_{\gamma(n-1)}\right)$ for simplicity, we see 
that $\left|f^{n}(x)-x_{m}\right|$ is bounded by

$$
\begin{aligned}
& \mid f^{n-r_{\gamma(n-1)}\left(x_{l_{\gamma(n-1)}}\right)-x_{m}|-| f^{n}(x)-f^{n-r_{\gamma(n-1)}}\left(x_{l_{\gamma(n-1)}}\right) \mid} \\
& \quad \geq e^{-\rho\left(n-1-r_{\gamma(n-1)}\right)}-\left|f^{\left(n-r_{\gamma(n-1)}\right)}\left(x_{l_{\gamma(n-1)}}\right)-x_{m}\right| e^{-\tau\left(n-r_{\gamma(n-1)}\right)} \\
& \quad \geq e^{-\rho\left(n-r_{\gamma(n-1)}\right)}(1-\varepsilon)>0
\end{aligned}
$$

To complete the proof we consider the case when $x_{l_{\gamma(n-1)}}$ is not the closest critical point to $f^{r(n-1)}(x)$. We first argue that no $x^{\prime} \in \mathcal{C}$ is between $f^{n}(x)$ and $f^{n-r_{\gamma(n-1)}}\left(x_{l_{\gamma(n-1)}}\right)$. For otherwise using (3.3) and the definition of $x_{l_{\gamma(n-1)}}$ we would have

$$
\begin{aligned}
\frac{1}{2}\left|x^{\prime}-x_{m}\right| & <\left|f_{\mu}^{n}(x)-f^{n-r_{\gamma(n-1)}}\left(x_{l_{\gamma(n-1)}}\right)\right| \\
& \leq \mid f^{n-r_{\gamma(n-1)}\left(x_{l_{\gamma(n-1)}}\right)-x_{m} \mid e^{-\tau\left(n-r_{\gamma(n-1)}\right)}} \\
& \leq \frac{e^{-\tau\left(n-r_{\gamma(n-1)}\right)}}{2}\left|x^{\prime}-x_{m}\right|,
\end{aligned}
$$

a contradiction because $e^{-\tau\left(n-r_{\gamma(n-1)}\right)}<1$. Hence there exists $x^{\prime} \in \mathcal{C}$ such that $x^{\prime}$ and $x_{m}$ are consecutive critical points in $\mathcal{C}$ and both $f_{\mu}^{n}(x)$ and $f^{n-r_{\gamma(n-1)}}\left(x_{l_{\gamma(n-1)}}\right)$ are between $x^{\prime}$ and $x_{m}$. But then

$$
\begin{aligned}
\left|x^{\prime}-f^{n}(x)\right| & \geq\left|x^{\prime}-f^{n-r_{\gamma(n-1)}}\left(x_{l_{\gamma(n-1)}}\right)\right|-\left|f^{n}(x)-f^{n-r_{\gamma(n-1)}}\left(x_{l_{\gamma(n-1)}}\right)\right| \\
& \geq \frac{1}{2}\left|x^{\prime}-x_{m}\right|-\left|f^{n-r_{\gamma(n-1)}}\left(x_{l_{\gamma(n-1)}}\right)-x_{m}\right| e^{-\tau\left(n-r_{\gamma(n-1)}\right)} \\
& \geq \frac{1}{2}\left|x^{\prime}-x_{m}\right|-\frac{1}{2}\left|x^{\prime}-x_{m}\right| e^{-\tau\left(n-r_{\gamma(n-1)}\right)} \\
& \geq \frac{1}{2}\left|x^{\prime}-x_{m}\right|\left(1-e^{-\tau\left(n-r_{\gamma(n-1)}\right)}\right) .
\end{aligned}
$$

We observe that since $x^{\prime}$ and $x_{m}$ are consecutive critical points, $x^{\prime}$ is either $x_{m+1}$ or $x_{m-1}$, thus

$$
\left|x^{\prime}-x_{m}\right| \geq 2\left|f^{n-r_{\gamma(n-1)}}\left(x_{l_{\gamma(n-1)}}\right)-x_{m}\right| \geq 2 e^{-\rho\left(n-r_{\gamma(n-1)}\right)} .
$$

Combining the last two inequalities and taking into account that $\rho<\tau$ gives

$$
\begin{aligned}
\left|x^{\prime}-f^{n}(x)\right| & \geq e^{-\rho\left(n-r_{\gamma(n-1)}\right)}\left(1-e^{-\tau\left(n-r_{\gamma(n-1)}\right)}\right) \\
& \geq e^{-\rho\left(n-r_{\gamma(n-1)}\right)}\left(1-e^{-\rho\left(n-r_{\gamma(n-1)}\right)}\right) .
\end{aligned}
$$

Choosing $\rho$ and $\varepsilon$ close to 0 such that $e^{-\rho}>\varepsilon(\rho<\log 2$ and $\varepsilon<1 / 2$ is enough) we get $1-\varepsilon>1-e^{-\rho}$ and we may then replace $1-\varepsilon$ by $\rho_{0}=1-e^{-\rho}$ in (3.7), finishing the proof since $1-e^{-\rho\left(n-r_{\gamma(n-1)}\right)} \geq 1-e^{-\rho}=\rho_{0}$. 
4. Auxiliary lemmas. Here we collect some intermediate results needed for the proofs of the main estimates. In all that follows we write $C$ for a constant depending only on the initial map $\hat{f}$ or $f_{0}$.

Lemma 4.1 ([PRV, Lemma 3.1]). Given $\alpha_{1}, \alpha_{2}, \beta_{1}, \beta_{2}$ with $\alpha_{1} / \alpha_{2} \neq$ $\beta_{1} / \beta_{2}$, there exists $\delta>0$ such that, for every $x$, at least one of the following assertions hold: $\left|\alpha_{1} \sin x+\beta_{1} \cos x\right| \geq \delta$ or $\left|\alpha_{2} \sin x+\beta_{2} \cos x\right| \geq \delta$.

Using this we obtain the following property of bounded distortion for the second derivative near critical points. Observe that since there are inflection points between consecutive critical points, the lower bound cannot hold in general, so we restrict this bound to a small scaled neighborhood of the critical set, reducing the value of $\tau>0$ if needed.

Lemma 4.2. There exists $\tau>0$ small enough and a constant $C>0$ depending only on $\hat{f}$ such that for every $k \geq k_{0}$ and $t \in\left[y_{k+1}, y_{k}\right]$ we have $\left|f^{\prime \prime}(t)\right| /\left|f^{\prime \prime}\left(x_{k}\right)\right| \leq C$.

Moreover, we can find a constant $C$, depending only on $\hat{f}$ and $\tau$, such that for $\left|t-x_{k}\right|<\tau\left|x_{k}\right|$ we have $\left|f^{\prime \prime}(t)\right| /\left|f^{\prime \prime}\left(x_{k}\right)\right| \geq C^{-1}$.

Note that the condition on the lower bound above is satisfied by all points in a return situation, either essential or inessential, during the construction of the sequence of partitions $\mathcal{P}_{n}$, as detailed in the previous Section 3.

Proof. Since $f$ is symmetric we can assume without loss that $z>0$ in what follows. We compute

$$
\begin{aligned}
& f^{\prime}(z)=-a z^{\alpha-1}[\alpha \sin (\beta \log z)+\beta \cos (\beta \log z)], \\
& f^{\prime \prime}(x)=-a z^{\alpha-2}[A \sin (\beta \log z)+B \cos (\beta \log z)],
\end{aligned}
$$

where $A=\alpha(\alpha-1)-\beta^{2}$ and $B=\beta(2 \alpha-1)$. Note that for $z=x_{l}$ we have

$$
0=f^{\prime}\left(x_{l}\right)=\alpha \sin \left(\beta \log x_{l}\right)+\beta \cos \left(\beta \log x_{l}\right)
$$

and

$$
\frac{\alpha}{A} \neq \frac{\beta}{B} \Leftrightarrow \alpha^{2}+\beta^{2} \neq 0, \quad \text { which is true, since } \alpha, \beta>0 .
$$

Applying Lemma 4.1 we get, because $f^{\prime}\left(x_{k}\right)=0$,

$$
\frac{\left|f^{\prime \prime}(t)\right|}{\left|f^{\prime \prime}\left(x_{k}\right)\right|} \leq\left|\frac{x_{k+1}}{x_{k}}\right|^{\alpha-2} \frac{|A|+|B|}{\delta} \leq e^{-(\pi / \beta)(\alpha-2)} \frac{|A|+|B|}{\delta},
$$

where the last inequality is a direct consequence of (1.2).

Now for the lower bound we compute the zeroes of $f^{\prime \prime}$ and obtain $z_{k}=\hat{z} \exp \left(-k \frac{\pi}{\beta}\right) \quad$ for $k \geq k_{0}, \quad$ where $\hat{z}=\exp \left(\frac{1}{\beta} \tan ^{-1} \frac{\beta(2 \alpha-1)}{\beta^{2}-\alpha^{2}+\alpha}\right)$. By the expressions for the zeroes of $f^{\prime}$ and the zeroes of $f^{\prime \prime}$ we see that their distance is scaled by a common factor. Thus if we consider a scaled 
neighborhood of each $x_{l}$ specified by a small enough $\tau>0$, that is, considering only $t$ with $\left|t-x_{l}\right|<\tau\left|x_{l}\right|$, then we ensure that $t$ is far away from the inflection point.

Moreover, the value of the quotient in the statement of the lemma is invariant under the scaling: let $l>k \geq k_{0}$ and $\left|t-x_{k}\right|<\tau\left|x_{k}\right|$. Then on the one hand,

$$
e^{-(l-k) \pi / \beta}\left|t-x_{k}\right|=\left|s-x_{l}\right|<\tau\left|x_{l}\right| \quad \text { where } \quad s=t e^{-(l-k) \pi / \beta} .
$$

On the other hand,

$$
\begin{aligned}
& \frac{\left|f^{\prime \prime}(s)\right|}{\left|f^{\prime \prime}\left(x_{l}\right)\right|}=\frac{\left|a s^{\alpha-2}[A \sin (\beta \log s)+B \cos (\beta \log s)]\right|}{\left|a x_{l}^{\alpha-2}\left[A \sin \left(\beta \log x_{l}\right)+B \cos \left(\beta \log x_{l}\right)\right]\right|} \\
& =\frac{\left|a\left(t e^{-(l-k) \pi / \beta}\right)^{\alpha-2}[A \sin (\beta \log t+(k-l) \pi)+B \cos (\beta \log t+(k-l) \pi)]\right|}{\left|a\left(x_{k} e^{-(l-k) \pi / \beta}\right)^{\alpha-2}\left[A \sin \left(\beta \log x_{k}+(k-l) \pi\right)+B \cos \left(\beta \log x_{k}+(k-l) \pi\right)\right]\right|}
\end{aligned}
$$

is the same value of $\left|f^{\prime \prime}(t)\right| /\left|f^{\prime \prime}\left(x_{k}\right)\right|$. Therefore the lower bound is given by

$$
\inf \left\{\frac{\left|f^{\prime \prime}(t)\right|}{\left|f^{\prime \prime}\left(x_{k}\right)\right|}:\left|t-x_{k}\right|<\tau\left|x_{k}\right|\right\}
$$

for any $k \geq k_{0}$. Taking $C>0$ large enough we conclude the proof.

The next result guarantees that orbits of points in $\left[-C \varepsilon^{\alpha},-\tilde{y}\right] \cup\left[\tilde{y}, C \varepsilon^{\alpha}\right]$ remain expanding during a number $m_{0}$ of iterates that can be fixed arbitrarily large by reducing $\varepsilon$ and $\mu$. Recall that $\left|\tilde{f}^{\prime}\right|>\sigma_{0} \gg 1$ and that $f$ is $C^{1}$-close to $\tilde{f}$ outside $[-\tilde{y}, \tilde{y}]$ if $\mu$ is small.

Lemma 4.3 ([PRV, Lemma 6.1]). There exist $c, C>0$ and $m_{0} \geq c \log (1 / \varepsilon)$ such that if $\tilde{y}<|x| \leq C \varepsilon^{\alpha}$, then $f^{i}(x) \notin[-\tilde{y}, \tilde{y}]$ and $\left|f^{\prime}\left(f^{i}(x)\right)\right| \geq \sigma_{0}$ for all $1 \leq i \leq m_{0}$ and all $\mu \in[-\varepsilon, \varepsilon]$.

Next we establish some results of bounded distortion and uniformly bounded expansion during binding periods.

Lemma 4.4 (Bounded distortion on binding periods). There exists $A=$ $A(C, \tau)>1$ such that for all $x \in I(l, s)$ we have

$$
\frac{1}{A} \leq\left|\frac{\left(f^{j}\right)^{\prime}(\xi)}{\left(f^{j}\right)^{\prime}\left(f\left(x_{l}\right)\right)}\right| \leq A
$$

for every $1 \leq j \leq p(l, s)$ and every $\xi \in\left[f\left(x_{l}\right), f(x)\right]$.

Proof. We let $\eta=f\left(x_{l}\right)$ and consider $0 \leq i<j$. There are two cases to treat, corresponding to the two possibilities in (3.3). If $\left|f^{i}(\eta)\right| \leq \varepsilon$ then, by Lemma 1.4,

$$
\left|\frac{f^{\prime}\left(f^{i}(\xi)\right)-f^{\prime}\left(f^{i}(\eta)\right)}{f^{\prime}\left(f^{i}(\eta)\right)}\right| \leq C \frac{\left|f^{i}(\xi)-f^{i}(\eta)\right|}{\left|f^{i}(\eta)-x_{m(i)}\right|} \leq C e^{-\tau i} .
$$


If $\left|f^{i}(\eta)\right|>\varepsilon$, then $\left|f^{i}(\xi)-f^{i}(\eta)\right| \leq \varepsilon^{1+\tau} e^{-\tau i} \ll \varepsilon$ and so the interval bounded by $f^{i}(\xi)$ and $f^{i}(\eta)$ is contained in the region $\mathbb{S}^{1} \backslash[-\tilde{y}, \tilde{y}]$, where $f=\tilde{f}$. Thus,

$$
\left|\frac{f^{\prime}\left(f^{i}(\xi)\right)-f^{\prime}\left(f^{i}(\eta)\right)}{f^{\prime}\left(f^{i}(\eta)\right)}\right| \leq C\left|f^{i}(\xi)-f^{i}(\eta)\right| \leq C \varepsilon^{1+\tau} e^{-\tau i} \leq C e^{-\tau i} .
$$

Putting together all the above we get

$$
\sum_{i=0}^{j-1}\left|\frac{f^{\prime}\left(f^{i}(\xi)\right)-f^{\prime}\left(f^{i}(\eta)\right)}{f^{\prime}\left(f^{i}(\eta)\right)}\right| \leq C \sum_{i=0}^{j} e^{-\tau i} \leq C .
$$

Thus

$$
\log \left|\frac{\left(f^{j}\right)^{\prime}(\xi)}{\left(f^{j}\right)^{\prime}(\eta)}\right| \leq \sum_{i=0}^{j-1} \log \left(1+\left|\frac{f^{\prime}\left(f^{i}(\xi)\right)}{f^{\prime}\left(f^{i}(\eta)\right)}-1\right|\right) \leq \sum_{i=0}^{j-1}\left|\frac{f^{\prime}\left(f^{i}(\xi)\right)}{f^{\prime}\left(f^{i}(\eta)\right)}-1\right| \leq C,
$$

and the statement of the lemma follows.

Now we prove an exponential bound on the derivative along the orbit of each critical value $z_{k}$ with $|k| \geq k_{0}$. This is needed to get a lower bound for the binding time $p$ in terms of the position of the return interval given by $(l, s)$ in the following Lemma 4.6. This demands a proof since the derivative of $f$ is unbounded due to the presence of infinitely many critical points, unlike the quadratic family where we have this property for free.

In our setting we will obtain this by imposing an extra condition, besides item (1b) from Theorem 1.1, in the construction of the set $S$ of parameters $\mu$ which we shall consider in the proof of Theorems B and C. This condition is expressed by the inequality

$$
\sum_{j=0}^{n-1}-\log \operatorname{dist}\left(f_{\mu}^{j}\left(z_{k}\right), \mathcal{C}\right) \leq \hat{M} n,
$$

for all $n \geq 1$ which are not bound times for the orbit of the critical value $z_{k}$, for every $|k| \geq k_{0}$ and for some fixed large constant $\hat{M}>0$. Since for all $x \in I$ we have $|x| \geq \operatorname{dist}(x, \mathcal{C})$ this implies

$$
\sum_{j=0}^{n-1}-\log \left|f_{\mu}^{j}\left(z_{k}\right)\right| \leq \hat{M} n
$$

and we are able to deduce the following.

Lemma 4.5. Assume that (4.2) holds for some $\mu \in S$. Then there exists a constant $M>0$ such that $\left|\left(f^{n}\right)^{\prime}\left(z_{k}\right)\right| \leq M^{n}$ for all $n \geq 1$ and $|k| \geq k_{0}$.

Proof. Note that just by taking the derivative of $f$ we see that there exists a constant $C>0$ such that $\left|f^{\prime}(x)\right| \leq C|x|^{\alpha-1}$. On the one hand, for $n \geq 1$ such that $n$ is not a bound time for $z_{k}$ and $|k| \geq k_{0}$ we use (4.2) to 
get

$$
\begin{aligned}
\left|\left(f^{n}\right)^{\prime}\left(z_{k}\right)\right| & \leq \prod_{j=0}^{n-1} C\left|f_{\mu}^{j}\left(z_{k}\right)\right|^{\alpha-1} \\
& \leq \exp \left(\sum_{j=0}^{n-1}\left(\log C+(\alpha-1) \log \left|f_{\mu}^{j}\left(z_{k}\right)\right|\right)\right) \\
& \leq \exp (n \log C+(1-\alpha) \hat{M} n)=\tilde{M}^{n}
\end{aligned}
$$

where $\tilde{M}=\exp (\log C+(1-\alpha) \hat{M})$. We can assume without loss that $\tilde{M}>A$ where $A>1$ is given by Lemma 4.4 .

On the other hand, if $n$ is a bound time for $z_{k}$, let $t_{1}<n$ be the return time for $z_{k}$ which originated the binding and $z_{l_{1}}$ be the corresponding bound critical value. Then by Lemma 4.4 ,

$$
\left|\left(f^{n}\right)^{\prime}\left(z_{k}\right)\right| \leq A\left|\left(f^{n-t_{1}}\right)^{\prime}\left(z_{l_{1}}\right)\right| \cdot\left|\left(f^{t_{1}}\right)^{\prime}\left(z_{k}\right)\right| .
$$

If $t_{1}$ is not a bound time for $z_{l_{1}}$, then by the bound just proved for all critical values on free times and return situations we bound the last expression by $A \tilde{M}^{n-t_{1}} \tilde{M}^{t_{1}}=A \tilde{M}^{n}$. Otherwise there are $t_{2}<n-t_{1}$, the return time for $z_{l_{1}}$ which originated the binding, and $z_{l_{2}}$, the corresponding bound critical value, and as above we get

$$
\left|\left(f^{n}\right)^{\prime}\left(z_{k}\right)\right| \leq A^{2}\left|\left(f^{n-t_{1}-t_{2}}\right)^{\prime}\left(z_{l_{2}}\right)\right| \cdot\left|\left(f^{t_{2}}\right)^{\prime}\left(z_{l_{1}}\right)\right| \cdot\left|\left(f^{t_{1}}\right)^{\prime}\left(z_{k}\right)\right| .
$$

If $n-t_{1}-t_{2}$ is not a bound time for $z_{l_{2}}$ we bound this expression by $A^{2} \tilde{M}^{n-t_{1}-t_{2}} \tilde{M}^{t_{2}} \tilde{M}^{t_{1}} \leq A^{2} \tilde{M}^{n}$. Otherwise we repeat the argument. Knowing that the orbit of every critical value has an initial number $j_{0} \gg 1$ of free iterates [PRV, beginning of Section 4, p. 450], we see that this argument must end in a free time and we arrive at $\left|\left(f^{n}\right)^{\prime}\left(z_{k}\right)\right| \leq A^{l} \tilde{M}^{n}$ where $l<n$ is the number of nested binding periods obtained. Since $\tilde{M}>A$ we conclude the proof of the lemma by setting $M=\tilde{M}^{2}$.

Now we obtain the estimates for the binding time assuming that (4.2) holds for $\mu \in S$. In Section 8 we explain how to obtain (4.2) for a positive measure subset of parameters in $S$.

LEMMA 4.6 (Expansion during binding periods). There are constants $A_{0}=A_{0}(\varepsilon, \rho, \tau)>1, \iota=\iota(M)>0$ and $\theta=\theta(M, \varepsilon, \rho, \tau) \in \mathbb{N}$ such that for $n \geq 1$ and $\omega \in \mathcal{P}_{n}$ with $R_{n}(\omega) \neq \emptyset$, if $r$ is the last return time for $\omega$ and $f^{r}(\omega) \subset I(l, s, j)$, then setting $p=p(l, s)>0$ and $\zeta=2(\rho+\tau) / \log \sigma$ we have, for $\tau$ small enough:

(a) $\iota(M)(|l|+|s|) \leq p \leq \frac{2 \pi}{\beta \log \sigma}(|l|+|s|)$; 
(b) $\left|\left(f^{p+1}\right)^{\prime}\left(f^{r}(x)\right)\right| \geq \frac{1}{C} \varepsilon^{1+\tau} \exp \left((1-\zeta) \frac{\pi}{\beta}(|l|+|s|)\right)$

and if $|l|+|s| \geq \theta$, then

$$
\left|\left(f^{p+1}\right)^{\prime}\left(f^{r}(x)\right)\right| \geq \frac{1}{C} \exp \left(\frac{\pi}{\beta}(|l|+|s|)\right),
$$

for every $x \in \omega$

(c) $\left|\left(f^{p+1}\right)^{\prime}\left(f^{r}(x)\right)\right| \geq A_{0} \sigma^{(p+1) / 3}>2$ for every $x \in \omega$.

Proof. To prove item (a), we use the definition of the partition and the construction of the refinement. As $p>0,(l, s, j) \neq\left( \pm k_{0}, 1,1\right)$ and so $\left|f^{r}(x)-x_{l}\right| \geq a_{2} e^{-(\pi / \beta)(|l|+|s|)}$, by Remark 2.1 , where $x$ is any given point in $\omega$. Using the second order Taylor approximation and Lemma 4.2 we get

$$
\begin{aligned}
\left|f^{r+1}(x)-f\left(x_{l}\right)\right| & \geq \frac{1}{C}\left|f^{\prime \prime}\left(x_{l}\right)\right|\left(a_{2} e^{-(\pi / \beta)(|l|+|s|)}\right)^{2} \\
& \geq \frac{1}{C} e^{-(\pi / \beta)|l|(\alpha-2)} e^{-2(\pi / \beta)(|l|+|s|)},
\end{aligned}
$$

where $\left|f^{\prime \prime}\left(x_{l}\right)\right| \geq C^{-1}\left|x_{l}\right|^{\alpha-2}=C^{-1} \hat{x}^{\alpha-2} e^{-(\pi / \beta)|l|(\alpha-2)}$ by Lemma 1.3(2). Then for each $0 \leq j \leq p$, there is some $\xi$ between $f\left(x_{l}\right)$ and $f^{r+1}(x)$ such that

$$
\begin{aligned}
\left|f^{j+r+1}(x)-f^{j+1}\left(x_{l}\right)\right| & =\left|\left(f^{j}\right)^{\prime}(\xi)\right| \cdot\left|f^{r+1}(x)-f\left(x_{l}\right)\right| \\
& \geq C^{-1} e^{-(\pi / \beta)|l|(\alpha-2)} e^{-2(\pi / \beta)(|l|+|s|)}\left|\left(f^{j}\right)^{\prime}(\xi)\right| .
\end{aligned}
$$

Now since $\alpha-2<0$ and we can take $|l| \geq k_{0}$ very large, as a consequence of Lemma 4.4 and of the exponential growth of the derivative at the critical orbits, we get the bound

$$
2 e^{-2(\pi / \beta)(|l|+|s|)} \sigma^{j} \leq\left|f^{j+r+1}(x)-f^{j+1}\left(x_{l}\right)\right| \leq 2 .
$$

Hence $e^{-2(\pi / \beta)(|l|+|s|)} \sigma^{j} \leq 1$ for all $1 \leq j \leq p$. In particular,

$$
-2(\pi / \beta)(|l|+|s|)+p \log (\sigma) \leq 0, \quad \text { implying } \quad p \leq \frac{2(\pi / \beta)(|l|+|s|)}{\log \sigma},
$$

thus proving the upper bound in (a).

For the lower bound in (a), we note that by the definition of binding period, we have

$$
\begin{aligned}
\mid f^{p+r+1}(x)- & f^{p+1}\left(x_{l}\right) \mid \\
& \geq \begin{cases}\left|f^{p+1}\left(x_{l}\right)-x_{m(p)}\right| e^{-\tau(p+1)} & \text { if }\left|f^{p+1}\left(x_{l}\right)\right| \leq \varepsilon \\
\varepsilon^{1+\tau} e^{-\tau(p+1)} & \text { if }\left|f^{p+1}\left(x_{l}\right)\right|>\varepsilon\end{cases}
\end{aligned}
$$


So in either case using Lemmas 4.4 and 4.5 we get

$$
\begin{aligned}
\left|f^{p+r+1}(x)-f^{p+1}\left(x_{l}\right)\right| & =\left|\left(f^{p}\right)^{\prime}(\xi)\right| \cdot\left|f^{r+1}(x)-f\left(x_{l}\right)\right| \\
& \leq A\left|\left(f^{p}\right)^{\prime}\left(z_{l}\right)\right| \cdot\left|f^{r+1}(x)-f\left(x_{l}\right)\right| \\
& \leq A M^{p} C\left|x_{l}\right|^{\alpha-2}\left|f^{r}(x)-x_{l}\right|^{2} \\
& \leq A M^{p} C e^{-(\pi / \beta)(\alpha|l|+2|s|)} \leq A M^{p} e^{-\alpha(\pi / \beta)(|l|+|s|)},
\end{aligned}
$$

where $\xi$ is some point between $f^{r+1}(x)$ and $f\left(x_{l}\right)$ and in the third line above we have used Lemma 1.3.

On the one hand, if $\left|f^{p+1}\left(x_{l}\right)\right|>\varepsilon$ then we arrive at $\varepsilon^{1+\tau} e^{-\tau(p+1)} \leq$ $A M^{p} e^{-\alpha(\pi / \beta)(|l|+|s|)}$. On the other hand, if $\left|f^{p+1}\left(x_{l}\right)\right| \leq \varepsilon$ then by condition (1b) from Theorem 1.1 we arrive at $e^{-\rho p} e^{-\tau(p+1)} \leq A M^{p} e^{-\alpha(\pi / \beta)(|l|+|s|)}$. In both cases if we take $M$ large enough, then we get a bound of the form $p \geq \iota(M)(|l|+|s|)$ concluding the proof of (a).

Now we prove (b). Since $p+1$ is not a binding time we must have (4.4) as before. Using Theorem 1.1(1b), setting

$$
\Delta_{p+1}=\min \left\{\varepsilon^{1+\tau} e^{-\tau(p+1)}, e^{-(\rho+\tau)(p+1)}\right\}
$$

we get for some $\xi \in\left[f\left(x_{l}\right), f^{r+1}(x)\right]$, by Lemma 4.4, and using second order Taylor expansion of $f$ near $x_{l}$ together with the upper bound from Lemma 4.2,

$$
\begin{aligned}
\Delta_{p+1} & \leq\left|f^{p+1}\left(x_{l}\right)-f^{p+r+1}(x)\right|=\left|\left(f^{p}\right)^{\prime}(\xi)\right| \cdot\left|f\left(x_{l}\right)-f^{r+1}(x)\right| \\
& \leq C\left|\left(f^{p}\right)^{\prime}\left(f^{r+1}(x)\right)\right| \cdot\left|f^{\prime \prime}\left(x_{l}\right)\right| \cdot\left|f^{r}(x)-x_{l}\right|^{2} .
\end{aligned}
$$

Note that the second order Taylor expansion near $x_{l}$ gives

$$
\begin{aligned}
\left|f\left(x_{l}\right)-f^{r+1}(x)\right| & =\left|f^{\prime}\left(x_{l}\right)\left(x_{l}-f^{r}(x)\right)+\frac{f^{\prime \prime}(\xi)}{2}\left(x_{l}-f^{r}(x)\right)^{2}\right| \\
& =\left|\frac{f^{\prime \prime}(\xi)}{2}\left(x_{l}-f^{r}(x)\right)^{2}\right|
\end{aligned}
$$

for some $\xi$ between $x_{l}$ and $f^{r}(x)$. Together with Lemma 4.2 we obtain the bound in (4.5).

On the other hand, using Lemma 1.3 and again Lemma 4.4 we get

$$
\begin{aligned}
\left|\left(f^{p+1}\right)^{\prime}\left(f^{r}(x)\right)\right| & =\left|f^{\prime}\left(f^{r}(x)\right)\right| \cdot\left|\left(f^{p}\right)^{\prime}\left(f^{r+1}(x)\right)\right| \\
& \geq C^{-1}\left|x_{l}\right|^{\alpha-2}\left|f^{r}(x)-x_{l}\right| \cdot\left|\left(f^{p}\right)^{\prime}\left(f^{r+1}(x)\right)\right| .
\end{aligned}
$$

Hence by the previous expression together with (4.5) we deduce

$$
\left|\left(f^{p+1}\right)^{\prime}\left(f^{r}(x)\right)\right| \geq \frac{\varepsilon^{1+\tau} e^{-(\rho+\tau)(p+1)}}{C\left|f^{r}(x)-x_{l}\right|} \geq \frac{1}{C} \varepsilon^{1+\tau} e^{-(\rho+\tau)(p+1)} e^{(\pi / \beta)(|l|+|s|)} .
$$

Now we have two possibilities: either $\varepsilon>e^{-(\rho+\tau)(p+1)}$ or not. In the former case we obtain, by the upper bound in item (a) and because we can assume 
that $2+\tau \leq 3$ and $\rho+\tau \leq 1$,

$$
\left|\left(f^{p+1}\right)^{\prime}\left(f^{r}(x)\right)\right| \geq e^{-(2+\tau)(\rho+\tau)(p+1)} e^{(\pi / \beta)(|l|+|s|)} \geq \frac{1}{C} \exp \left(\frac{\pi}{\beta}(|l|+|s|)\right) .
$$

In the latter case we get

$$
\left|\left(f^{p+1}\right)^{\prime}\left(f^{r}(x)\right)\right| \geq C^{-1} \varepsilon^{1+\tau} \exp \left(\left(1-\frac{\rho+\tau}{\log \sigma}\right) \frac{\pi}{\beta}(|l|+|s|)\right) .
$$

Observe that in this case $\log \varepsilon \leq-(\rho+\tau)(p+1)$, thus by the lower bound in item (a) and since $k_{0}=C \log (1 / \varepsilon)$ we have

$$
(\rho+\tau) \iota(M)(|l|+|s|+1) \leq(\rho+\tau)(p+1) \leq C k_{0} .
$$

So if $|l|+|s| \geq \theta$ with $(\rho+\tau) \iota(M)(\theta+1)>C k_{0}$ then only the first alternative can happen. This concludes the proof of item (b).

In order to prove (c) we use Lemma 4.4 once again and the Mean Value Theorem applied to $f^{\prime}$ near $x_{l}$ together with the lower bound from Lemma 4.2 to get

$$
\begin{aligned}
& \left|\left(f^{p+1}\right)^{\prime}\left(f^{r}(x)\right)\right|^{2}=\left|\left(f^{p}\right)^{\prime}\left(f^{r+1}(x)\right)\right|^{2} \cdot\left|f^{\prime}\left(f^{r}(x)\right)\right|^{2} \\
\geq & \left(C^{-1}\left|\left(f^{p}\right)^{\prime}\left(f\left(x_{l}\right)\right)\right| \cdot\left|\left(f^{p}\right)^{\prime}\left(f^{r+1}(x)\right)\right|\right)\left(C^{-1}\left|f^{\prime \prime}\left(x_{l}\right)\right|^{2}\left|f^{r}(x)-x_{l}\right|^{2}\right) \\
= & C^{-1}\left|\left(f^{p}\right)^{\prime}\left(f\left(x_{l}\right)\right)\right| \cdot\left|f^{\prime \prime}\left(x_{l}\right)\right|\left(\left|\left(f^{p}\right)^{\prime}\left(f^{r+1}(x)\right)\right| \cdot\left|f^{\prime \prime}\left(x_{l}\right)\right| \cdot\left|f^{r}(x)-x_{l}\right|^{2}\right) .
\end{aligned}
$$

Note that we can use the lower bound from Lemma 4.2 because $r$ is a return time, not an escape nor a free time. Comparing the last expression (4.6) with (4.5) we see that (4.6) is bounded below by

$$
\begin{aligned}
C^{-1}\left|\left(f^{p}\right)^{\prime}\left(f\left(x_{l}\right)\right)\right| \cdot\left|f^{\prime \prime}\left(x_{l}\right)\right|\left(\left|\left(f^{p}\right)^{\prime}(\xi)\right| \cdot\left|f\left(x_{l}\right)-f^{r+1}(x)\right|\right) \\
\quad=C^{-1}\left|\left(f^{p}\right)^{\prime}\left(f\left(x_{l}\right)\right)\right| \cdot\left|f^{\prime \prime}\left(x_{l}\right)\right| \cdot\left|f^{p+1}\left(x_{l}\right)-f^{r+p+1}(x)\right| .
\end{aligned}
$$

Finally, from Lemma 1.3(2) we deduce the lower bound

$$
C^{-1} \sigma^{p} C^{-1}\left|x_{l}\right|^{\alpha-2}\left|f^{p+1}\left(x_{l}\right)-f^{r+p+1}(x)\right| .
$$

Now we consider two cases.

On the one hand, if $\left|f^{p+1}\left(x_{l}\right)\right|>\varepsilon$ then, by the definition of $p$ in (3.3), we must have $\left|f^{p+1}\left(x_{l}\right)-f^{r+p+1}(x)\right|>\varepsilon^{1+\tau} e^{-\tau(p+1)}$. The bound (4.7) together with $\alpha-2<0$ and $\left|x_{l}\right| \leq \varepsilon$ implies that

$$
\left|\left(f^{p+1}\right)^{\prime}\left(f^{r}(x)\right)\right|^{2} \geq C^{-2} \sigma^{p} \varepsilon^{\alpha-2}\left|f^{p+1}\left(x_{l}\right)-f^{r+p+1}(x)\right| .
$$

Now we can write

$$
\left|\left(f^{p+1}\right)^{\prime}\left(f^{r}(x)\right)\right|^{2} \geq C^{-1} \sigma^{p} \varepsilon^{\alpha-1+\tau} e^{-\tau(p+1)} \geq A_{0}^{2} \sigma^{2(p+1) / 3},
$$

if we fix $\tau<\min \{1-\alpha, \log \sigma / 3\}$ and take $\varepsilon$ small enough.

On the other hand, if $\left|f^{p+1}\left(x_{l}\right)\right| \leq \varepsilon$ then $\left|f^{p+1}\left(x_{l}\right)-f^{r+p+1}(x)\right|>$ $\left|f^{p+1}\left(x_{l}\right)-x_{m(p)}\right| e^{-\tau(p+1)}$ by $(3.3)$. 
Finally, we note that there is only one possibility according to item (1b) of Theorem 1.1, that is, $\left|f^{p+1}\left(x_{l}\right)-x_{m(p)}\right| \geq e^{-\rho p}$, and thus $\mid f^{p+1}\left(x_{l}\right)-$ $f^{r+p+1}(x) \mid>C^{-1} \varepsilon e^{-(\rho+\tau)(p+1)}$. Hence

$$
\left|\left(f^{p+1}\right)^{\prime}\left(f^{r}(x)\right)\right|^{2} \geq C^{-1} \sigma^{p} \varepsilon^{\alpha-2} e^{-(\rho+\tau)(p+1)} \geq A_{0}^{2} \sigma^{2(p+1) / 3}
$$

as long as we take $\varepsilon, \rho$ and $\tau$ small enough. This concludes the proof.

REMARK 4.7. Note that $\iota(M) \rightarrow 0$ as $M \rightarrow \infty$.

Now we will obtain estimates of the length of $\left|f^{n}(\omega)\right|$.

LEMmA 4.8 (Lower bounds on the length at return times). Let $r$ be an essential or an inessential return time for $\omega \in \mathcal{P}_{n-1}$, with host interval $I(l, s, j)^{+}$. Let $p=p(l, s)$ denote the length of its binding period and set

$$
Q=Q(l, s, \tau, \rho)=\frac{\varepsilon^{1+\tau}}{(|l|+|s|)^{3}} e^{\zeta(\pi / \beta)(|l|+|s|)} .
$$

(1) Assuming that $r^{*} \leq n-1$ is the next return situation for $\omega$ (either essential, inessential or an escape), we have

$$
\left|f^{k}(\omega)\right| \geq Q \sigma_{0}^{q} e^{(1-2 \zeta)(\pi / \beta)(|l|+|s|)}\left|f^{r}(\omega)\right|
$$

for $q=k-(r+p+1)$ and every $k$ such that $r+p+1 \leq k \leq r^{*}$. Moreover,

$$
\left|f^{k}(\omega)\right| \geq \sigma_{0}^{q} A_{0} \sigma^{(p+1) / 3}\left|f^{r}(\omega)\right| \geq A_{0}\left|f^{r}(\omega)\right|>\left|f^{r}(\omega)\right| .
$$

(2) If $r$ is the last return time for $\omega$ up to iterate $n-1$ and also an essential return, and $r^{*}$ is a return time for $\omega$, then setting $q=$ $k-(r+p+1)$ we have

$$
\left|f^{k}(\omega)\right| \geq a_{1} Q \sigma_{0}^{q} e^{-2 \zeta(\pi / \beta)(|l|+|s|)} \quad \text { for all } r+p+1 \leq k \leq r^{*} .
$$

Suppose that $r$ is an escape time for $\omega \in \mathcal{P}_{n-1}$.

(3) If $r^{*} \leq n-1$ is the next return situation for $\omega$, then $\left|f^{k}(\omega)\right| \geq$ $\sigma_{0}^{k-r}\left|f^{r}(\omega)\right|$ for every $k$ such that $r<k \leq r^{*}$.

Remark 4.9. Note that $Q=Q(l, s, \tau, \rho) \rightarrow \infty$ as $|l|+|s| \rightarrow \infty$.

Proof. We start by assuming $r^{*} \leq n-1$. By the mean value theorem we have $\left|f^{r^{*}}(\omega)\right| \geq\left|\left(f^{r^{*}-r}\right)^{\prime}\left(f^{r}(\xi)\right)\right| \cdot\left|f^{r}(\omega)\right|$ for some $\xi \in \omega$. Using Remark 3.3 and Lemma 4.6 we get, by setting $q=r^{*}-(r+p+1)$,

$$
\begin{aligned}
\left|f^{r^{*}}(\omega)\right| & \geq\left|\left(f^{q}\right)^{\prime}\left(f^{r+p+1}(\xi)\right)\right| \cdot\left|\left(f^{p+1}\right)^{\prime}\left(f^{r}(\xi)\right)\right| \cdot\left|f^{r}(\omega)\right| \\
& \geq \sigma_{0}^{q} \frac{1}{C} \varepsilon^{1+\tau} e^{(1-\zeta)(\pi / \beta)(|l|+|s|)}\left|f^{r}(\omega)\right| \\
& \geq \sigma_{0}^{q} \frac{\varepsilon^{1+\tau}}{C} e^{\zeta(\pi / \beta)(|l|+|s|)} e^{(1-2 \zeta)(\pi / \beta)(|l|+|s|)}\left|f^{r}(\omega)\right| .
\end{aligned}
$$

Taking into account the definition of $Q$ we obtain the first part of item (1). 
If $r$ is an essential return time for $\omega$, then $I(l, s, j) \subset f^{r}(\omega)$ and $\left|f^{r}(\omega)\right| \geq$ $a_{1} e^{-(\pi / \beta)(|l|+|s|)} /(|l|+|s|)^{3}$, hence

$$
\left|f^{r^{*}}(\omega)\right| \geq \sigma_{0}^{q} \frac{\varepsilon^{1+\tau} e^{\zeta(\pi / \beta)(|l|+|s|)}}{C(|l|+|s|)^{3}} a_{1} e^{-2 \zeta(\pi / \beta)(|l|+|s|)},
$$

and by the definition of $Q$ this proves (2).

For the second part of item (1) just use the inequality from Lemma 4.6(c) in the first line of (4.8).

To get item (3) observe that between the iterate $r$ and $r^{*}-1$ there are only free iterates for $\omega$, thus the estimate follows by the uniform expanding rate at free times.

Altogether this concludes the proof of the lemma for the case $k=r^{*}$. For the other cases $r+p<k \leq r^{*}$ observe that only the number of free iterates after the last bound iterate until $k$ is affected, and this number equals $q=k-(r+p+1)$.

Lemma 4.10 (Bounded distortion). There is a constant $D_{0}=D_{0}(\rho, \tau, \sigma)$ $>0$ such that for $\omega \in \mathcal{P}_{n-1}, n \in \mathbb{N}$, and for every $x, y \in \omega$ we have

$$
\left|\left(f^{n}\right)^{\prime}(x)\right| /\left|\left(f^{n}\right)^{\prime}(y)\right| \leq D_{0} .
$$

Proof. Let

$$
R_{n-1}(\omega)=\left\{r_{1}, \ldots, r_{\gamma}\right\} \quad \text { and } \quad Q_{n-1}(\omega)=\left\{\left(l_{1}, s_{1}, j_{1}\right), \ldots,\left(l_{\gamma}, s_{\gamma}, j_{\gamma}\right)\right\}
$$

be the sets of return situations (essential returns, inessential returns and escapes) and indices of host intervals of $\omega$, respectively, as defined during the construction of the partition. Let $\omega_{i}=f^{r_{i}}(\omega), p_{i}=p\left(l_{i}, s_{i}\right)$ for $i=1, \ldots, \gamma$ and, for $y, z \in \omega$, let $y_{k}=f^{k}(y)$ and $z_{k}=f^{k}(z)$ for $k=0, \ldots, n-1$. Observe that $\omega_{i} \subset I\left(l_{i}, s_{i}, j_{i}\right)^{+}$for all $i$ and

$$
\left|\frac{\left(f^{n}\right)^{\prime}(z)}{\left(f^{n}\right)^{\prime}(y)}\right|=\prod_{k=0}^{n-1}\left|\frac{f^{\prime}\left(z_{k}\right)}{f^{\prime}\left(y_{k}\right)}\right| \leq \prod_{k=0}^{n-1}\left(1+\left|\frac{f^{\prime}\left(z_{k}\right)-f^{\prime}\left(y_{k}\right)}{f^{\prime}\left(y_{k}\right)}\right|\right) .
$$

On free iterates, if $y_{k} \in[-\varepsilon, \varepsilon]$, then by Lemma 1.4,

$$
\left|\frac{f^{\prime}\left(z_{k}\right)-f^{\prime}\left(y_{k}\right)}{f^{\prime}\left(y_{k}\right)}\right| \leq K_{1}\left|\frac{z_{k}-y_{k}}{y_{k}-\tilde{x}_{k}}\right| \leq K_{1} \frac{\left|f^{k}(\omega)\right|}{\Delta_{k}(\omega)},
$$

where we define $\Delta_{k}(\omega)=\operatorname{dist}\left(f^{k}(\omega), \mathcal{C}\right)=\inf _{x \in \omega} \operatorname{dist}\left(f^{k}(x), \mathcal{C}\right)$ and $\tilde{x}_{k}$ is the critical point closest to $y_{k}$. We observe that in this case the interval $f^{k}(\omega)$ is between two consecutive critical points, $x_{l_{k}}$ and $x_{l_{k}+1}$, and is contained in some $I\left(l_{k}, s_{k}, j_{k}\right)^{+}$with $s_{k} \leq s(\tau)$. Note that by the exponential character of the initial partition, we have

$$
\left|f^{k}(\omega)\right| \leq C\left|I\left(l_{k}, s_{k}, 1\right)^{+}\right| \quad \text { and } \quad \Delta_{k}(\omega) \geq C^{-1}\left|I\left(l_{k}, s_{k}\right)\right|
$$

for some constant $C>0$ depending only on $\hat{f}$ (see Remark 2.1). 
Otherwise for free iterates $y_{k} \in \mathbb{S}^{1} \backslash[-\varepsilon, \varepsilon]$ we get

$$
\begin{aligned}
& \sum_{\substack{r_{i}+p_{i}<k<r_{i+1} \\
\left|y_{k}\right|>\varepsilon}}\left|\frac{f^{\prime}\left(z_{k}\right)-f^{\prime}\left(y_{k}\right)}{f^{\prime}\left(y_{k}\right)}\right| \\
& \leq \frac{L}{\tilde{\sigma}} \sum_{\substack{r_{i}+p_{i}<k<r_{i+1} \\
\left|y_{k}\right|>\varepsilon}}\left|z_{k}-y_{k}\right| \leq \frac{L}{\tilde{\sigma}} \sum_{\substack{r_{i}+p_{i}<k<r_{i+1} \\
\left|y_{k}\right|>\varepsilon}}\left|f^{k}(\omega)\right| \\
& \leq \frac{L}{\tilde{\sigma}} \sum_{r_{i}+p_{i}<k<r_{i+1}} \sigma_{0}^{k-r_{i+1}}\left|f^{r_{i+1}}(\omega)\right| \leq K_{2} \frac{\left|\omega_{i+1}\right|}{\Delta_{r_{i+1}}(\omega)}
\end{aligned}
$$

by definition of $f$ on $\mathbb{S}^{1} \backslash[-\varepsilon, \varepsilon]$, since $\left|f^{\prime}\right| \mathbb{S}^{1} \backslash[-\varepsilon, \varepsilon] \mid>\tilde{\sigma}$ and $\left|f^{\prime \prime}\right| \mathbb{S}^{1} \backslash[-\varepsilon, \varepsilon] \mid$ $\leq L$ for some constant $L$. We also recall that $\Delta_{r_{i+1}}(\omega)<1$ by definition.

For an escape time $k=r_{i}$ with $i \in\{1, \ldots, \gamma\}$ we have either $\left|y_{k}\right| \leq \varepsilon$ and then we have the inequality (4.10), or $\left|y_{k}\right|>\varepsilon$ and we get as in (4.12)

$$
\left|\frac{f^{\prime}\left(z_{k}\right)-f^{\prime}\left(y_{k}\right)}{f^{\prime}\left(y_{k}\right)}\right| \leq \frac{L}{\tilde{\sigma}}\left|z_{k}-y_{k}\right| \leq \frac{L}{\tilde{\sigma}}\left|f^{k}(\omega)\right| \leq K_{3} \frac{\left|\omega_{i}\right|}{\Delta_{r_{i}}(\omega)} .
$$

Hence up to now all cases are bounded by the same type of expression.

Next we find a bound for iterates during binding times. Let us fix $i=$ $1, \ldots, \gamma$ such that $r_{i}$ is not an escape time for $\omega$, i.e. it is either an essential or inessential return time. Then for $k=r_{i}$ we have the same bound (4.10). For $r_{i}<k \leq r_{i}+p_{i}$ we get, for some $\xi \in \omega$,

$$
\begin{aligned}
\left|z_{k}-y_{k}\right| & =\left|\left(f^{k-r_{i}}\right)^{\prime}\left(f^{r_{i}}(\xi)\right)\right| \cdot\left|z_{r_{i}}-y_{r_{i}}\right| \leq\left|\left(f^{k-r_{i}}\right)^{\prime}\left(f^{r_{i}}(\xi)\right)\right| \cdot\left|f^{r_{i}}(\omega)\right| \\
& =\left|\left(f^{k-r_{i}-1}\right)^{\prime}\left(f^{r_{i}+1}(\xi)\right)\right| \cdot\left|f^{\prime}\left(f^{r_{i}}(\xi)\right)-f^{\prime}\left(x_{l_{i}}\right)\right| \cdot\left|\omega_{i}\right| \\
& \leq C\left|\left(f^{k-r_{i}-1}\right)^{\prime}\left(f^{r_{i}+1}(\xi)\right)\right| \cdot\left|f^{\prime \prime}\left(x_{l_{i}}\right)\right| \cdot\left|f^{r_{i}}(\xi)-x_{l_{i}}\right| \cdot\left|\omega_{i}\right|,
\end{aligned}
$$

where we have used the Mean Value Theorem applied to $f^{\prime}$ near the critical point $x_{l_{i}}$ together with the upper bound from Lemma 4.2.

We now have two possibilities by definition of $p_{i}$. On the one hand, for the first case in (3.3) there exists $w \in\left[f\left(x_{l_{i}}\right), f^{r_{i}+1}(\xi)\right]$ such that, by second order Taylor expansion and the lower bound from Lemma 4.2,

$$
\begin{aligned}
\mid f^{k-r_{i}}\left(x_{l_{i}}\right) & -x_{m\left(k-r_{i}-1\right)}\left|e^{-\tau\left(k-r_{i}\right)} \geq\right| f^{k}(\xi)-f^{k-r_{i}}\left(x_{l_{i}}\right) \mid \\
& =\left|\left(f^{k-r_{i}-1}\right)^{\prime}(w)\right| \cdot\left|f^{r_{i}+1}(\xi)-f\left(x_{l_{i}}\right)\right| \\
& \geq C^{-1}\left|\left(f^{k-r_{i}-1}\right)^{\prime}(w)\right| \cdot\left|f^{\prime \prime}\left(x_{l_{i}}\right)\right| \cdot\left|f^{r_{i}}(\xi)-x_{l_{i}}\right|^{2} \\
& \geq(A C)^{-1}\left|\left(f^{k-r_{i}-1}\right)^{\prime}\left(f^{r_{i}+1}(\xi)\right)\right| \cdot\left|f^{\prime \prime}\left(x_{l_{i}}\right)\right| \cdot\left|f^{r_{i}}(\xi)-x_{l_{i}}\right|^{2},
\end{aligned}
$$

where we have used Lemma 4.4 in the last inequality. Note that we can use the lower bound from Lemma 4.2 since $r_{i}$ is an essential or inessential return time for $\omega$. 
The last two expressions together show that

$$
\left|z_{k}-y_{k}\right| \cdot\left|f^{r_{i}}(\xi)-x_{l_{i}}\right| \leq A C^{2}\left|f^{k-r_{i}}\left(x_{l_{i}}\right)-x_{m\left(k-r_{i}-1\right)}\right| e^{-\tau\left(k-r_{i}\right)}\left|\omega_{i}\right| .
$$

This and Lemma 1.4 provide

$$
\begin{aligned}
\left|\frac{f^{\prime}\left(z_{k}\right)-f^{\prime}\left(y_{k}\right)}{f^{\prime}\left(y_{k}\right)}\right| & \leq K_{1}\left|\frac{z_{k}-y_{k}}{y_{k}-\tilde{x}_{k}}\right| \\
& \leq A C^{2} K_{1} e^{-\tau\left(k-r_{i}\right)} \frac{\left|\omega_{i}\right| \cdot\left|f^{k-r_{i}}\left(x_{l_{i}}\right)-x_{m\left(k-r_{i}-1\right)}\right|}{\left|f^{r_{i}}(\xi)-x_{l_{i}}\right| \cdot\left|y_{k}-\tilde{x}_{k}\right|} .
\end{aligned}
$$

To bound the denominator from below, we note that clearly $\left|f^{r_{i}}(\xi)-x_{l_{i}}\right| \geq$ $\Delta_{r_{i}}(\omega)$ since $\xi \in \omega$ and $x_{l_{i}} \in \mathcal{C}$. Moreover, from Proposition 3.4, the closest critical point $\tilde{x}_{k}$ to $y_{k}$ and the closest critical point $x_{m\left(k-r_{i}-1\right)}$ to $f^{k-r_{i}}\left(x_{l_{i}}\right)$ are either equal or else consecutive critical points of $f$, and in the latter case, both $\omega_{k}$ and $f^{k-r_{i}}\left(x_{l_{i}}\right)$ lie between these consecutive critical points. In the case $\tilde{x}_{k}=x_{m\left(k-r_{i}-1\right)}$ we can bound the previous expression by

$$
\begin{aligned}
D e^{-\tau\left(k-r_{i}\right)} \frac{\left|\omega_{i}\right|}{\Delta_{r_{i}}(\omega)} & \cdot \frac{\left|f^{k-r_{i}}\left(x_{l_{i}}\right)-x_{m\left(k-r_{i}-1\right)}\right|}{\left|f^{k-r_{i}}\left(x_{l_{i}}\right)-x_{m\left(k-r_{i}-1\right)}\right|-\left|y_{k}-f^{k-r_{i}}\left(x_{l_{i}}\right)\right|} \\
& \leq D \frac{e^{-\tau\left(k-r_{i}\right)}}{1-e^{-\tau\left(k-r_{i}\right)}} \cdot \frac{\left|\omega_{i}\right|}{\Delta_{r_{i}}(\omega)} \leq D_{1} e^{-\tau\left(k-r_{i}\right)} \frac{\left|\omega_{i}\right|}{\Delta_{r_{i}}(\omega)} .
\end{aligned}
$$

But when $\tilde{x}_{k} \neq x_{m\left(k-r_{i}-1\right)}$, since $y_{k}$ and $f^{k-r_{i}}\left(x_{l_{i}}\right)$ are between these critical points, we have

$$
\begin{aligned}
\left|\tilde{x}_{k}-y_{k}\right| & \geq\left|\tilde{x}_{k}-f^{k-r_{i}}\left(x_{l_{i}}\right)\right|-\left|y_{k}-f^{k-r_{i}}\left(x_{l_{i}}\right)\right| \\
& \geq\left|f^{k-r_{i}}\left(x_{l_{i}}\right)-x_{m\left(k-r_{i}-1\right)}\right|-e^{-\tau\left(k-r_{i}\right)}\left|f^{k-r_{i}}\left(x_{l_{i}}\right)-x_{m\left(k-r_{i}-1\right)}\right| \\
& =\left(1-e^{-\tau\left(k-r_{i}\right)}\right)\left|f^{k-r_{i}}\left(x_{l_{i}}\right)-x_{m\left(k-r_{i}-1\right)}\right|,
\end{aligned}
$$

and we arrive at the same bound as before.

On the other hand, for the second case in (3.3) we get a similar inequality in (4.14) providing

$$
\left|z_{k}-y_{k}\right| \cdot\left|f^{r_{i}}(\xi)-x_{l_{i}}\right| \leq A C^{2} \varepsilon^{1+\tau} e^{-\tau\left(k-r_{i}\right)}\left|\omega_{i}\right|,
$$

and thus by definition of $\tilde{f}$ we get

$$
\begin{aligned}
\left|\frac{f^{\prime}\left(z_{k}\right)-f^{\prime}\left(y_{k}\right)}{f^{\prime}\left(y_{k}\right)}\right| & \leq \frac{L\left|z_{k}-y_{k}\right|}{\tilde{\sigma}} \leq \frac{A C L}{\tilde{\sigma}} e^{-\tau\left(k-r_{i}\right)} \frac{\left|\omega_{i}\right| \varepsilon^{1+\tau}}{\left|f^{r_{i}}(\xi)-x_{l_{i}}\right|} \\
& \leq D_{2} e^{-\tau\left(k-r_{i}\right)} \frac{\left|\omega_{i}\right|}{\Delta_{r_{i}}(\omega)} .
\end{aligned}
$$

This shows that for every $i=1, \ldots, \gamma$ which is not an escape time, we have

$$
\sum_{k=r_{i}}^{r_{i}+\ell}\left|\frac{f^{\prime}\left(z_{k}\right)-f^{\prime}\left(y_{k}\right)}{f^{\prime}\left(y_{k}\right)}\right| \leq D_{3} \frac{\left|\omega_{i}\right|}{\Delta_{r_{i}}(\omega)} \leq \frac{1}{C} \cdot \frac{\left|I\left(l_{i}, s_{i}, j_{i}\right)^{+}\right|}{\left|I\left(l_{i}, s_{i}\right)\right|}
$$


for all $\ell=1, \ldots, p_{i}$, where we have used the definition of $\omega_{i}$ and of host interval, together with the same estimate as in (4.11).

Considering (4.10), (4.12), (4.13) and (4.15) and summing over all iterates we obtain

$$
\begin{aligned}
\sum_{k=0}^{n-1}\left|\frac{f^{\prime}\left(z_{k}\right)-f^{\prime}\left(y_{k}\right)}{f^{\prime}\left(y_{k}\right)}\right| \leq & D_{4} \sum_{k \in F_{1}} \frac{\left|f^{k}(\omega)\right|}{\Delta_{k}(\omega)} \\
& +\left(\frac{L}{\tilde{\sigma}}+K_{2}\right) \sum_{k \in F_{2}}\left|f^{k}(\omega)\right| .
\end{aligned}
$$

Here $F_{1}$ is the set of free iterates together with return situations (essential and inessential returns and escapes) from $k=0$ to $k=n-1$ and $F_{2}$ is the set of free iterates which are not followed by any return, from $r_{\gamma}+p_{\gamma}$ to $n$. Moreover, $D_{4}$ is a constant depending only on $\varepsilon, \tau$ and $\tilde{\sigma}$. So if we can bound (4.16) uniformly we then also find a uniform bound on (4.9) and complete the proof of the lemma.

The righmost sum in (4.16) is bounded:

$$
\sum_{k \in F_{2}}\left|f^{k}(\omega)\right| \leq \sum_{k=0}^{n-1} \tilde{\sigma}^{k-n}\left|f^{n}(\omega)\right| \leq C,
$$

since $\left|f^{n}(\omega)\right|$ is always less than 1 . Now we bound the other sum:

$$
\begin{aligned}
\sum_{k \in F_{1}} \frac{\left|f^{k}(\omega)\right|}{\Delta_{k}(\omega)} & \leq \sum_{\substack{k \in F_{1} \\
\left(l_{k}, s_{k}\right)=(0,0)}} \frac{\left|f^{k}(\omega)\right|}{\Delta_{k}(\omega)}+\sum_{|l| \geq k_{0}|s| \geq 1} \sum_{\substack{k \in F_{1} \\
\left(l_{k}, s_{k}\right)=(l, s)}} \frac{\left|f^{k}(\omega)\right|}{\Delta_{k}(\omega)} \\
& \leq C \frac{\sigma_{1}}{\sigma_{1}-1}+\sum_{|l| \geq k_{0}} \sum_{|s| \geq 1} \frac{\sigma_{1}}{\sigma_{1}-1} \cdot \frac{1}{C} \cdot \frac{\left|f^{q(l, s)}(\omega)\right|}{|I(l, s)|}
\end{aligned}
$$

by (4.11), where $q(l, s)=\max \left\{0 \leq k \leq n-1:\left(\hat{l}_{k}, \hat{s}_{k}\right)=(l, s)\right\}$ and we make the convention that whenever $\left\{0 \leq k \leq n-1:\left(\hat{l}_{q}, \hat{s}_{q}\right)=(l, s)\right\}=\emptyset$ we have $\left|f^{q(l, s)}(\omega)\right| /|I(l, s)|=0$. We have used the following estimate for any given fixed value of $(l, s)$ :

$$
\begin{aligned}
\sum_{\left\{k: \hat{s}_{k}=s\right\}}\left|f^{k}(\omega)\right| & \leq\left|f^{q(l, s)}(\omega)\right| \sum_{\left\{k:\left(\hat{l}_{k}, \hat{s}_{k}\right)=(l, s)\right\}} \sigma_{1}^{k-q(l, s)} \leq \frac{\sigma_{1}}{\sigma_{1}-1}\left|f^{q(l, s)}(\omega)\right| \\
& \leq C\left|I(l, s, j)^{+}\right|,
\end{aligned}
$$

because writing $\left\{k: \hat{s}_{k}=s\right\}=\left\{k_{1}<\cdots<k_{h}\right\}$ we have $\left|f^{k_{i}}(\omega)\right| \leq$ $\sigma_{1}^{-1}\left|f^{k_{i+1}}(\omega)\right|$ for $i=1, \ldots, h$, where $1<\sigma_{1}=\min \left\{\sigma_{0}, e^{(1-2 \zeta)(\pi / \beta)\left(k_{0}+1\right)}\right\} \leq$ $\min \left\{\sigma_{0}, e^{(1-2 \zeta)(\pi / \beta)(|l|+|s|)}\right\}$, by Lemma 4.6(b) together with Remark 3.3. 
We observe that by construction we must have $\left|I(l, s, j)^{+}\right| /|I(l, s)| \leq$ $9(|l|+|s|)^{-3}$ and so we arrive at

$$
\sum_{k \in F_{1}} \frac{\left|f^{k}(\omega)\right|}{\Delta_{k}(\omega)} \leq C \sum_{|l| \geq k_{0}} \sum_{|s| \geq 1} \frac{9}{(|l|+|s|)^{3}}<\infty,
$$

finishing the proof of the lemma.

5. Probability of deep essential returns. Here we use the results from Section 4 to estimate the probability of having an orbit with a given sequence of host intervals at essential return situations.

Recall that $\mathcal{C}_{\infty}=\bigcup_{n=0}^{\infty}\left(f^{n}\right)^{-1}(\mathcal{C})$ is the set of pre-orbits of the critical set $\mathcal{C}$. For each $x \in I \backslash \mathcal{C}_{\infty}$ let $\omega$ be the element of $\mathcal{P}_{n}$ which contains $x$.

Consider the sets $R_{n}(\omega)$ and $Q_{n}(\omega)$ of return situations (essential and inessential returns and escapes) and indices of host intervals for $\omega$, during the iterates 0 to $n$. Let $u_{n}(\omega)$ denote, for $\omega \in \mathcal{P}_{n}$, the number of essential returns or escapes associated to $\omega$ between 0 and $n$, let $0 \leq t_{1}(\omega) \leq \cdots \leq t_{u_{n}}(\omega) \leq n$ be the instants of occurrence of the essential returns or escapes, and let $\left(l_{1}, s_{1}, j_{1}\right), \ldots,\left(l_{u_{n}}, s_{u_{n}}, j_{u_{n}}\right)$ be the corresponding critical points and indices of the respective host intervals. We say that the sum $\left|s_{i}\right|+\left|j_{i}\right|$ is the depth of the corresponding return of $\omega$.

Note that by construction $t_{1}(\omega)=0$ for all $\omega \in \mathcal{P}_{0} \backslash I(0,0,0)$ and $t_{1}(\omega)$ $=1$ for $\omega=I(0,0,0)$ (see Remark 3.1).

Lemma 5.1 (No return probability). For every $n \geq 0$ there exists no non-degenerate interval $\omega \in \mathcal{P}_{n}$ such that $\omega \in \mathcal{P}_{n+k}$ for all $k \geq 1$. Moreover, there exist constants $0<\xi_{0}<1$ and $K_{0}>0$ (depending only on $\sigma, \sigma_{0}$ and on $\zeta$ from Lemma 4.6), and $n_{0} \geq 1$ such that for every $n>n_{0}$ we have $\lambda\left(\bigcup\left\{\omega \in \mathcal{P}_{n}: u_{n} \mid \omega=1\right\}\right) \leq K_{0} e^{-\xi_{0} n}$.

Proof. If $\omega \in \mathcal{P}_{n+k}$ for all $k \geq 0$, then $\omega$ is not refined in all future iterates. This means that $f^{n+k}(\omega)$ has no essential returns nor escapes for $k \geq 1$. Hence every iterate is either free or a binding time associated to an inessential return. Let $p_{0}, p_{1}, p_{2}, \ldots$ be the lengths of the binding periods associated to inessential return times (if any) $t<r_{0}<r_{1}<r_{2}<\cdots$ for $\omega$ after $t$, where $0 \leq t \leq n$ is the last essential return time or escape before $n$. Let $k \geq 0$ and $r_{i}+p_{i} \leq n+k<r_{i+1}$ for some $i \geq 0$, where we set $r_{i+1}=\infty$ if $r_{i}$ is the last inessential return time after $t$ (it may happen that $r_{1}=\infty$, in which case $i=0$ and there are no inessential returns after $t$ ). Lemmas 4.6 and 4.8 ensure that

$$
2 \geq\left|f^{n+k}(\omega)\right| \geq 2^{i} \sigma_{0}^{n+k-t-\sum_{j=0}^{i}\left(p_{j}+1\right)}\left|f^{t}(\omega)\right|,
$$

for arbitrarily large values of $k \geq 0$, where the exponent of $\sigma_{0}$ counts the number of free iterates between time $t$ and $n+k$. 
Note that if there are no inessential returns, then $i=0$ and so

$$
\left|f^{t}(\omega)\right| \leq 2 \sigma_{0}^{t+p_{0}+1-n-k} .
$$

Otherwise $\left|f^{t}(\omega)\right| \leq 2^{1-i} \sigma_{0}^{t+\sum_{j=0}^{i}\left(p_{j}+1\right)-n-k}$ from (5.1).

We conclude that $\left|f^{t}(\omega)\right|=0$, which is not possible for a non-degenerate interval. This proves the first part of the lemma.

Now let $\omega \in \mathcal{P}_{n}$ be such that $u_{n}(\omega)=1$. Then by the construction of the refinement, we see that $\omega \in \mathcal{P}_{i}$ for all $0 \leq i \leq n$. Hence either

- $\omega=I(0,0,0)$ with $t_{1}=1$ the unique essential return up to iterate $n$ and $f(\omega)=I(l, s, j)$ with $(l, s, j) \neq(0,0,0)$; or

- $\omega=I(l, s, j)$ with $|l| \geq k_{0},|s| \geq 1$ and $j=1, \ldots,(|l|+|s|)^{3}$, having a single essential return $t_{1}=0$ up to iterate $n$.

We concentrate on the latter case and write $p_{0}, p_{1}, p_{2}, \ldots$ and $0=t_{1}=r_{0}<$ $r_{1}<r_{2}<\cdots$ for the binding periods associated to their respective inessential return times of the orbit of $\omega$ as before. Then by (5.1) and (5.2), and since during binding periods the intervals of the partition are not subdivided (see Section 3), we have either $n \leq r_{0}+p_{0}=p_{0}$ or

$$
\begin{aligned}
|\omega| \leq 2 \sigma_{0}^{p_{0}-n} & \text { if } p_{0}(\omega)<n \leq r_{1}(\omega)+p_{1}(\omega) \leq \infty, \quad \text { or } \\
|\omega| \leq 2^{1-i} \sigma_{0}^{-\left(n-\sum_{j=0}^{i}\left(p_{j}(\omega)+1\right)\right)} & \text { for } i \geq 1 \text { such that } \\
& r_{i}(\omega)+p_{i}(\omega)<n \leq r_{i+1}(\omega)+p_{i+1}(\omega) .
\end{aligned}
$$

We note that in the case of $f(\omega)=I(l, s, j)$, that is, when $t_{1}(\omega)=1$, we can repeat the arguments for the interval $f(\omega)$, arriving at the same bounds for $|\omega|$ except for an extra factor of $\tilde{\sigma}$ since $|f(\omega)| \geq \tilde{\sigma}|\omega|$. Hence we may write, according to the three cases above,

$$
\begin{aligned}
\lambda\left(\cup\left\{\omega \in \mathcal{P}_{n}: u_{n} \mid \omega=1\right\}\right) \leq & \sum_{n \leq p_{0}(\omega)}|\omega|+\sum_{p_{0}(\omega)<n \leq r_{1}(\omega)+p_{1}(\omega) \leq \infty}|\omega| \\
& +\sum_{\substack{r_{i}(\omega)+p_{i}(\omega)<n \leq r_{i+1}(\omega)+p_{i+1}(\omega) \\
=}}|\omega|+S_{3}+S_{1}+S_{2}+S_{3},
\end{aligned}
$$

where, by the above comment, we may assume that every sum ranges over $\omega \in \mathcal{P}_{0} \backslash I(0,0,0)$ and $S_{3}$ corresponds to the sum over the partition elements in $I(0,0,0) \cap \mathcal{P}_{1}$, which is bounded by $\left(S_{0}+S_{1}+S_{2}\right) / \tilde{\sigma}$.

For $S_{0}$ we use Lemma 4.6(a) to deduce that the summands in $S_{0}$ are the elements of $\mathcal{P}_{0}$ such that $n \leq p_{0}(\omega) \leq \frac{2 \pi}{\beta \log \sigma}(|l|+|s|)$, that is, $|l|+|s| \geq$ $\frac{\beta \log \sigma}{2 \pi} n$, thus by Remark 2.1, setting $C_{0}=\frac{\beta \log \sigma}{2 \pi}$, we have 


$$
\begin{aligned}
S_{0} & \leq \sum_{|l|+|s| \geq C_{0} n} a_{1} \frac{e^{-(\pi / \beta)(|l|+|s|)}}{(|l|+|s|)^{3}} \leq a_{1} \sum_{k \geq C_{0} n} k \frac{e^{-(\pi / \beta) k}}{k^{3}} \\
& \leq \frac{a_{1}}{\left(C_{0} n\right)^{2}} \cdot \frac{e^{-(\pi / \beta) C_{0} n}}{1-e^{-C_{0} n}} \leq K_{0}^{\prime} \sigma^{-n / 2} .
\end{aligned}
$$

We write $S_{1}=S_{11}+S_{12}$ where

$$
S_{11}=\sum_{p_{0}(\omega) \leq n / 2}|\omega| \text { and } S_{12}=\sum_{n / 2<p_{0}(\omega)}|\omega| \leq K_{0}^{\prime} \sigma^{-n / 4}
$$

and we have used the bound (5.3). We also split $S_{2}=S_{21}+S_{22}$ according to whether $n-\sum_{k=0}^{i}\left(p_{k}(\omega)+1\right) \geq n / 2$ or not, obtaining

$$
S_{21}=\sum_{|\omega| \leq 2^{1-i} \sigma_{0}^{-n / 2}}|\omega| \text { and } S_{22}=\sum_{n-\sum_{j=0}^{i}\left(p_{j}(\omega)+1\right)<n / 2}|\omega| .
$$

Since $2^{1-i} \leq 2$ we get $S_{11}+S_{21} \leq 2 S_{11}$ and the summands $\omega \in \mathcal{P}_{0}$ satisfy $|\omega| \leq 2 \sigma_{0}^{-n / 2}$, thus by Remark 2.1 we get

$$
|l|+|s| \geq \frac{n}{2} \cdot \frac{\log \sigma_{0}}{3+\pi / \beta}+\frac{-\log \left(2 / a_{1}\right)}{3+\pi / \beta} \geq C_{1} \frac{n}{4},
$$

where $C_{1}=\log \sigma_{0} /(3+\pi / \beta)$ for every large enough $n$. Then $S_{11}+S_{21} \leq$ $2 K_{0}^{\prime \prime} \sigma_{0}^{-n / 5}$ by the same calculations as in (5.3) with slightly different constants.

For $S_{22}$ we note that $n-\sum_{k=0}^{i}\left(1+p_{k}(\omega)\right)<n / 2$ implies $\sum_{k=0}^{i}\left(1+p_{k}(\omega)\right)$ $>n / 2$, and so by Lemma $4.6(\mathrm{~b})$ we get

$$
2 \geq\left|f^{r_{i}+p_{i}+1}(\omega)\right|>e^{(1-2 \zeta)(\pi / \beta) \sum_{j=0}^{i}\left(1+p_{j}(\omega)\right)}|\omega|>e^{n \pi(1-2 \zeta) /(2 \beta)}|\omega|
$$

and hence again by Remark 2.1, for every large enough $n$, we must have $|l|+|s|>C_{2} n / 4$ where $C_{2}=\pi(1-2 \zeta) /(3 \beta+\pi)$. We deduce that $S_{22} \leq$ $K_{0}^{\prime \prime \prime} e^{-(\pi / \beta) C_{2} n / 4}$ following the same calculations as in (5.3).

Putting all together we see that there are constants $0<\xi_{0}<1, K_{0}>0$ and $n_{0} \geq 1$ such that $S_{0}+S_{1}+S_{2}+S_{3} \leq K_{0} e^{-\xi_{0} n}$ for all $n \geq n_{0}$, with $\xi_{0}$ and $K_{0}$ depending on $\sigma, \sigma_{0}$ and $\zeta$, as stated.

Let $v \leq u \leq n$ and let $v$ pairs $\left(\eta_{1}, v_{1}\right), \ldots,\left(\eta_{v}, v_{v}\right)$ of positive integers be given, with $\eta_{i} \geq k_{0}, v_{i} \geq 1$ and $\eta_{i}+v_{i} \geq \Theta$, where

$$
\Theta=\Theta(b)=\frac{\beta}{\pi} \log \frac{a_{2}}{b} .
$$

This value of $\Theta$ is chosen so as to have $\operatorname{dist}(I(\eta, v), \mathcal{C})<b$ if, and only if, $\eta+v \geq \Theta$. We assume that $b$ is so small that $\Theta \geq \theta$ (recall that $\theta$ was defined in Lemma 4.6) and, following Remark 4.9, that $\Theta$ is large enough in order that $Q(l, s, \tau, \rho)>1$ for all $|l|+|s| \geq \Theta$. 
For $\omega \in \mathcal{P}_{n}$ with $u_{n}(\omega)=u$, let $0=t_{1}<\cdots<t_{u} \leq n$ be the return situations (essential returns or escapes) of $\omega,\left(l_{i}, s_{i}\right)$ the corresponding indices of the host intervals, and $d_{n}(\omega)=v$ the number of pairs $\left(l_{i}, s_{i}\right)$ such that $\left|l_{i}\right|+\left|s_{i}\right| \geq \Theta$. We say that a return situation whose depth satisfies $\left|l_{i}\right|+\left|s_{i}\right| \geq \Theta$ is a deep return. Denote by $1 \leq r_{1}<\cdots<r_{v} \leq u$ the indices of the return situations corresponding to deep returns.

We now define the subset $A_{\left(\eta_{1}, v_{1}\right), \ldots,\left(\eta_{v}, v_{v}\right)}^{u, v}(n)$ as

$$
\bigcup\left\{\omega \in \mathcal{P}_{n}: u_{n}(\omega)=u, d_{n}(\omega)=v \text { and }\left|f^{t_{r_{i}}}(\omega)\right| \subset I\left(\eta_{i}, v_{i}\right), i=1, \ldots, v\right\} .
$$

Proposition 5.2 (Probability of essential returns with specified depths). If $\Theta$ is large enough (depending on $D_{0}$ from Lemma 4.10), then for every large $n \geq u \geq v \geq 1$ and $\vartheta=\max \{\alpha, 3 \zeta\}$,

$$
\lambda\left(A_{\left(\eta_{1}, v_{1}\right), \ldots,\left(\eta_{v}, v_{v}\right)}^{u, v}(n)\right) \leq\left(\begin{array}{l}
u \\
v
\end{array}\right) \exp \left[(2 \vartheta-1) \frac{\pi}{\beta} \sum_{i=1}^{v}\left(\eta_{i}+v_{i}\right)\right] .
$$

Proof. We start by fixing $n \in \mathbb{N}, u \in\{1, \ldots, n\}, v \in\{1, \ldots, u\}$ and taking $\omega^{0} \in \mathcal{P}_{0}$. Let $\omega \in \mathcal{P}_{n}$ be such that $\omega \subset \omega^{0}$ and $u_{n}(\omega)=u$ and $1=t_{1}<\cdots<t_{u} \leq n$ be the return situations (essential returns or escapes) of $\omega$.

For $m=1, \ldots, u$ we write $\omega^{m}=\omega\left(\left(l_{1}, s_{1}, j_{1}\right), \ldots,\left(l_{m}, s_{m}, j_{m}\right)\right) \in \mathcal{P}_{t_{m}}$ for the subset of $\omega^{0}$ satisfying

$$
\begin{aligned}
& f^{t_{i}}\left(\omega^{m}\right) \subset I\left(l_{i}, s_{i}, j_{i}\right)^{+}, \quad i \in\{1, \ldots, m-1\}, \\
& I\left(l_{m}, s_{m}, j_{m}\right) \subset f^{t_{m}}\left(\omega^{m}\right) \subset I\left(l_{m}, s_{m}, j_{m}\right)^{+},
\end{aligned}
$$

by the definition of the sequence of partitions $\mathcal{P}_{n}$. Note that we get a nested sequence of sets

$$
\omega^{0} \supsetneq \omega^{1} \supsetneq \cdots \supsetneq \omega^{u}=\omega .
$$

We define $\mathcal{T}=\left\{\omega \in \mathcal{P}_{n}: \omega \subset \omega^{0}, u_{n}(\omega)=u\right\}$ and consider the sequence of deep return situations (essential or escapes) of each $\omega \in \mathcal{T}: 1 \leq r_{1}<\cdots<$ $r_{v} \leq u$, that is, the indices of the return situations such that for $i=1, \ldots, v$,

$$
\left|f^{t_{r_{i}}}(x)\right| \in I\left(\eta_{i}, v_{i}\right) \quad \text { for all } x \in \omega \text { and } \eta_{i}+v_{i} \geq \Theta \text {. }
$$

Now we define by induction a sequence of partitions of $\mathcal{T}$ which will enable us to determine the estimates we need.

Start by putting $\mathcal{V}_{0}=\bigcup\{\omega \in \mathcal{T}\}$. For $1 \leq i \leq v$ and $h \geq 0$ such that $r_{i} \leq r_{i}+h<r_{i+1}$ we define the subset

$$
\begin{aligned}
& \mathcal{V}_{r_{i}+h}^{j^{1}, \ldots, j^{i}}=\bigcup\left\{\omega^{r_{i}+h}: \omega \in \mathcal{T} \&(5.5) \text { holds with } m=r_{i}+h\right. \\
& \left.\qquad \& j^{k}=j_{r_{k}} \text { for } k=1, \ldots, i\right\},
\end{aligned}
$$

where we make the convention $r_{v+1}=u$; and for $1 \leq h<r_{1}$ we set $\mathcal{V}_{h}=$ $\bigcup\left\{\omega^{h}: \omega \in \mathcal{T}\right\}$. 
Now we compare $\lambda\left(\mathcal{V}_{r_{i}}^{j^{1}, \ldots, j^{i}}\right)$ with $\lambda\left(\mathcal{V}_{r_{i-1}}^{j^{1}, \ldots, j^{i-1}}\right)$. We claim that

$$
\lambda\left(\mathcal{V}_{r_{i}}^{j^{1}, \ldots, j^{i}}\right) \leq \frac{C}{\left(\eta_{i}+v_{i}\right)^{3}} \cdot \frac{e^{-(\pi / \beta)\left(\eta_{i}+v_{i}\right)}}{e^{-\vartheta(\pi / \beta)\left(\eta_{i-1}+v_{i-1}\right)}} \lambda\left(\mathcal{V}_{r_{i-1}}^{j^{1}, \ldots, j^{i-1}}\right),
$$

where $\vartheta=\max \{2 \zeta, \alpha\} \in(0,1)$. Assuming this claim we deduce

$$
\begin{gathered}
\lambda\left(\mathcal{V}_{u}^{j^{1}, \ldots, j^{v}}\right) \leq \cdots \leq \lambda\left(\mathcal{V}_{v}^{j^{1}, \ldots, j^{v}}\right) \leq \frac{C}{\left(\eta_{v}+v_{v}\right)^{3}} \cdot \frac{e^{-(\pi / \beta)\left(\eta_{v}+v_{v}\right)}}{e^{-\vartheta(\pi / \beta)\left(\eta_{v-1}+v_{v-1}\right)} \lambda\left(\mathcal{V}_{r_{v}-1}^{j^{1}, \ldots, j^{v-1}}\right)} \\
\quad \leq\left(\prod_{i=1}^{s} \frac{C}{\left(\eta_{i}+v_{i}\right)^{3}}\right) \exp \left(-\frac{\pi}{\beta} \sum_{i=1}^{v}\left(\eta_{i}+v_{i}\right)+\vartheta \frac{\pi}{\beta} \sum_{i=1}^{v}\left(\eta_{i-1}+v_{i-1}\right)\right) \lambda\left(\mathcal{V}_{0}\right) .
\end{gathered}
$$

Finally, we need to consider all possible combinations of the events $\mathcal{V}_{u}^{j^{1}, \ldots, j^{v}}$ which are included in $A_{\left(\eta_{1}, v_{1}\right), \ldots,\left(\eta_{v}, v_{v}\right)}^{u, v}(n)$. Note that for any given $v \leq u$ there are $\left(\begin{array}{l}u \\ v\end{array}\right)$ ways of having $v$ deep returns among $u$ return situations and, by symmetry, for any sequence of deep returns with given depth $\left(\eta_{i}, v_{i}\right)$ there are $4\left(\eta_{i}+v_{i}\right)^{3}$ different possibilities of falling in an element of the partition $\mathcal{P}_{0}$. Thus since $\lambda\left(\mathcal{V}_{0}\right) \leq \lambda\left(\omega_{0}\right)$ we arrive at

$$
\begin{aligned}
\lambda\left(A_{\left(\eta_{1}, v_{1}\right), \ldots,\left(\eta_{v}, v_{v}\right)}^{u, v}(n)\right) \leq & \left(\begin{array}{l}
u \\
v
\end{array}\right) \prod_{i=1}^{v} 4\left(\eta_{i}+v_{i}\right)^{3}\left(\prod_{i=1}^{v} \frac{C}{\left(\eta_{i}+v_{i}\right)^{3}}\right) \\
& \cdot \exp \left((\vartheta-1) \frac{\pi}{\beta} \sum_{i=1}^{v}\left(\eta_{i}+v_{i}\right)\right) \sum_{\omega_{0} \in \mathcal{P}_{0}} e^{\vartheta(\pi / \beta)\left(\eta_{0}+v_{0}\right)} \lambda\left(\omega_{0}\right) \\
& \leq\left(\begin{array}{l}
u \\
v
\end{array}\right) \exp \left((2 \vartheta-1) \frac{\pi}{\beta} \sum_{i=1}^{v}\left(\eta_{i}+v_{i}\right)\right),
\end{aligned}
$$

where we have used the fact that $\sum_{\omega_{0} \in \mathcal{P}_{0}} e^{\vartheta(\pi / \beta)\left(\eta_{0}+v_{0}\right)} \lambda\left(\omega_{0}\right)<\infty$ and also that $\sum_{i=1}^{v}\left(\eta_{i}+v_{i}\right) \geq v \Theta$ and that $\Theta$ can be taken as large as needed.

Thus to complete proof it is enough to prove the claim (5.6). For this we proceed as follows.

Given $\omega \in \mathcal{T}$ and $\omega^{r_{i-1}} \in \mathcal{V}_{r_{i-1}}^{j^{1}, \ldots, j^{i-1}}$ we have

$$
\omega^{r_{i}}=\omega^{r_{i-1}} \cap \mathcal{V}_{r_{i}}^{j^{1}, \ldots, j^{i}},
$$

which is the set of points in $\omega^{r_{i-1}}$ which remain in the next level of the partition. We divide the argument into the following cases.

(1) $t_{r_{i}-1}$ is an essential return with depth $(l, s)$.

In this case, since $\omega^{r_{i}} \subsetneq \omega^{r_{i}-1} \subsetneq \cdots \subsetneq \omega^{r_{i-1}+1} \subsetneq \omega^{r_{i-1}}$ and $\omega^{r_{i}} \in \mathcal{P}_{t_{r_{i}}-1}$ by the refinement algorithm, we can use the Bounded Distortion Lemma 4.10 
to write

$$
\begin{aligned}
\frac{\left|\omega^{r_{i}}\right|}{\left|\omega^{r_{i-1} \mid}\right|} & \leq \frac{\left|\omega^{r_{i-1}+1}\right|}{\left|\omega^{r_{i-1}}\right|} \cdots \frac{\left|\omega^{r_{i}}\right|}{\left|\omega^{r_{i}-1}\right|} \leq 1 \cdot 1 \cdots D_{0} \frac{\left|f^{t_{r_{i}}}\left(\omega^{r_{i}}\right)\right|}{\mid f^{t_{r_{i}}\left(\omega^{r_{i}-1}\right) \mid}} \\
& \leq D_{0} \frac{\left|I\left(\eta_{i}, v_{i}, j_{i}\right)^{+}\right|}{a_{1} Q e^{-2 \zeta(\pi / \beta)(|l|+|s|)}} \leq D_{0} \frac{9 a_{1} e^{-(\pi / \beta)\left(\eta_{i}+v_{i}\right)}}{a_{1} Q\left(\eta_{i}+v_{i}\right)^{3} e^{-2 \zeta(\pi / \beta)(|l|+|s|)}} \\
& =\frac{C}{\left(\eta_{i}+v_{i}\right)^{3}} \cdot \frac{e^{-(\pi / \beta)\left(\eta_{i}+v_{i}\right)}}{e^{-2 \zeta(\pi / \beta)(|l|+|s|)}} \leq \frac{C}{\left(\eta_{i}+v_{i}\right)^{3}} \cdot \frac{e^{-(\pi / \beta)\left(\eta_{i}+v_{i}\right)}}{e^{-2 \zeta(\pi / \beta)\left(\eta_{i-1}+v_{i-1}\right)}},
\end{aligned}
$$

where $C=C(\rho, \tau, \varepsilon, \sigma)$ and in the second and third inequalities we used Remark 2.1 and Lemma 4.8(2). In the last inequality we argue as follows.

If $r_{i}=r_{i-1}+1$, then $|l|+|s|=\eta_{i-1}+v_{i-1}$ by definition. If $r_{i}>r_{i-1}+1$, then by definition of deep returns, $|l|+|s|<\Theta \leq \eta_{i-1}+v_{i-1}$.

(2) $t_{r_{1}-1}$ is an escape time having host interval $I(l, s, j)^{+}$with $(l, s, j) \neq$ $\left( \pm k_{0}, 1,1\right)$.

Arguing as in the previous case, we only need to get a lower bound for $\left|f^{t_{r_{i}}}\left(\omega^{r_{i}-1}\right)\right|$. By the Mean Value Theorem, for some $\xi \in f^{t_{r_{i-1}}}\left(\omega^{r_{i}-1}\right)$ we have

$$
\begin{aligned}
\mid f^{t_{r_{i}}\left(\omega^{r_{i}-1}\right) \mid} & =\left|\left(f^{t_{r_{i}}-t_{r_{i}-1}}\right)^{\prime}(\xi)\right| \cdot\left|f^{t_{r_{i}-1}}\left(\omega^{r_{i}-1}\right)\right| \\
& =\left|\left(f^{t_{r_{i}}-t_{r_{i}-1}-1}\right)^{\prime}(f(\xi))\right| \cdot\left|f^{\prime}(\xi)\right| \cdot\left|f^{t_{r_{i}-1}}\left(\omega^{r_{i}-1}\right)\right| \\
& \geq \sigma_{0}^{t_{r_{i}}-t_{r_{i}-1}-1} \frac{\tau \hat{x}^{\alpha-1}}{C} e^{(1-\alpha)(\pi / \beta)|l|} \mid f^{t_{r_{i}-1}\left(\omega^{r_{i}-1}\right) \mid} \\
& \geq C(\tau) e^{(1-\alpha)(\pi / \beta)|l|} a_{1} \frac{e^{-(\pi / \beta)(|l|+|s|)}}{(|l|+|s|)^{3}} \\
& =C(\tau) \frac{e^{-(\pi / \beta)(\alpha|l|+|s|)}}{(|l|+|s|)^{3}}=C(\tau) e^{(\alpha-1)(\pi / \beta)|s|} \frac{e^{-\alpha(\pi / \beta)(|l|+|s|)}}{(|l|+|s|)^{3}} \\
& \geq C(\tau) e^{(\alpha-1)(\pi / \beta) s(\tau)} \frac{e^{-\alpha(\pi / \beta)(|l|+|s|)}}{(|l|+|s|)^{3}} \geq C(\tau) e^{-\alpha(\pi / \beta)(|l|+|s|)},
\end{aligned}
$$

where we have used the bound (3.4) in the first inequality and that $|s| \leq s(\tau)$ in the last inequalities. Thus we arrive at a similar bound

$$
\frac{\left|\omega^{r_{i}}\right|}{\left|\omega^{r_{i-1}}\right|} \leq \frac{C}{\left(\eta_{i}+v_{i}\right)^{3}} \cdot \frac{e^{-(\pi / \beta)\left(\eta_{i}+v_{i}\right)}}{e^{-\alpha(\pi / \beta)\left(\eta_{i-1}+v_{i-1}\right)}} .
$$

(3) $t_{r_{i}-1}$ is an escape time having host interval $I\left(k_{0}, 1,1\right)$ or $I\left(-k_{0}, 1,1\right)$.

From Lemma 4.3 , points in $f^{t_{r_{i}-1}}\left(\omega^{r_{i}-1}\right)$ remain outside $[-\varepsilon, \varepsilon]$ during a minimum number $m_{0} \geq \log (1 / \varepsilon)$ of iterates with derivative greater than $\sigma_{0}$, 
which can be taken larger than $e$. Hence

$$
\begin{aligned}
\left|f^{t_{r_{i}}}\left(\omega^{r_{i}-1}\right)\right| & =\left|\left(f^{t_{r_{i}}-t_{r_{i}-1}}\right)^{\prime}(\xi)\right| \cdot\left|f^{t_{r_{i}-1}}\left(\omega^{r_{i}-1}\right)\right| \\
& \geq \sigma_{0}^{m_{0}}\left|f^{t_{r_{i}-1}}\left(\omega^{r_{i}-1}\right)\right| \geq e^{\log (1 / \varepsilon)} \frac{e^{-(\pi / \beta)\left(k_{0}+1\right)}}{\left(k_{0}+1\right)^{3}} \\
& \geq C \frac{e^{\log (1 / \varepsilon)}}{(\log (1 / \varepsilon))^{3}} e^{-(\pi / \beta)\left(k_{0}+1\right)} \geq C e^{-\alpha(\pi / \beta)\left(k_{0}+1\right)},
\end{aligned}
$$

where we have used the fact that $k_{0} \leq C \log (1 / \varepsilon)$ and assumed that $\varepsilon$ is small enough so that $e^{\log (1 / \varepsilon)} \geq(\log (1 / \varepsilon))^{3}$. Thus we arrive again at a bound of the form (5.7). We remark that since $\left|f^{t_{r_{i}}}\left(\omega^{r_{i}-1}\right)\right| \leq 2$ the number of free iterates $t_{r_{i}}-t_{r_{i}-1}$ is bounded from above by a constant depending on $k_{0}, \beta$ and $\sigma_{0}$ only, so that the lower bound we get is uniformly away from zero for all intervals satisfying this third case.

Now we are ready to obtain (5.6) as follows for $1 \leq i \leq v$ :

$$
\begin{aligned}
\lambda\left(\mathcal{V}_{r_{i}}^{j^{1}, \ldots, j^{i}}\right) & =\sum_{\omega^{r_{i-1}} \in \mathcal{V}_{r_{i-1}}^{j^{1}, \ldots, j^{i-1}}} \frac{\left|\omega^{r_{i}}\right|}{\left|\omega^{r_{i-1}}\right|}\left|\omega^{r_{i-1}}\right| \\
& \leq \frac{C}{\left(\eta_{i}+v_{i}\right)^{3}} \cdot \frac{e^{-(\pi / \beta)\left(\eta_{i}+v_{i}\right)}}{e^{-\vartheta(\pi / \beta)\left(\eta_{i-1}+v_{i-1}\right)}} \cdot \lambda\left(\mathcal{V}_{r_{i-1}}^{j^{1}, \ldots, j^{i-1}}\right),
\end{aligned}
$$

where $\vartheta=\max \{2 \zeta, \alpha\} \in(0,1)$ is obtained comparing the bounds for each case.

Using the same notations as before with $\eta \geq k_{0}, \varsigma \geq 1$ and $\eta+\varsigma \geq \Theta$ we define

$$
\begin{aligned}
& A_{(\eta, \varsigma), j}^{v, u}(n)=\bigcup\left\{\omega \in \mathcal{P}_{n}:\left(u_{n}(\omega), d_{n}(\omega)\right)=(u, v)\right. \text { and } \\
& \left.\left|f^{t_{r_{j}}}(x)\right| \in I(\eta, \varsigma), \forall x \in \omega\right\}, \\
& A_{(\eta, \varsigma)}^{v, u}(n)=\bigcup\left\{\omega \in \mathcal{P}_{n}:\left(u_{n}(\omega), d_{n}(\omega)\right)=(u, v)\right. \text { and } \\
& \text { there exists } \left.1 \leq j \leq v \text { such that }\left|f^{t_{r_{j}}}(x)\right| \in I(\eta, \varsigma), \forall x \in \omega\right\} \text {, } \\
& A_{(\eta, \varsigma)}(n)=\bigcup\left\{\omega \in \mathcal{P}_{n} \text { : there exists } t \leq n\right. \text { such that } \\
& \left.t(\omega) \text { is an essential return and }\left|f^{t}(x)\right| \in I(\eta, \varsigma), \forall x \in \omega\right\},
\end{aligned}
$$

and derive the following corollary which will be used during the final arguments.

Corollary 5.3. For $1 \leq j \leq v \leq u \leq n$ we have
(1) $\lambda\left(A_{(\eta, \varsigma), j}^{v, u}(n)\right) \leq\left(\begin{array}{l}u \\ v\end{array}\right) e^{(2 \vartheta-1)(\pi / \beta)(\eta+\varsigma)}$,
(2) $\lambda\left(A_{(\eta, \varsigma)}^{v, u}(n)\right) \leq v\left(\begin{array}{c}u \\ v\end{array}\right) e^{(2 \vartheta-1)(\pi / \beta)(\eta+\varsigma)}$,
(3) $\lambda\left(A_{(\eta, \varsigma)}(n)\right) \leq n^{3} e^{o(\Theta) n} e^{(4 \vartheta-1)(\pi / \beta)(\eta+\varsigma)}$, 
if $\Theta$ is sufficiently large and $\zeta>\vartheta / 3$ is small enough, where $o(\Theta) \rightarrow 0$ as $\Theta \rightarrow \infty$.

Proof. For item (1) we note that since

$$
A_{(\eta, \varsigma), j}^{v, u}(n) \subseteq \bigcup_{\eta_{i}+\varsigma_{i} \geq \Theta, \eta_{i} \geq k_{0}, \varsigma_{i} \geq 1, i \neq j} A_{\left(\eta_{1}, \varsigma_{1}\right), \ldots,\left(\eta_{i-1}, \varsigma_{i-1}\right),(\eta, \varsigma),\left(\eta_{i+1}, \varsigma_{i+1}\right), \ldots,\left(\eta_{u}, \varsigma_{u}\right)}^{v, u}(n)
$$

then

$$
\begin{aligned}
\lambda\left(A_{(\eta, \varsigma), j}^{v, u}(n)\right) & \leq\left(\begin{array}{l}
u \\
v
\end{array}\right)\left(\sum_{l+s \geq \Theta} e^{(2 \vartheta-1)(\pi / \beta)(l+s)}\right)^{v-1} e^{(2 \vartheta-1)(\pi / \beta)(\eta+\varsigma)} \\
& \leq\left(\begin{array}{l}
u \\
v
\end{array}\right) e^{(2 \vartheta-1)(\pi / \beta)(\eta+\varsigma)}
\end{aligned}
$$

as long as $\Theta$ is large enough in order that $\sum_{l+s \geq \Theta} e^{(2 \vartheta-1)(\pi / \beta)(l+s)} \leq 1$.

From this we get item (2) since $A_{(\eta, \varsigma)}^{v, u}(n) \subset \bigcup_{j=1}^{v} A_{(\eta, \varsigma), j}^{v, u}(n)$.

For item (3) we note that $A_{(\eta, \varsigma)}(n) \subset \bigcup_{v=1}^{u} \bigcup_{u=v}^{n} A_{(\eta, \varsigma)}^{v, u}(n)$, but since there is a deep essential return at iterate $t$ (which is not an escape situation) before $n$, we know that the corresponding binding period $p$ is larger than $\iota(M)(\eta+\varsigma)>\iota(M) \Theta$.

Let us assume first that $t+p \leq n$. In this situation the maximum number $u$ of essential return situations in the first $n$ iterates of such $\omega \in \mathcal{P}_{n}$ is bounded by $n /(\iota(M) \Theta \tilde{v})$, where $\tilde{v}$ is the number of deep essential returns among the $u$ essential return situations. Since we know that $\tilde{v} \geq 1$ we get $u \leq(\iota(M) \Theta)^{-1} m$. This alone enables us to bound the measure of the subset $A_{(\eta, \varsigma)}^{*}(n)$ of $A_{(\eta, \varsigma)}(n)$ of those intervals $\omega \in \mathcal{P}_{n}$ such that we can find $t$ with $t+p \leq n$ as follows:

$$
\begin{aligned}
\lambda\left(A_{(\eta, \varsigma)}^{*}(n)\right) & \leq \sum_{v=1}^{u} \sum_{u=v}^{n} \lambda\left(A_{(\eta, \varsigma)}^{v, u}(n)\right) \\
& \leq \sum_{v=1}^{n /(\iota(M) \Theta)} \sum_{u=v}^{n} v\left(\begin{array}{l}
u \\
v
\end{array}\right) e^{(2 \vartheta-1)(\pi / \beta)(\eta+\varsigma)} \\
& =e^{(2 \vartheta-1)(\pi / \beta)(\eta+\varsigma)} \sum_{v=1}^{(\iota(M) \Theta)^{-1} n} v \sum_{u=v}^{n}\left(\begin{array}{l}
u \\
v
\end{array}\right) \\
& <e^{(2 \vartheta-1)(\pi / \beta)(\eta+\varsigma)} n \sum_{v=1}^{(\iota(M) \Theta)^{-1} n} v\left(\begin{array}{l}
n \\
v
\end{array}\right) \\
& <n e^{(2 \vartheta-1)(\pi / \beta)(\eta+\varsigma)}\left(\begin{array}{c}
\iota(M) \Theta)^{-1} n \\
(\iota(M) \Theta)^{-1} n
\end{array}\right) \sum_{v=1}^{\left(\sum^{(2)} v\right.}
\end{aligned}
$$




$$
\begin{aligned}
& <\frac{n^{3}}{(\iota(M) \Theta)^{2}} e^{(2 \vartheta-1)(\pi / \beta)(\eta+\varsigma)} e^{o(\Theta) n} \\
& \leq n^{3} e^{o(\Theta) n} e^{(2 \vartheta-1)(\pi / \beta)(\eta+\varsigma)}
\end{aligned}
$$

where we have used the bound $\left(\begin{array}{c}n \\ (\iota(M) \Theta)^{-1} n\end{array}\right) \leq e^{o(\Theta) n}$ which can be obtained by a straightforward application of Stirling's formula, as long as $\Theta$ is large enough.

Let us now assume that the only deep essential return $t$ of $\omega$ satisfies $m=t+p>n$. By definition $A_{(\eta, \varsigma)}(n) \backslash A_{(\eta, \varsigma)}^{*}(n) \subseteq A_{(\eta, \varsigma)}^{*}(m)$ and so

$$
\lambda\left(A_{(\eta, \varsigma)}(n)\right) \leq \lambda\left(A_{(\eta, \varsigma)}^{*}(n)\right)+\lambda\left(A_{(\eta, \varsigma)}^{*}(m)\right)
$$

Hence we can apply the previous reasoning with $m$ in place of $n$ to obtain

$$
\lambda\left(A_{(\eta, \varsigma)}^{*}(m)\right) \leq m^{3} e^{o(\Theta) m} e^{(2 \vartheta-1)(\pi / \beta)(\eta+\varsigma)} .
$$

Finally, since $m-n<p \leq \frac{2 \pi}{\beta \log \sigma}(\eta+\varsigma$ ) (by Lemma 4.6(a)), for $\vartheta$ small enough we have

$$
\begin{aligned}
\lambda\left(A_{(\eta, \varsigma)}^{*}\right. & (m)) \\
& \leq n^{3} e^{o(\Theta) n} e^{(2 \vartheta-1)(\pi / \beta)(\eta+\varsigma)} e^{o(\Theta)(m-n)}\left(\frac{m}{n}\right)^{3} \\
& \leq n^{3} e^{o(\Theta) n} e^{(2 o(\Theta) / \log \sigma+2 \vartheta-1)(\pi / \beta)(\eta+\varsigma)}\left(1+\frac{2 \pi(\eta+\varsigma)}{n \beta \log \sigma}\right)^{3} \\
& \leq n^{3} e^{o(\Theta) n} e^{(3 \vartheta-1)(\pi / \beta)(\eta+\varsigma)} .
\end{aligned}
$$

Putting (5.8)-(5.10) together, we complete the proof of the lemma.

6. Slow recurrence to the critical set. Now we make use of the lemmas from Section 5 to prove Theorem B and consequently also Theorem A. We start by recalling the definition of $\mathcal{C}_{n}^{b}(\omega)$ from $(1.7)$ and that $u_{n}(\omega)$ is the number of essential return situations or escape times of the $f$-orbit of $\omega \in \mathcal{P}_{n}$ between 0 and $n$.

We let $0 \leq t_{1}<\cdots<t_{u_{n}} \leq n$ be the essential return times or escape times of the orbit of each point of $\omega$ and write $\left(l_{1}, s_{1}, j_{1}\right), \ldots,\left(l_{u_{n}}, s_{u_{n}}, j_{u_{n}}\right)$ for the corresponding critical points and depths at each essential return situation, as in Section 5. We also recall that $d=d_{n}(\omega)$ is the number of pairs $\left(l_{i}, s_{i}\right)$ such that $l_{i}+s_{i} \geq \Theta$, where $\Theta=\Theta(b)$ is defined in (5.4).

We consider the sequence of deep return situations of $\omega$ : $1 \leq r_{1}<\cdots<$ $r_{d} \leq u_{n}$ among the sequence of return situations and then define

$$
\mathcal{D}_{n}^{b}(x)=\sum_{k=1}^{d}\left(\left|l_{r_{k}}\right|+\left|s_{r_{k}}\right|\right), \quad x \in \omega,
$$

which is constant on the elements of $\mathcal{P}_{n}$, and get the following bound. 
Proposition 6.1. There exists $B_{0}=B_{0}(\sigma, \rho, \tau)>0$ such that for every $\omega \in \mathcal{P}_{n}$ such that $d_{n} \mid \omega \geq 2$ we have $\mathcal{C}_{n}^{b}(x) \leq\left(B_{0} / n\right) \mathcal{D}_{n}^{b}(x)$ for all $x \in \omega$.

We start by proving the following.

LEMma 6.2. Let $1 \leq t_{i}<n$ be an essential return, inessential return or escape situation for $\omega \in \mathcal{P}_{n}$, with binding time $p_{i}=p\left(l_{i}, s_{i}\right)$ (which we set to zero in the case of an escape situation). Then there exists a constant $B_{1}>0$ such that for all $x \in \omega$,

$$
\mathcal{C}\left(t_{i}, t_{i}+p_{i}\right)=\sum_{k=t_{i}}^{t_{i}+p_{i}}-\log \operatorname{dist}_{b}\left(f^{k}(x), \mathcal{C}\right) \leq B_{1}\left(\left|l_{i}\right|+\left|s_{i}\right|\right) .
$$

Moreover, if $p_{i}>0$, then $\operatorname{dist}\left(f^{t_{i}+k}(x), \mathcal{C}\right)>\operatorname{dist}\left(f^{t_{i}}(x), \mathcal{C}\right)$ for $k=1, \ldots, p$ as long as $\rho>0$ is small enough. In particular, $\mathcal{C}\left(t_{i}, t_{i}+p_{i}\right)=0$ for escape situations or return situations which are not deep, i.e. the host interval $I(l, s, j)$ is such that $|l|+|s| \leq \Theta$.

Proof. Let us fix $x \in \omega$ in what follows. We consider first the case of $t_{i}$ being an essential return or escape situation with $\left|l_{i}\right|+\left|s_{i}\right| \leq \Theta$, i.e. $t_{i} \neq t_{r_{k}}$ for all $k=1, \ldots, d$. Thus $\log \operatorname{dist}_{b}\left(f^{t_{i}}(x), \mathcal{C}\right)=0$. If $t_{i}$ is an escape situation there is nothing else to prove because $p_{i}=0$. Otherwise $p_{i}>0$ and we have two possibilities at the binding times $t_{i}+k$ with $k=1, \ldots, p_{i}$ : either $\left|f^{t_{i}+k}(x)\right|>\varepsilon$, in which case we have again $\log \operatorname{dist}_{b}\left(f^{t_{i}+k}(x), \mathcal{C}\right)=0$; or else $\left|f^{t_{i}+k}(x)\right| \leq \varepsilon$. In this last case by Proposition 3.4 and the definition of binding time, if $\operatorname{dist}\left(f^{t_{i}+k}(x), \mathcal{C}\right) \leq \operatorname{dist}\left(f^{t_{i}}(x), \mathcal{C}\right)$, then

$$
\rho_{0} e^{-\rho k} \leq \operatorname{dist}\left(f^{t_{i}+k}(x), \mathcal{C}\right) \leq \operatorname{dist}\left(f^{t_{i}}(x), \mathcal{C}\right) \leq a_{2} e^{-(\pi / \beta)\left(\left|l_{i}\right|+\left|s_{i}\right|\right)}
$$

and thus

$$
\frac{2 \pi}{\beta \log \sigma}\left(\left|l_{i}\right|+\left|s_{i}\right|\right) \geq p_{i} \geq k \geq \frac{1}{\rho} \log \frac{\rho_{0}}{2}+\frac{\pi}{\beta \rho}\left(\left|l_{i}\right|+\left|s_{i}\right|\right),
$$

which is impossible as long as $\pi /(\beta \rho)>2 \pi /(\beta \log \sigma)$. Therefore choosing $k_{0}$ large enough and $\rho$ sufficiently small we get $\operatorname{dist}\left(f^{t_{i}+k}(x), \mathcal{C}\right)>$ $\operatorname{dist}\left(f^{t_{i}}(x), \mathcal{C}\right)$ for all $k=1, \ldots, p_{i}$ and so $\mathcal{C}\left(t_{i}, t_{i}+p_{i}\right)=0$ for escapes and returns which are not deep. This proves the last part of the statement of the lemma.

On the other hand, if $t_{i}=t_{r_{k}}$ for some $1 \leq k \leq d$, we get

$$
\operatorname{dist}_{b}\left(f^{t_{r_{k}}}(x), \mathcal{C}\right) \geq a_{2} e^{-(\pi / \beta)\left(\left|l_{r_{k}}\right|+\left|s_{r_{k}}\right|\right)}
$$

and so if $t_{r_{k}}$ is an escape or a return we have the contribution

$$
-\log \operatorname{dist}_{b}\left(f^{t_{r_{k}}}(x), \mathcal{C}\right) \leq-\log a_{2}+\frac{\pi}{\beta}\left(\left|l_{r_{k}}\right|+\left|s_{r_{k}}\right|\right)
$$

to the sum $\mathcal{C}_{n}^{b}(x)$. For escape situations the proof ends here. 
However, for return times we must consider the next binding period. We stress that we are assuming (4.1) holds.

Now for $h=1, \ldots, p_{r_{k}}, l=l_{r_{k}}$ and $i=r_{k}$ we have

$$
\operatorname{dist}\left(f^{t_{i}+h}(x), \mathcal{C}\right) \geq \operatorname{dist}\left(f^{h}\left(x_{l}\right), \mathcal{C}\right)-\operatorname{dist}\left(f^{h}\left(x_{l}\right), f^{t_{i}+h}(x)\right) .
$$

Now we consider the two cases in the relations (3.3).

CASE $\left|f^{h}\left(x_{l}\right)\right| \leq \varepsilon$. Then (6.2) is bounded from below by

$$
\left(1-e^{-\tau h}\right) \operatorname{dist}\left(f^{h}\left(x_{l}\right), \mathcal{C}\right) \text {. }
$$

CAse $\left|f^{h}\left(x_{l}\right)\right|>\varepsilon$. Then from (1.3) we have

$$
\varepsilon^{\tau}<\frac{1-e^{-\pi / \beta}}{2} \quad \text { so } \quad \varepsilon^{1+\tau}<\varepsilon \frac{1+e^{-\pi / \beta}}{2}=\varepsilon-x_{k_{0}}<\operatorname{dist}\left(f^{h}\left(x_{l}\right), \mathcal{C}\right)
$$

this ensures that (6.2) is bounded from below by the same expression (6.3) above.

Hence we deduce

$$
\sum_{h=1}^{p_{i}}-\log \operatorname{dist}\left(f^{t_{i}+h}(x), \mathcal{C}\right) \leq C(\tau)+\sum_{h=1}^{p_{i}}-\log \operatorname{dist}\left(f^{h}\left(x_{l}\right), \mathcal{C}\right) .
$$

To bound the last sum we use the assumption (4.1) on free times of the orbit of $x_{l}$. We need to sum up to the first free time of the orbit of $x_{l}$ after $p_{i}$. But if $p_{i}$ is a bound time for the orbit of $x_{l}$, then by [PRV, Lemma 5.3(a)] its binding period must be smaller than $(2 \rho / \log \sigma) p_{i}$. Thus there exists a free time $n$ for the orbit of $x_{l}$ with $p_{i} \leq n \leq(1+2 \rho / \log \sigma) p_{i}$. Hence

$$
\sum_{h=1}^{p_{i}}-\log \operatorname{dist}\left(f^{h}\left(x_{l}\right), \mathcal{C}\right) \leq \hat{M}(1+2 \rho / \log \sigma) p_{i}
$$

and so (6.4) is bounded by $C(\tau)+\hat{M}(1+2 \rho / \log \sigma) p_{i}$.

For $\varepsilon$ small enough, $k_{0}$ and $p_{i}$ are very large and in both cases (6.2) we get $\mathcal{C}\left(t_{r_{k}}, t_{r_{k}}+p_{r_{k}}\right) \leq \tilde{M} p_{r_{k}}$ for a constant $\tilde{M}$. By Lemma $4.6($ a) we obtain

$$
\mathcal{C}\left(t_{r_{k}}, t_{r_{k}}+p_{r_{k}}\right) \leq B_{1}\left(\left|l_{r_{k}}\right|+\left|s_{r_{k}}\right|\right)
$$

for a constant $B_{1}>0$, concluding the proof of the lemma.

Next we show that the depth of an inessential return or free time is not greater than the depth of the essential return situation that precedes it.

LEMMA 6.3. Let $t_{i}$ be an essential return or an escape for $\omega \in \mathcal{P}_{n}$ with $I\left(l_{i}, s_{i}, j_{i}\right) \subset f^{t_{i}}(\omega) \subset I\left(l_{i}, s_{i}, j_{i}\right)^{+}$. Then for each consecutive inessential return $t_{i}<t_{i}(1)<\cdots<t_{i}(v)<n$ before the next essential return or escape and for each free time, i.e. for all iterates $t_{i}(k)+p_{i}(k)<j \leq t_{i}(k+1)$ for $k=0, \ldots, v-1$ with $p_{i}(k)$ the binding period of the $k$ th return, the host interval $I(l, s, j) \supset f^{j}(\omega)$ is such that $|l|+|s|<\left|l_{i}\right|+\left|s_{i}\right|$. 
Proof. By items (1) and (3) of Lemma 4.8 we have $\left|f^{j}(\omega)\right|>\left|f^{t_{i}}(\omega)\right|>$ $\left|I\left(l_{i}, s_{i}, j_{i}\right)\right|$. Thus because each $j$ is an inessential return or a free time we get

$$
a_{1} \frac{e^{-(\pi / \beta)(|l|+|s|)}}{(|l|+|s|)^{3}}>\left|f^{t_{i}(k)}(\omega)\right|>a_{1} \frac{e^{-(\pi / \beta)\left(\left|l_{i}\right|+\left|s_{i}\right|\right)}}{\left(\left|l_{i}\right|+\left|s_{i}\right|\right)^{3}} .
$$

As $z^{-3} e^{-(\pi / \beta) z}$ is decreasing for $z>0$, we conclude that $|l|+|s|<\left|l_{i}\right|+\left|s_{i}\right|$.

If we have deep returns we can use sharper bounds to obtain a relation between the logarithmic distance to the critical set along the orbit between consecutive essential returns and the depth of the first return. Note that by (3.4) on "deep" free iterates between critical points we may write

$$
\left|f^{\prime}(x)\right| \geq\left(\frac{\tau \hat{x}^{\alpha-1}}{C} e^{-(1-\alpha) \frac{\pi}{\beta}|s(\tau)|}\right) e^{(1-\alpha) \frac{\pi}{\beta}(|l|+|s|)}>e^{(1-\hat{\alpha}) \frac{\pi}{\beta}(|l|+|s|)}
$$

for some $\alpha<\hat{\alpha}<1$, since at these times we have $|s| \leq s(\tau)$ and we assume that $|l|+|s| \geq \Theta$ is so large that the expression in parenthesis in (6.5) is larger than $e^{-(\hat{\alpha}-\alpha) \Theta}$.

Lemma 6.4. Let $t_{i}$ be a deep essential return or an escape for $\omega \in \mathcal{P}_{n}$ with $I\left(l_{i}, s_{i}, j_{i}\right) \subset f^{t_{i}}(\omega) \subset I\left(l_{i}, s_{i}, j_{i}\right)^{+}$. Let $t_{i}=t_{i}(0)<t_{i}(1)<\cdots<$ $t_{i}(v)<t_{i}(v+1)=t_{i+1}$ be the consecutive inessential returns before the next essential return or escape and let $p_{i}(k)$ be the corresponding binding times for $k=0, \ldots, v+1$. Define $J=\bigcup_{k=0}^{v}\left\{t_{i}(k)+p_{i}(k)+1, \ldots, t_{i}(k+1)\right\}$, the set of free iterates and of inessential return times. Assume that every such iterate $h \in J$ is contained in a "deep" interval $I\left(l_{h}, s_{h}, j_{h}\right)$, i.e. $l_{h}+s_{h} \geq \Theta$. Then there exists a constant $B_{2}>0$ such that $\sum_{h \in J}-\log \operatorname{dist}_{b}\left(f^{h}(x), \mathcal{C}\right) \leq$ $B_{2}\left(\left|l_{i}\right|+\left|s_{i}\right|\right)$.

Proof. By the choice of $\Theta$ we have $Q>1$ in Lemma 4.8, and (6.5) also holds. Then we deduce

$$
\begin{aligned}
\left|f^{t_{i+1}}(\omega)\right| \geq & \prod_{k=0}^{v} e^{(1-3 \zeta)(\pi / \beta)\left(\left|l_{t_{i}(k)}\right|+\left|s_{t_{i}(k)}\right|\right)} \\
& \times \prod_{h=t_{i}(k)+p_{i}(k)}^{t_{i}(k+1)-1} e^{(1-\hat{\alpha})(\pi / \beta)\left(\left|l_{h}\right|+\left|s_{h}\right|\right)}\left|f^{t_{i}+p_{i}}(\omega)\right|,
\end{aligned}
$$

and since we may assume that $3 \zeta<\hat{\alpha}$, by the definition of essential returns and using Lemma 4.6(b) we get

$$
\frac{e^{-(\pi / \beta)\left(\left|l_{i+1}\right|+\left|s_{i+1}\right|\right)}}{\left(\left|l_{i+1}\right|+\left|s_{i+1}\right|\right)^{3}} \geq \exp \left(\frac{\pi}{\beta}\left((1-\hat{\alpha}) \sum_{h \in J}\left(\left|l_{h}\right|+\left|s_{h}\right|\right)\right)\right) \frac{e^{-3 \zeta(\pi / \beta)\left(\left|l_{i}\right|+\left|s_{i}\right|\right)}}{\left(\left|l_{i}\right|+\left|s_{i}\right|\right)^{3}}
$$


or equivalently

$$
\begin{aligned}
3 \log \left(\frac{\left|l_{i}\right|+\left|s_{i}\right|}{\left|l_{i+1}\right|+\left|s_{i+1}\right|}\right)+3 \zeta \frac{\pi}{\beta}\left(\left|l_{i}\right|+\left|s_{i}\right|\right) & \\
& \geq \frac{\pi}{\beta}\left(\left|l_{i+1}\right|+\left|s_{i+1}\right|\right)+\frac{\pi}{\beta}\left((1-\hat{\alpha}) \sum_{h \in J}\left(\left|l_{h}\right|+\left|s_{h}\right|\right)\right) .
\end{aligned}
$$

Since $\left|l_{i+1}\right|+\left|s_{i+1}\right| \gg 1$, the left hand side of (6.6) is smaller than

$$
\begin{aligned}
\left(3 \zeta+\frac{3 \beta \log \left(\left|l_{i}\right|+\left|s_{i}\right|\right)}{\pi\left(\left|l_{i}\right|+\left|s_{i}\right|\right)}\right) \frac{\pi}{\beta}\left(\left|l_{i}\right|+\left|s_{i}\right|\right) & \leq\left(3 \zeta+\frac{3 \beta \log \Theta}{\pi \Theta}\right) \frac{\pi}{\beta}\left(\left|l_{i}\right|+\left|s_{i}\right|\right) \\
& \leq 5 \zeta \frac{\pi}{\beta}\left(\left|l_{i}\right|+\left|s_{i}\right|\right)
\end{aligned}
$$

for $\Theta$ sufficiently large, because $\left|l_{i}\right|+\left|s_{i}\right| \geq \Theta$ and $\log (z) / z$ is decreasing for $z>e$. Thus we get

$$
5 \zeta\left(\left|l_{i}\right|+\left|s_{i}\right|\right) \geq\left|l_{i+1}\right|+\left|s_{i+1}\right|+(1-\hat{\alpha}) \sum_{h \in J}\left(\left|l_{h}\right|+\left|s_{h}\right|\right) .
$$

Now since every iterate is "deep", for all $x \in \omega$ we have the bound

$\sum_{h \in J}-\log \operatorname{dist}_{b}\left(f^{h}(x), \mathcal{C}\right) \leq-\# J \cdot \log a_{2}+\frac{\pi}{\beta} \sum_{h \in J}\left(\left|l_{h}\right|+\left|s_{h}\right|\right) \leq B_{2}\left(\left|l_{i}\right|+\left|s_{i}\right|\right)$

for a constant $B_{2}>0$ depending on $\zeta$ and $\hat{\alpha}$ from (6.7), as long as $\left|l_{h}\right|+\left|s_{h}\right|$ $\geq \Theta$ is sufficiently large. This completes the proof of the lemma.

Proof of Proposition 6.1. Let us fix $x \in \omega \in \mathcal{P}_{n}$ with $d_{n} \mid \omega \geq 2$ and $i \in\left\{1, \ldots, u_{n}(x)-1\right\}$. According to Remark 2.1 and the definition of deep essential return situation we have

$$
\operatorname{dist}_{b}\left(f^{r_{i}}(x), \mathcal{C}\right) \geq a_{2} e^{-(\pi / \beta)\left(\left|l_{i}\right|+\left|s_{i}\right|\right)} .
$$

Note that the above truncated distance is 1 on the return situations $t_{i}$ which are not deep, by the choice of $\Theta$. Moreover, this distance is also 1 for all iterates between such $t_{i}$ (not deep) and the next return situation $t_{i+1}$ by Lemma 6.3 for the inessential return and free iterates, and by Lemma 6.2 for the bound iterates.

Hence we only have to take care of the deep essential return or escape times plus the next iterates before the following essential return situation. The sum of the logarithms of the truncated distance on binding periods, given by Lemma 6.2 , is bounded by a constant times the depth of the return which originated the binding. In addition, the same sum over the free and the inessential return iterates is likewise bounded by the depth of the essential return or escape $t_{i}$, by Lemma 6.4. If we keep the notations introduced in 
the statements of Lemmas 6.3 and 6.4 , then we may write

$$
\begin{aligned}
\mathcal{C}\left(t_{i}, t_{i+1}\right) & \leq \sum_{k=0}^{v}\left[\mathcal{C}\left(t_{i}(k), t_{i}(k)+p_{i}(k)\right)+\mathcal{C}\left(t_{i}(k)+p_{i}(k)+1, t_{i}(k+1)\right)\right] \\
& \leq B_{1} \sum_{k=0}^{v}\left(\left|l_{t_{i}(k)}\right|+\left|s_{t_{i}(k)}\right|\right)+B_{2}\left(\left|l_{i}\right|+\left|s_{i}\right|\right) \\
& \leq B_{1} \sum_{k=0}^{v} \mathcal{C}\left(t_{i}(k)+p_{i}(k)+1, t_{i}(k+1)\right)+B_{2}\left(\left|l_{i}\right|+\left|s_{i}\right|\right) \\
& \leq B_{2}\left(1+B_{1}\right)\left(\left|l_{i}\right|+\left|s_{i}\right|\right)
\end{aligned}
$$

Setting $B_{0}=B_{2}\left(1+B_{1}\right)$ finishes the proof of Proposition 6.1.

6.1. The expected value of the distance at return times. Proposition 6.1 together with Lemma 5.1 and Proposition 5.2 ensure that, to obtain slow recurrence to the critical set, we need to bound $\mathcal{D}_{n}^{b}(x) / n$ for Lebesgue almost every $x \in I$. Indeed, for every large enough $n$ we have

$$
\begin{aligned}
\left\{x \in I: \mathcal{C}_{n}^{b}(x)>\delta\right\} \subseteq \bigcup\left\{\omega \in \mathcal{P}_{n}: u_{n} \mid \omega \equiv 1\right\} \\
\\
\qquad\left\{x \in I: u_{n}(x) \geq 2 \text { and } \mathcal{D}_{n}^{b}(x)>n \delta / B_{0}\right\},
\end{aligned}
$$

and Lemma 5.1 shows that the left hand side subset of the above union has exponentially small measure. We now show that Proposition 5.2 implies a similar bound for the right hand subset.

Lemma 6.5. For all $z \in(0,(1-2 \vartheta) \pi /(2 \beta))$ there is $\Theta_{1}$ so that

$$
\int e^{z \mathcal{D}_{n}^{b}(x)} d \lambda(x) \leq e^{o(\Theta) n}
$$

for $\Theta>\Theta_{1}=\Theta_{1}(z, \tau, \rho, \sigma)$, where $o(\Theta) \rightarrow 0$ as $\Theta \rightarrow \infty$.

Proof. The integral in the statement equals the following series:

$$
\sum_{\substack{1 \leq v \leq u \leq n \\\left(\eta_{1}, v_{1}\right), \ldots,\left(\eta_{v}, v_{v}\right)}} \exp \left(z \sum_{k=1}^{v}\left(\eta_{k}+v_{k}\right)\right) \cdot \lambda\left(A_{\left(\eta_{1}, v_{1}\right), \ldots,\left(\eta_{v}, v_{v}\right)}^{u, v}(n)\right),
$$

where $\eta_{k}+v_{k} \geq \Theta, v_{k} \geq 1$ and $\eta_{k} \geq k_{0}$ for $k=1, \ldots, v$. Proposition 5.2 provides the bound

$$
\begin{array}{r}
\sum_{1 \leq v \leq u \leq n,\left(\eta_{1}, v_{1}\right), \ldots,\left(\eta_{v}, v_{v}\right)}\left(\begin{array}{l}
u \\
v
\end{array}\right) e^{z \sum_{k=1}^{v}\left(\eta_{k}+v_{k}\right)+(2 \vartheta-1)(\pi / \beta) \sum_{k=1}^{v}\left(\eta_{k}+v_{k}\right)} \\
=\sum_{1 \leq v \leq u \leq n,\left(\eta_{1}, v_{1}\right), \ldots,\left(\eta_{v}, v_{v}\right)}\left(\begin{array}{l}
u \\
v
\end{array}\right) e^{(z+(2 \vartheta-1) \pi / \beta) \sum_{k=1}^{v}\left(\eta_{k}+v_{k}\right)} .
\end{array}
$$


Now setting $\Delta=\sum_{k=1}^{v}\left(\eta_{k}+v_{k}\right)$ and

$$
\begin{aligned}
K(v, \Delta)=\#\left\{\left(\left(l_{1}, s_{1}\right), \ldots,\left(l_{v}, s_{v}\right)\right)\right. & : \sum_{k=1}^{v}\left(l_{k}+s_{k}\right)=\Delta, \\
& \left.l_{k} \geq k_{0}, s_{k} \geq 1, l_{k}+s_{k} \geq \Theta, 1 \leq k \leq v\right\}
\end{aligned}
$$

we may rewrite the last series as

$$
\sum_{1 \leq v \leq u \leq n} \sum_{\Delta \geq v \Theta}\left(\begin{array}{l}
u \\
v
\end{array}\right) K(v, \Delta) e^{(z+(2 \vartheta-1) \pi / \beta) \Delta} .
$$

To estimate $K(v, \Delta)$ we observe that

$$
\begin{aligned}
K(v, \Delta) & \leq \#\left\{\left(n_{1}, \ldots, n_{2 v}\right): \sum_{k=1}^{2 v} n_{k}=\Delta \text { and } n_{k} \geq 0, k=1, \ldots, 2 v\right\} \\
& =\left(\begin{array}{c}
\Delta+2 v-1 \\
2 v-1
\end{array}\right) .
\end{aligned}
$$

By a standard application of Stirling's formula we get

$$
K(v, \Delta) \leq\left(C^{1 / \Delta}\left(1+\frac{2 v-1}{\Delta}\right)\left(1+\frac{\Delta}{2 v-1}\right)^{(2 v-1) / \Delta}\right)^{\Delta} \leq e^{z \Delta},
$$

since $\Delta \geq v \Theta$ ensures that the expression in parentheses can be made arbitrarily close to 1 if $\Theta$ is taken greater than some constant $\Theta_{0}=\Theta_{0}(z)$, where $0<C<1$ is a constant independent of $\Theta$ and we assume that $z>0$ is small. Hence we arrive at

$$
\begin{aligned}
\int e^{z \mathcal{D}_{n}^{b}(x)} d \lambda(x) & \leq \sum_{1 \leq v \leq u \leq n} \sum_{\Delta \geq v \Theta}\left(\begin{array}{l}
u \\
v
\end{array}\right) e^{(2 z+(2 \vartheta-1) \pi / \beta) \Delta} \\
& \leq \sum_{v=0}^{n}\left(\begin{array}{l}
n \\
v
\end{array}\right) C e^{(2 z+(2 \vartheta-1) \pi / \beta) \Theta v} \\
& \leq\left(1+C e^{(2 z+(2 \vartheta-1) \pi / \beta) \Theta}\right)^{n}=e^{o(\Theta) n}
\end{aligned}
$$

as long as $0<z<(1-2 \vartheta) \pi /(2 \beta)$ and $\Theta>\Theta_{1}>\max \left\{\theta, \Theta_{0}\right\}$ is large enough so that $Q>1$ in Lemma 4.8 and (6.5) holds, as stated.

As a consequence of this bound we can use Chebyshev's inequality with $z$ and $\Theta$ as in the statement of Lemma 6.5 to obtain

$$
\begin{aligned}
\lambda\left(\left\{\mathcal{D}_{n}^{b} \geq n \delta / B_{0}\right\}\right) & =\lambda\left(\left\{e^{z \mathcal{D}_{n}^{b}}>e^{z \delta n / B_{0}}\right\}\right) \leq e^{-z \delta n / B_{0}} \int e^{z \mathcal{D}_{n}^{b}} d \lambda \\
& \leq e^{-z \delta n / B_{0}} e^{o(\Theta) n} .
\end{aligned}
$$

Now observe that we may take $b=b(\delta)>0$ so small that $\Theta(b)$ becomes large enough to satisfy all constraints and moreover $o(\Theta)<\delta z / n$. 
As already explained, this together with Lemma 5.1 implies that there are $C, \xi>0$, where $\xi=\xi(\delta)$, such that $\lambda\left(\left\{x \in I: \mathcal{C}_{n}(x)>\delta\right\}\right) \leq C e^{-\xi n}$ for all $n \geq 1$. Then since

$$
\{x \in I: \mathcal{R}(x)>n\} \subseteq \bigcup_{k>n}\left\{x \in I: \mathcal{C}_{k}(x)>\delta\right\}
$$

we conclude that there are constants $C_{1}, \xi_{1}>0$ such that the conclusion of Theorem B holds.

7. Fast expansion for most points. Here we use the results from the previous sections to prove Theorem $\mathrm{C}$ and as a consequence obtain Corollary D. We start by setting $E_{n}=\left\{\omega \in \mathcal{P}_{n}: \exists 1 \leq k \leq n\right.$ such that $\operatorname{dist}\left(f^{k}(\omega), \mathcal{C}\right)<e^{-\rho n}$,

$$
\left.f^{k}(\omega) \subset I(l, s) \text { and }|l| \geq k_{0},|s|>s(\tau)\right\}
$$

and proving the following bound.

Lemma 7.1. There are constants $C, \xi>0$ depending on $\hat{f}, k_{0}, \zeta, \rho$, and $\tau$ only such that $\lambda\left(\bigcup E_{n}\right) \leq C e^{-\xi n}$ for all $n \geq 1$.

Proof. Let us take $\omega \in E_{n}$ and let $k \in\{1, \ldots, n\}$ be the iterate which is very close to the critical set. Observe that by Remark 2.1 the constraint on the distance implies $-\rho n>\log \operatorname{dist}\left(f^{k}(\omega), \mathcal{C}\right) \geq \log a_{2}-\frac{\pi}{\beta}(|l|+|s|) \quad$ and so $\quad|l|+|s| \geq \frac{\beta \rho}{20 \pi} n$.

Since this iterate is in the binding region, there must be an essential return $t<k, t \in R_{n}(\omega)$, whose depth is at least as large as $|l|+|s|$, by the results of Lemmas 6.2 and 6.3 .

Hence, according to the definition of $A_{(\eta, v)}(n)$ from Section 5 , if $n \geq \Theta$ then

$$
\bigcup E_{n} \subset \bigcup\left\{A_{(\eta, v)}(n):(\eta, v) \text { is such that } \eta+v \geq \frac{\beta \rho}{20 \pi} n\right\} .
$$

Thus by Corollary 5.3 we can estimate

$$
\begin{aligned}
\lambda\left(\bigcup E_{n}\right) & \leq n^{3} e^{o(\Theta) n} \sum_{\substack{\eta+v \geq \beta \rho n /(20 \pi) \\
\eta \geq k_{0}, v \geq s(\tau)}} e^{(4 \vartheta-1)(\pi / \beta)(\eta+v)} \\
& \leq n^{3} e^{o(\Theta) n} \sum_{\substack{\Delta \geq \beta \rho n /(20 \pi)\\
}} \Delta e^{(4 \vartheta-1)(\pi / \beta) \Delta} \\
& \leq C n^{3} e^{o(\Theta) n} e^{(4 \vartheta-1) \rho n / 20} \leq C e^{(4 \vartheta-1)(\rho n / 100)}
\end{aligned}
$$

for some constant $C>0$ with $\xi=(4 \vartheta-1) \rho / 100$ for a large enough $\Theta$. This finishes the proof of the lemma. 
Lemma 7.2. If $n$ is large enough, $\rho$ small enough (depending only on $\sigma$ and $A$ from Lemma 4.4) and $x \in I \backslash \bigcup E_{n}$, then $\left|\left(f^{n}\right)^{\prime}(x)\right| \geq \sigma^{n / 3}$.

Proof. Let us take $x \in \omega \in \mathcal{P}_{n} \backslash E_{n}$ and let $0<r_{1}<\cdots<r_{k}<n$ be the consecutive returns (either essential or inessential) of the first $n$ iterates of the orbit of $\omega$, and $p_{1}, \ldots, p_{k}$ the respective binding periods. We also set $q_{i}=r_{i+1}-\left(r_{i}+p_{i}+1\right)$, the free periods and possibly escape times between consecutive returns, for $i=1, \ldots, k-1, q_{0}=r_{1}$ and $q_{k}=n-\left(r_{k}+p_{k}+1\right)$ if $n>r_{k}+p_{k}$ or $q_{k+1}=0$ otherwise.

We split the argument into the following two cases. If $n>r_{k}+p_{k}$ then

$$
\begin{aligned}
\left|\left(f^{n}\right)^{\prime}(x)\right| & =\prod_{i=0}^{k}\left(\left|\left(f^{q_{i}}\right)^{\prime}\left(f^{r_{i}+p_{i}+1}(x)\right)\right| \cdot\left|\left(f^{p_{i}+1}\right)^{\prime}\left(f^{r_{i}}(x)\right)\right|\right) \\
& \geq \sigma_{0}^{\sum_{i=0}^{k+1} q_{i}} A_{0}^{k} \sigma^{\sum_{i=1}^{k}\left(p_{i}+1\right) / 3} \geq \sigma^{n / 3},
\end{aligned}
$$

since $\sigma_{0}>\tilde{\sigma}>\sigma$ and $A_{0}>1$ by Lemma 4.6(c), and also by (3.4) we may assume that at escape times the expansion rate is at least $\sigma$.

On the other hand, if $n \leq r_{k}+p_{k}$ then using Lemma 4.4, Lemma 1.3(2) and the fact that $\omega \in \mathcal{P}_{n} \backslash E_{n}$, we have

$$
\begin{aligned}
\left|\left(f^{n}\right)^{\prime}(x)\right| & =\left|\left(f^{r_{k}}\right)^{\prime}(x)\right| \cdot\left|f^{\prime}\left(f^{r_{k}}(x)\right)\right| \cdot\left|\left(f^{n-r_{k}-1}\right)^{\prime}\left(f^{r_{k}+1}(x)\right)\right| \\
& \geq\left|\left(f^{r_{k}}\right)^{\prime}(x)\right| C^{-1}\left|x_{l_{k}}\right|^{\alpha-2} e^{-\rho n} \frac{1}{A}\left|\left(f^{n-r_{k}-1}\right)^{\prime}\left(x_{l}\right)\right| \\
& \geq(C A)^{-1} \sigma^{r_{k}} e^{-\rho n\left(\frac{\alpha-2}{20}-\frac{s(\tau)}{\rho n}\right)} \sigma^{n-r_{k}-1} \\
& \geq \exp \left(n\left(\frac{n-1}{n} \log \sigma-\frac{\log (C A)}{n}-\rho\left(\frac{\alpha-2}{20}-\frac{s(\tau)}{\rho n}\right)\right) \geq \sigma^{n / 3}\right.
\end{aligned}
$$

for $\rho>0$ small enough and $n$ large enough, where $x_{l}$ is the critical point associated to $f^{r_{k}}(\omega)$ and we have also used the calculation for the previous case to estimate $\left|\left(f^{r_{k}}\right)^{\prime}(x)\right|$.

Finally, since

$$
\{x \in I: \mathcal{E}(x)>n\} \subseteq \bigcup_{k>n}\left(\bigcup\left\{\omega \in \mathcal{P}_{k}:\left|\left(f^{k}\right)^{\prime}(x)\right|<\sigma^{k / 3}, x \in \omega\right\}\right)
$$

we conclude from Lemmas 7.1 and 7.2 that there are $C_{2}, \xi_{2}>0$ such that

$$
\lambda(\{x \in I: \mathcal{E}(x)>n\}) \leq \sum_{k>n} \lambda\left(\bigcup E_{k}\right) \leq C_{2} e^{-\xi_{2} n},
$$

concluding the proof of Theorem C.

8. Exponential bound on derivatives along critical orbits. Here we explain how to obtain the bound (4.1) for the parameters in the set $S$. 
First we claim that it is enough to obtain the following bound for a sufficiently small value of $b>0$ :

$$
\mathcal{C}_{n}^{b}\left(z_{k}\right)=\sum_{j=0}^{n-1}-\log \operatorname{dist}_{b}\left(f_{\mu}^{j}\left(z_{k}\right), \mathcal{C}\right) \leq \hat{M} n,
$$

where $\hat{M}>0$ is a large constant and this bound holds for every $n \geq 1$ which is not a bound time for $z_{k}$, for every critical value $z_{k}$ with $|k| \geq k_{0}$. Indeed, fixing $n, k$ and $b>0$, if (8.1) holds then we can write

$$
\begin{aligned}
\sum_{j=0}^{n-1}-\log \operatorname{dist}\left(f_{\mu}^{j}\left(z_{k}\right), \mathcal{C}\right) \leq & \sum_{\substack{\operatorname{dist}\left(f^{j}\left(z_{k}\right), \mathcal{C}\right)<b \\
0 \leq j<n}}-\log \operatorname{dist}_{b}\left(f_{\mu}^{j}\left(z_{k}\right), \mathcal{C}\right) \\
& +\sum_{\substack{\operatorname{dist}\left(f^{j}\left(z_{k}\right), \mathcal{C}\right) \geq b \\
0 \leq j<n}}-\log b \\
& \leq \hat{M} n-\log b \cdot \#\left\{0 \leq j<n: \operatorname{dist}\left(f^{j}\left(z_{k}\right), \mathcal{C}\right) \geq b\right\} \\
\leq & (\hat{M}-\log b) n,
\end{aligned}
$$

proving the claim.

For any given $\mu \in S$ and $|k| \geq k_{0}$, let $n$ satisfy $1=r_{0}<r_{1}<\cdots<r_{u}$ $\leq n$, where $r_{1}, \ldots, r_{u}$ are the essential return situations of the orbit of $z_{k}$, in the sense of the construction performed in [PRV]. We denote by $\left(l_{i}, s_{i}\right)$ the depth corresponding to $r_{i}$ for $i=1, \ldots, u$, and set $\left(l_{0}, s_{0}\right)$ to be such that $z_{k}(\mu)=f\left(x_{k}\right) \in I\left(l_{0}, s_{0}\right)$. To prove (8.1) it suffices to obtain the following relations:

$$
\mathcal{C}_{n}^{b}\left(z_{k}\right) \leq B \sum_{i=0}^{u_{n}(k)}\left(\left|l_{i}\right|+\left|s_{i}\right|\right) \leq B n / 2,
$$

where $u_{n}(k)$ denotes the number of return situations of the orbit of $z_{k}$ up to the $n$th iterate, for every time $n \geq 1$ which is not a bound time for the orbit of $z_{k}$, for all critical values $z_{k}$ with $|k| \geq k_{0}$, and $B$ is a positive constant. Obviously both inequalities in (8.2) together imply (8.1) with $\hat{M}=B / 2$.

8.1. The left hand side from the right hand side. To obtain (8.2) we first assume that the right hand inequality has been proved for all $n \geq 1$, $|k| \geq k_{0}$ and $\mu \in S$ and deduce the left hand inequality in the same setting by induction on the number $n$ of iterates, as follows.

According to [PRV, Section 4] for a fixed $\alpha<\gamma<1$ we have

$$
\varepsilon>\left|f_{\mu}^{i}\left(z_{k}(\mu)\right)\right| \geq\left|z_{k}(\mu)\right|^{\gamma^{i-1}} \text { and }\left|\left(f_{\mu}^{i}\right)^{\prime}\left(z_{k}(\mu)\right)\right| \geq \varepsilon^{(\gamma-1) i}
$$

for every $1 \leq i<j_{0}=j_{0}(k, \mu)$, where $j_{0}$ is the first iterate of $f_{\mu}$ such that $\left|f_{\mu}^{j}\left(z_{k}(\mu)\right)\right|>\varepsilon$. This threshold $j_{0}$ is uniformly bounded from above (by $L$, 
say) for all $|k| \geq k_{0}$ and $\mu \in S$. Consequently, since all the above iterates are in the region of expansion between critical points, there are $\left|l_{i}\right| \geq k_{0}$ and $s_{i}$ such that

$$
f_{\mu}^{i}\left(z_{k}(\mu)\right) \in I\left(l_{i}, s_{i}\right) \quad \text { with } \quad\left|s_{i}\right| \leq s(\tau), i=0, \ldots, j_{0}-1 .
$$

Hence by Remark 2.1,

$$
\operatorname{dist}\left(f_{\mu}^{i}\left(z_{k}(\mu)\right), \mathcal{C}\right) \geq a_{2} e^{-(\pi / \beta)\left(\left|l_{i}\right|+\left|s_{i}\right|\right)} \geq a_{2} e^{-(\pi / \beta) s(\tau)} e^{-(\pi / \beta)\left|l_{i}\right|}
$$

and since

$$
\left|f_{\mu}^{j}\left(z_{k}(\mu)\right)\right| \leq \operatorname{dist}\left(I\left(l_{i}-1, s\right), 0\right) \leq\left(a_{2}+\hat{x}\right) e^{\pi / \beta} e^{-(\pi / \beta)\left|l_{i}\right|}
$$

we have

$$
\operatorname{dist}\left(f_{\mu}^{i}\left(z_{k}(\mu)\right), \mathcal{C}\right) \geq \frac{a_{2} e^{-(\pi / \beta) s(\tau)}}{e^{\pi / \beta}\left(a_{2}+\hat{x}\right)}\left|f_{\mu}^{i}\left(z_{k}(\mu)\right)\right| \geq K(\tau)\left|z_{k}(\mu)\right|^{\gamma^{i-1}}
$$

for $i=1, \ldots, j_{0}-1$, with $0<K(\tau)<1$. For $i=0$ we obtain

$$
\operatorname{dist}\left(z_{k}(\mu), \mathcal{C}\right) \geq a_{2} e^{-(\pi / \beta)\left(\left|l_{0}\right|+\left|s_{0}\right|\right)} \geq a_{2} e^{-(\pi / \beta) s(\tau)} e^{-(\pi / \beta)\left|l_{0}\right|} \geq K(\tau)\left|z_{k}(\mu)\right| \text {. }
$$

Because $-\log \operatorname{dist}_{b}(\cdot, \mathcal{C}) \leq-\log \operatorname{dist}(\cdot, \mathcal{C})$ we get the bound

$$
\begin{aligned}
& \sum_{i=0}^{j_{0}-1}-\log \operatorname{dist}_{b}\left(f_{\mu}^{i}\left(z_{k}(\mu)\right), \mathcal{C}\right) \\
& \quad \leq-j_{0} \log K(\tau)-\left(1+\sum_{i=1}^{j_{0}-1} \gamma^{i-1}\right) \log \left|z_{k}(\mu)\right| .
\end{aligned}
$$

Now using again Remark 2.1 we have

$$
\left|z_{k}(\mu)\right| \geq \operatorname{dist}\left(I\left(l_{0}, s_{0}\right), \mathcal{C}\right) \geq\left|\hat{x}-a_{2}\right| e^{-(\pi / \beta)\left|l_{0}\right|}>\left|\hat{x}-a_{2}\right| e^{-(\pi / \beta)\left(\left|l_{0}\right|+\left|s_{0}\right|\right)}
$$

and so (8.3) is bounded by

$$
\begin{aligned}
& -j_{0} \log K(\tau)-C \log \left|\hat{x}-a_{2}\right|+C \frac{\pi}{\beta}\left(\left|l_{0}\right|+\left|s_{0}\right|\right) \\
& \quad=\left(\left|l_{0}\right|+\left|s_{0}\right|\right)\left(C \frac{\pi}{\beta}-\frac{j_{0} \log K(\tau)+\log \left|\hat{x}-a_{2}\right|}{\left|l_{0}\right|+\left|s_{0}\right|}\right) \\
& \quad \leq\left(\left|l_{0}\right|+\left|s_{0}\right|\right)\left(C \frac{\pi}{\beta}-\frac{j_{0} \log K(\tau)+\log \left|\hat{x}-a_{2}\right|}{k_{0}+1}\right) \leq \hat{B}\left(\left|l_{0}\right|+\left|s_{0}\right|\right)
\end{aligned}
$$

for a constant $\hat{B}>0$ which depends on $\tau$. Since these initial iterates are all free, we have shown that the left hand inequality of (8.2) holds for the $j_{0}-1$ initial iterates of the orbit of every critical value for any $B \geq \hat{B}$.

Now assume that the left hand inequality of (8.2) is true for a free time $n-1$ of the orbit of a critical value $z_{k}$ for a fixed $\mu \in S$.

If $n$ is a free time for $z_{k}$ and the last essential return situation was not deep, i.e. the depth was smaller than $\Theta$, then the depth of $f^{n}\left(z_{k}\right)$ is also 
smaller than $\Theta$ by arguments akin to Lemma 6.3. Hence this situation does not contribute to the sum $\mathcal{C}_{n}^{b}\left(z_{k}\right)$.

If $n$ is a free time for $z_{k}$ and the last essential return situation was deep, then let $r \leq n-1$ be the last essential return time with depth $\left(l_{r}, s_{r}\right)$ and $t>n$ be the next return situation (either essential, inessential or escape). We claim that

$$
\sum_{j=r}^{t-1}-\log \operatorname{dist}_{b}\left(f_{\mu}^{j}\left(z_{k}(\mu)\right), \mathcal{C}\right) \leq B\left(\left|l_{r}\right|+\left|s_{r}\right|\right),
$$

which shows that the induction can be carried out up to iterate $t-1 \geq n$.

To prove the claim, note that similar arguments to those proving Lemma 6.4 show that the part of the sum (8.4) corresponding to free times after the binding period is bounded by $B_{2}\left(\left|l_{r}\right|+\left|s_{r}\right|\right)$. Hence we get (8.4) as long as $B \geq B_{2}$.

Finally, if $n$ is a return situation for $z_{k}$, either essential, inessential or an escape, we let $p$ be the binding period corresponding to the return and consider the next free iterate $n+p$ of the orbit of $z_{k}$.

If $n$ is an escape, then $p=0$ and we are done by Remark 2.1. Otherwise $0<p \leq(2 \rho / \log \sigma) n<n$ by [PRV, Lemma 5.3] and so we can use the induction hypothesis to get (6.2) with $t_{i}=n, h=1, \ldots, p, x=z_{k}$ and $x_{l}=x_{l_{n}}$, the critical point which will shadow the orbit of $z_{k}$ during the binding period. Hence we obtain the bound (6.4) as before.

Note that if $\left|l_{n}\right|+\left|s_{n}\right| \leq \Theta$, then the truncated distance is always 1 and we are done, by the same arguments as in the proof of Lemma 6.2. If we have a deep return then, analogously, we need to consider the next free time $t \geq p$ of the orbit of the bound critical value $z_{l_{n}}$ in order to properly use the induction hypothesis. We have $t \leq(1+2 \rho / \log \sigma) p$ again by [PRV, Lemma 5.3], from which we get

$$
\sum_{j=n+1}^{n+p}-\log \operatorname{dist}_{b}\left(f_{\mu}^{j}\left(z_{k}(\mu)\right), \mathcal{C}\right) \leq C(\tau)+\left(1+\frac{2 \rho}{\log \sigma}\right) p \leq B\left(\left|l_{n}\right|+\left|s_{n}\right|\right)
$$

by induction and assuming that the right hand inequality of (8.2) holds. We have also used the upper bound in Lemma 4.6(a) and assumed that

$$
B>C(\tau) / \Theta+\frac{2 \pi}{\beta \log \sigma}\left(1+\frac{2 \rho}{\log \sigma}\right) .
$$

Thus if $B$ is sufficiently large, then the inductive step can be performed in every situation.

This shows that the left hand inequality in (8.2) is true if the right hand inequality holds. 
8.2. The right hand inequality. Now we explain why we can assume that parameters $\mu \in S$ satisfy the right hand inequality of (8.2) for all critical values. The condition (30) used in [PRV, p. 463],

$$
B(n, \omega, k)=\sum_{j=1}^{n-1} p(j, \omega, k)<n / 2,
$$

to test whether a given interval $\omega$ of parameters should be excluded or not, can be replaced by

$$
\sum_{i=0}^{u_{n}(k)}\left(\left|l_{i}\right|+\left|s_{i}\right|\right) \leq n / 2
$$

without loss since $B(n, \omega, k) \leq C \sum_{i=0}^{u_{n}(k)}\left(\left|l_{i}\right|+\left|s_{i}\right|\right)$ by Claim (3) in [PRV, p. 478]. Indeed, it is this last inequality that is used in the arguments proving [PRV, Lemma 5.7] establishing the exponential bound on the measure of the set of excluded parameters. Therefore repeating the algorithm presented there step by step with the new condition (8.6) instead of (8.5) leads to the construction of a positive Lebesgue measure set $S$ satisfying Theorem 1.1 and (8.2) for all $n \geq 1$, every $|k| \geq k_{0}$ and for every $\mu \in S$. This concludes the proof of (8.1).

9. Constants depend uniformly on initial parameters. We finally complete the proof of Corollary E by explicitly showing the dependence of the constants used in the estimates of Sections 2 to 7.

In the statements of the lemmas and propositions in the aforementioned sections we stated explicitly the direct dependence of the constants appearing in each claim from earlier statements. For constants which depend only on $\hat{f}$ we used the plain letter $C$.

It is straightforward to see that every constant depends on values that ultimately rest on the choice of initial values for $\sigma, \sigma_{0}$ and $k_{0}$ and on the choice of $\rho$ and $\tau$, which are taken to be small enough and where $0<\rho<\tau$ is the unique restriction, used solely in the proof of Proposition 3.4. Note that by definition $\varepsilon=\varepsilon\left(k_{0}\right)$, and $k_{0}=k_{0}(\tau)$ according to (1.3). Thus $\tau$ can be made as small as needed.

Hence by choosing $1<\sigma<\sqrt{\tilde{\sigma}}<\sigma_{0}$ and a small $\delta$, we may then take $0<\rho<\tau$ as small as we need to obtain a small $\varepsilon>0$ (and $k_{0}$ large enough, as a consequence, see Remark 3.2), and then find $b>0$ in order that $\Theta=\Theta(b)$ be large enough so that the constants $C_{1}, C_{2}, \xi_{1}, \xi_{2}$, and consequently $C_{3}, \xi_{3}$ in the statements of Section 1 , are defined depending only on $\alpha, \beta$, which depend only on $\hat{f}$. So $C_{1}, C_{2}, C_{3}, \xi_{1}, \xi_{2}, \xi_{3}$ depend on $\sigma, \sigma_{0}, \rho$ and $\tau$, but do not depend on $\mu \in S$. This concludes the proof of Corollary E. 
Acknowledgements. We are grateful to M. J. Costa and M. Viana for helpful conversations about this work. We also thank the referees for very useful comments and the encouragement to correct a previous version of the proof.

V. A. was partially supported by CMUP-FCT (Portugal) and CNPq/ FAPERJ/Pronex Dyn. Systems (Brazil). Part of this work was done while enjoying a post-doctorate leave from CMUP at PUC-Rio and IMPA. M.J.P. was partially supported by CNPq-Brazil/Faperj-Brazil/Pronex Dyn. Systems.

\section{References}

[A] J. F. Alves, Strong statistical stability for non-uniformly expanding maps, Nonlinearity 17 (2003), 1193-1215.

[ABV] J. F. Alves, C. Bonatti and M. Viana, SRB measures for partially hyperbolic systems whose central direction is mostly expanding, Invent. Math. 140 (2000), 351-398.

[ALP] J. F. Alves, S. Luzzatto and V. Pinheiro, Lyapunov exponents and rates of mixing for one-dimensional maps, Ergodic Theory Dynam. Systems 24 (2004), 637-657.

[AOT] J. F. Alves, K. Oliveira and A. Tahzibi, On the continuity of the SRB entropy for endomorphisms, J. Statist. Phys. 123 (2006), 763-785.

[ALM] A. Avila, M. Lyubich and W. de Melo, Regular or stochastic dynamics in real analytic families of unimodal maps, Invent. Math. 154 (2003), 451-550.

[AM1] A. Avila and C. G. Moreira, Statistical properties of unimodal maps: the quadratic family, Ann. of Math. (2) 161 (2005), 831-881.

[AM2] -, 一, Statistical properties of unimodal maps: smooth families with negative Schwarzian derivative, Astérisque 286 (2003), 81-118.

[B] V. Baladi, Positive Transfer Operators and Decay of Correlations, Adv. Ser. Nonlinear Dynam. 16, World Sci., 2000.

[BP] L. Barreira and Ya. Pesin, Lectures on Lyapunov exponents and smooth ergodic theory, in: Proc. Sympos. Pure Math. 69, Amer. Math. Soc., 2001, 3-106.

[BC1] M. Benedicks and L. Carleson, On iterations of $1-a x^{2}$ on $(-1,1)$, Ann. of Math. 122 (1985), 1-25.

[BC2] -, 一, The dynamics of the Hénon map, ibid. 133 (1991), 73-169.

[BDV] C. Bonatti, L. Diaz and M. Viana, Dynamics Beyond Uniform Hyperbolicity: a Global Geometric and Probabilistic Perspective, Springer, Berlin, 2004.

[BLS] H. Bruin, S. Luzzatto and S. van Strien, Decay of correlations in one-dimensional dynamics, Ann. Sci. École Norm. Sup. 36 (2003), 621-646.

[F] J. M. Freitas, Continuity of SRB measure and entropy for Benedicks-Carleson quadratic maps, Nonlinearity 18 (2005), 831-854.

[G] S. Gouezel, Decay of correlations for nonuniformly expanding systems, Bull. Soc. Math. France 134 (2006), 1-31.

[J] M. Jakobson, Absolutely continuous invariant measures for one-parameter families of one-dimensional maps, Comm. Math. Phys. 81 (1981), 39-88.

[KH] A. Katok and B. Hasselblatt, Introduction to the Modern Theory of Dynamical Systems, Encyclopedia Math. Appl. 54, Cambridge Univ. Press, Cambridge, 1995. 
[LT] S. Luzzatto and W. Tucker, Non-uniformly expanding dynamics in maps with singularities and criticalities, Publ. Math I.H.E.S. 89 (1999), 179-226.

[LV] S. Luzzatto and M. Viana, Positive Lyapunov exponents for Lorenz-like families with criticalities, Astérisque 261 (2000), 201-237.

[M] R. Mañé, Ergodic Theory and Differentiable Dynamics, Springer, Berlin, 1987.

[MS] W. de Melo and S. van Strien, One-dimensional dynamics: the Schwarzian derivative and beyond, Bull. Amer. Math. Soc. (N.S.) 18 (1988), 159-162.

[PRV] M. J. Pacifico, A. Rovella and M. Viana, Infinite-modal maps with global chaotic behavior, Ann. of Math. 148 (1998), 441-484.

[P] Ya. Pesin, Characteristic Lyapunov exponents and smooth ergodic theory, Russian Math. Surveys 324 (1977), 55-114.

[Ro] A. Rovella, The dynamics of perturbations of the contracting Lorenz attractor, Bol. Soc. Bras. Mat. 24 (1993), 233-259.

[R] M. Rychlik, Another proof of Jakobson's theorem and related results, Ergodic Theory Dynam. Systems 8 (1988), 93-109.

[Sh] L. P. Shil'nikov, A case of the existence of a denumerable set of periodic motions, Soviet Math. Dokl. 6 (1965), 163-166.

[S] M. Shub, Global Stability of Dynamical Systems, Springer, New York, 1987.

[Sm] S. Smale, Differentiable dynamical systems, Bull. Amer. Math. Soc. 73 (1967), $747-817$.

[TTY] P. Thieullen, C. Tresser and L.-S. Young, Positive Lyapunov exponents for generic one-parameter families of unimodal maps, J. Anal. Math. 64 (1994), 121-172.

[T] M. Tsujii, Positive Lyapunov exponents in families of one-dimensional dynamical systems, Invent. Math. 111 (1993), 113-137.

[V] M. Viana, Stochastic Dynamics of Deterministic Systems, Lecture Notes XXI Braz. Math. Colloq., IMPA, Rio de Janeiro, 1997.

[Y] L.-S. Young, Statistical properties of dynamical systems with some hyperbolicity, Ann. of Math. 147 (1998), 585-650.

Vítor Araújo

Instituto de Matemática

Universidade Federal do Rio de Janeiro

C. P. 68.530

21.945-970 Rio de Janeiro, R. J., Brazil

E-mail: vitor.araujo@im.ufrj.br

and

Centro de Matemática

da Universidade do Porto

Rua do Campo Alegre 687

4169-007 Porto, Portugal

E-mail: vdaraujo@fc.up.pt
Maria José Pacifico

Instituto de Matemática

Universidade Federal do Rio de Janeiro

C. P. 68.530

21.945-970 Rio de Janeiro, R. J., Brazil

E-mail: pacifico@impa.br pacifico@im.ufrj.br http://www.dmm.im.ufrj.br

Received 4 October 2007;

in revised form 27 January 2009 